\title{
The annulation reactions of donor-acceptor cyclopropanes with (1-azidovinyl)benzene and 3-phenyl-2H-azirine
}

\author{
Joanne E. Curiel Tejeda, Lauren C. Irwin and Michael A. Kerr* \\ Department of Chemistry, the University of Western Ontario \\ London, Ontario, Canada N6A 5B7
}




\section{General information}

All reactions were carried under an Argon atmosphere unless indicated. Toluene, benzene, and dichloromethane $\left(\mathrm{CH}_{2} \mathrm{Cl}_{2}\right)$ were dried and deoxygenated by passing the nitrogen purged solvents through activated alumina columns. All other reagents and solvents were used as purchased from Sigma Aldrich, Caledon or VWR. Reaction progress was followed by thin layer chromatography (TLC) (EM Science, silica gel 60 $F_{254}$ ) visualizing with UV light, and the plates were developed using acidic anisaldehyde or $\mathrm{KMnO}_{4}$ stain. Flash chromatography was performed using silica gel purchased from Silicycle Chemical Division Inc. (230-400 mesh).

NMR experiments were performed on the Varian Mercury 400, Inova 400 and Inova 600 instruments; samples were obtained in $\mathrm{CDCl}_{3}$ (referenced to $7.26 \mathrm{ppm}$ for ${ }^{1} \mathrm{H}$ and 77.0 ppm for ${ }^{13} \mathrm{C}$ ). Coupling constants $(\mathcal{J}$ ) are in $\mathrm{Hz}$. The multiplicities of the signals are described using the following abbreviations: $s=$ singlet, $d=$ doublet, $t=$ triplet, $q=$ quartet, $\mathrm{dq}=$ doublet of quartets, $\mathrm{m}=$ multiplet, $\mathrm{b}=$ broad. Infrared spectra were obtained as thin films on $\mathrm{NaCl}$ plates using the Brucker Vector 33 FT-IR instrument. High resolution mass spectra (HRMS) were obtained on a Finnigan MAT 8200 spectrometer at $70 \mathrm{eV}$. Melting points were determined using a Gallenkamp melting point apparatus and were uncorrected.

\section{Experimental Procedure A: Synthesis of bis(2,2,2-trifluoroethyl) 2- benzylidenemalonates (2dd and 2ee).}<smiles>[R]c1cc(C=O)cc([R])c1[R]</smiles>

(1 equiv)

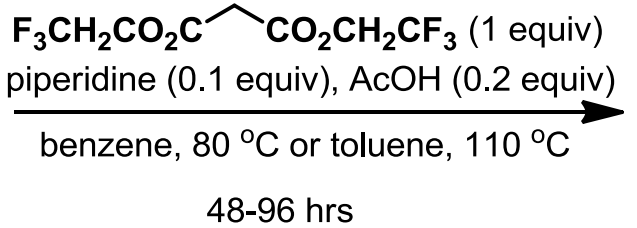

48-96 hrs

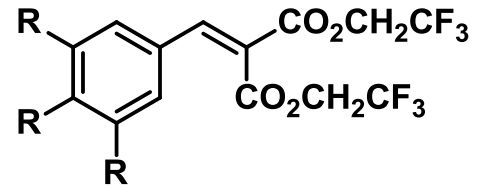

To $50-100 \mathrm{~mL}$ round-bottomed flask, the desire aldehyde (1.0 equiv), bis(2,2,2trifluoroethyl) malonate ${ }^{1}$ ( 1.0 equiv), piperidine ( 0.1 equiv), and acetic acid ( 0.2 equiv) were dissolved in benzene or toluene $(30-40 \mathrm{~mL})$. The reaction flask was fitted with a Dean-Stark apparatus and heated to $80^{\circ} \mathrm{C}$ or $110{ }^{\circ} \mathrm{C}$ for $48-96$ hours until completion (monitored by TLC). The crude mixture was washed with $5 \% \mathrm{HCl}$, saturated $\mathrm{NaHCO}_{3}$, 
and brine. The organic portion was dried with $\mathrm{MgSO}_{4}$, and filtered. The filtrate was concentrated and purified by column chromatography (EtOAc in Hexanes).

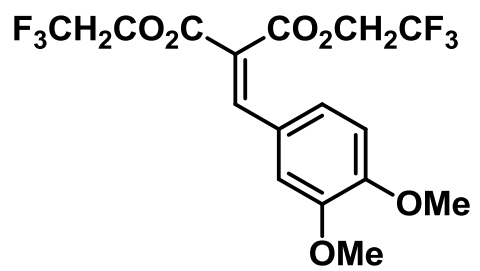

\section{Bis(2,2,2-trifluoroethyl) 2-(3,4-dimethoxybenzylidene)malonate (2dd).}

Following experimental procedure A, compound 2dd was obtained by dissolving commercially available 3,4dimethoxybenzaldehyde $(1.17 \mathrm{~g}, 7.03 \mathrm{mmol})$, bis(2,2,2-trifluoroethyl) malonate ${ }^{1}$ (1.88 $\mathrm{g}$, $7.03 \mathrm{mmol})$, piperidine $(0.07 \mathrm{~mL}, 0.703 \mathrm{mmol})$, and acetic acid $(0.08 \mathrm{~mL}, 1.41 \mathrm{mmol})$ in toluene $(30 \mathrm{~mL})$. The reaction was heated at $110^{\circ} \mathrm{C}$ for 48 hours. Compound $2 \mathbf{d d}(1.02$ $\mathrm{g}, 35 \%)$ was obtained as a white solid. MP $=82-85{ }^{\circ} \mathrm{C} . \mathrm{Rf}=0.70(40 \%$ EtOAc in hexanes).

${ }^{1} \mathrm{H}$ NMR $\left(400 \mathrm{MHz}, \mathrm{CDCl}_{3}\right) \delta=7.85(\mathrm{~s}, 1 \mathrm{H}), 7.12(\mathrm{~d}, J=8.9 \mathrm{~Hz}, 1 \mathrm{H}), 7.02(\mathrm{~d}, J=1.6 \mathrm{~Hz}$, $1 \mathrm{H}), 6.88(\mathrm{~d}, J=8.6 \mathrm{~Hz}, 1 \mathrm{H}), 4.64(\mathrm{dq}, J=14.1,8.2 \mathrm{~Hz}, 4 \mathrm{H}), 3.93(\mathrm{~s}, 3 \mathrm{H}), 3.85(\mathrm{~s}, 3 \mathrm{H})$. ${ }^{19} \mathrm{~F}$ NMR $\left(376 \mathrm{MHz}, \mathrm{CDCl}_{3}\right) \delta=-73.4$ (t, $\left.J=8.5 \mathrm{~Hz}, 3 \mathrm{~F}\right),-73.8$ (t, $\left.J=8.5 \mathrm{~Hz}, 3 \mathrm{~F}\right) .{ }^{13} \mathrm{C}$ NMR $\left(100 \mathrm{MHz}, \mathrm{CDCl}_{3}\right) \delta=164.8,162.4,152.4,149.2,146.8,125.1,121.4$ (q, J $\mathrm{J}_{\mathrm{C}-\mathrm{F}}=$ $277 \mathrm{~Hz}, 1 \mathrm{C}), 121.2$ (q, J $\left.J_{C-F}=277 \mathrm{~Hz}, 1 \mathrm{C}\right), 112.1,111.1,61.0$ (q, J $J_{C-F}=37.5 \mathrm{~Hz}, 1 \mathrm{C}$ ), 60.9 (q, $J_{C-F}=37.5 \mathrm{~Hz}, 1 \mathrm{C}$ ), 56.0, 55.8. FT-IR (thin film, $\mathrm{cm}^{-1}$ ): 3022, 2947, 2840, 1841, $1616,1514,1470,1271,1144,1084$. HRMS calc'd for $\mathrm{C}_{16} \mathrm{H}_{14} \mathrm{~F}_{6} \mathrm{O}_{6}\left[\mathrm{M}^{+}\right]$: 416.0695; found: 416.0681 .

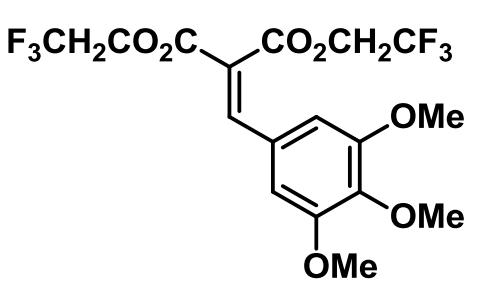

\section{Bis(2,2,2-trifluoroethyl) 2-(3,4,5-trimethoxybenzylidene)malonate (2ee).}

Following experimental procedure A, compound 2ee was obtained by dissolving commercially available 3,4,5trimethoxybenzaldehyde (1.46 g, $7.46 \mathrm{mmol})$, bis(2,2,2-trifluoroethyl) malonate ${ }^{1}(2.00 \mathrm{~g}$, $7.46 \mathrm{mmol})$, piperidine $(0.07 \mathrm{~mL}, 0.75 \mathrm{mmol})$, and acetic acid $(0.09 \mathrm{~mL}, 1.49 \mathrm{mmol})$ in benzene $(30 \mathrm{~mL})$. The reaction was heated at $80^{\circ} \mathrm{C}$ for 44 hours. Compound 2ee $(2.22$ $\mathrm{g}, 67 \%)$ was obtained as a white solid. $\mathrm{MP}=66-70{ }^{\circ} \mathrm{C} . \mathrm{Rf}=0.61(40 \%$ EtOAc in hexanes). 
${ }^{1} \mathrm{H}$ NMR $\left(400 \mathrm{MHz}, \mathrm{CDCl}_{3}\right) \delta=7.84(\mathrm{~s}, 1 \mathrm{H}), 6.74(\mathrm{~s}, 2 \mathrm{H}), 4.63(\mathrm{qd}, J=8.2,3.5 \mathrm{~Hz}, 4 \mathrm{H})$, $3.90(\mathrm{~s}, 4 \mathrm{H}), 3.85(\mathrm{~s}, 6 \mathrm{H}) .{ }^{19} \mathrm{~F}$ NMR $\left(376 \mathrm{MHz}, \mathrm{CDCl}_{3}\right) \delta=-73.5(\mathrm{t}, 8.6 \mathrm{~Hz}, 3 \mathrm{~F}),-73.9(\mathrm{t}$, $8.6 \mathrm{~Hz}, 3 \mathrm{~F}) .{ }^{13} \mathrm{C}$ NMR $\left(100 \mathrm{MHz}, \mathrm{CDCl}_{3}\right) \delta=164.5,162.1,153.4,146.8,146.8,127.0$, $121.3\left(q, J_{C-F}=277 \mathrm{~Hz}, 1 \mathrm{C}\right), 121.1\left(q, J_{C-F}=277 \mathrm{~Hz}, 1 \mathrm{C}\right), 107.4,61.0$ (q, JC-F $=37 \mathrm{~Hz}$, 1C), $60.9\left(q, J_{C-F}=37 \mathrm{~Hz}, 1 C\right)$, 56.1. FT-IR (thin film, $\mathrm{cm}^{-1}$ ): 3017, 2952, 2849, 1751, $1733,1507,1446,1280,1146,1122$. HRMS calc'd for $\mathrm{C}_{17} \mathrm{H}_{16} \mathrm{~F}_{6} \mathrm{O}_{7}\left[\mathrm{M}^{+}\right]$: 446.0800; found: 446.0808 .

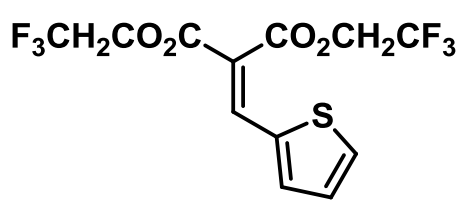

\section{Bis(2,2,2-trifluoroethyl) 2-(thiophen-2-ylmethylene)malonate (2ff).}

Following experimental procedure A, compound $\mathbf{2 f f}$ was obtained by dissolving 2-thiophenecarboxaldehyde $(1.01 \mathrm{~g}, 9.03 \mathrm{mmol})$, bis $(2,2,2-$ trifluoroethyl) malonate ${ }^{1}(2.42 \mathrm{~g}, 9.03 \mathrm{mmol})$, piperidine $(0.09 \mathrm{~mL}, 0.90 \mathrm{mmol})$, and acetic acid $(0.1 \mathrm{~mL}, 1.81 \mathrm{mmol})$ in toluene $(30 \mathrm{~mL})$. The reaction was heated at $110{ }^{\circ} \mathrm{C}$ for 48 hours. Compound $2 \mathrm{ff}(2.57 \mathrm{~g}, 78 \%)$ was obtained as a yellow oil. $\mathrm{Rf}=0.31$ (10\% EtOAc in hexanes). Product was used crude in the cyclopropanation step.

${ }^{1} \mathrm{H}$ NMR $\left(400 \mathrm{MHz}, \mathrm{CDCl}_{3}\right) \delta=8.06(\mathrm{~s}, 1 \mathrm{H}), 7.67(\mathrm{~d}, J=5.1 \mathrm{~Hz}, 1 \mathrm{H}), 7.53-7.49(\mathrm{~m}, 1 \mathrm{H})$, $7.37(\mathrm{~s}, 1 \mathrm{H}), 7.16(\mathrm{dd}, J=5.1,3.9 \mathrm{~Hz}, 1 \mathrm{H}), 4.71(\mathrm{q}, J=8.3 \mathrm{~Hz}, 2 \mathrm{H}), 4.63$ (q, $J=8.3$, $2 \mathrm{H})$.

Experimental Procedure B1: Synthesis of bis(2,2,2-trifluoroethyl)cyclopropane1,1-dicarboxylates (2d-2f).

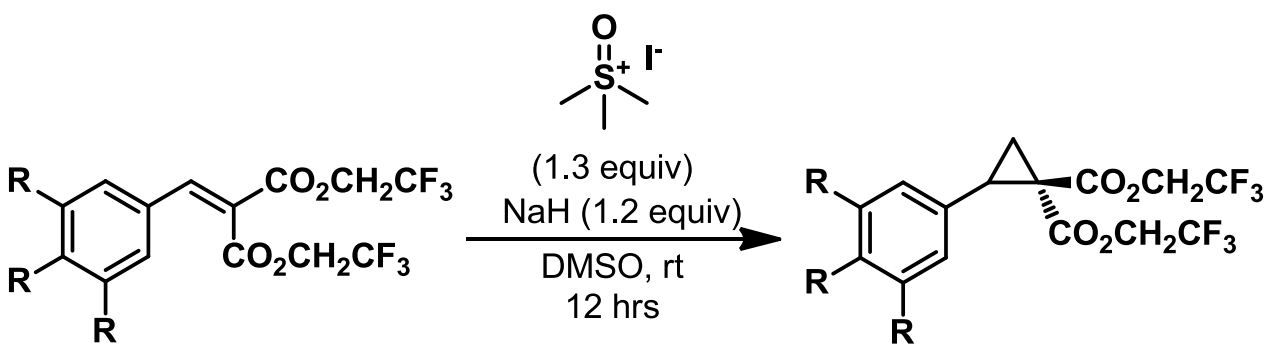

To a solution of $\mathrm{SO}\left(\mathrm{CH}_{3}\right)_{3} \mathrm{l}$ (1.3 equiv) in DMSO (10-15mL), $\mathrm{NaH}$ (1.2 equiv) was added portion wise, and stirred at room temperature for 45 minutes. The desired bis(2,2,2trifluoroethyl)2-benzylidenemalonate (1.0 equiv) was diluted in DMSO (10-15mL) and added dropwise to the reaction mixture. The reaction was stirred for 12 hours at room 
temperature. Upon completion, the mixture was diluted with $\mathrm{H}_{2} \mathrm{O}(60 \mathrm{~mL})$ and extracted with $\mathrm{Et}_{2} \mathrm{O}$. The organic portion was washed with water, brine, dried with $\mathrm{MgSO}_{4}$, and filtered. The filtrate was concentrated and purified by column chromatography (EtOAc in Hexanes).

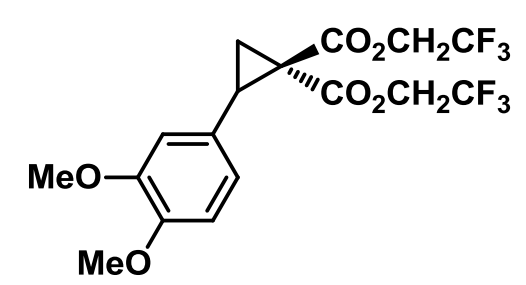

\section{Bis(2,2,2-trifluoroethyl) 2-(3,4-dimethoxyphenyl)cyclopropane- 1,1-dicarboxylate (2d).}

Following experimental procedure B1, compound 2d was prepared by dissolving $\mathrm{SO}\left(\mathrm{CH}_{3}\right)_{3} \mathrm{l}(650 \mathrm{mg}, 2.93 \mathrm{mmol})$ and $60 \%$

$\mathrm{NaH}(120 \mathrm{mg}, 2.93 \mathrm{mmol})$ in $10 \mathrm{~mL}$ of DMSO. After stirring at room temperature for 45 mins, bis(2,2,2-trifluoroethyl) 2-(3,4-dimethoxybenzylidene)malonate 2dd (1.02 g, 2.44 $\mathrm{mmol}$ ) in $10 \mathrm{~mL}$ DMSO was added dropwise. The reaction was stirred for 12 hours at room temperature. Compound $\mathbf{2 d}$ (380 $\mathrm{mg}, 36 \%)$ was obtained as white solid. MP $=98$ $-101^{\circ} \mathrm{C} . \mathrm{Rf}=0.61(40 \%$ EtOAc in hexanes).

${ }^{1} \mathrm{H}$ NMR $\left(400 \mathrm{MHz}, \mathrm{CDCl}_{3}\right) \delta=6.75(\mathrm{~m}, 3 \mathrm{H}), 4.62(\mathrm{q}, J=8.2 \mathrm{~Hz}, 1 \mathrm{H}), 4.54(\mathrm{q}, J=8.2$ $\mathrm{Hz}, 1 \mathrm{H}$ ), 4.19 (q, $J=8.2 \mathrm{~Hz}, 1 \mathrm{H}), 4.11(\mathrm{q}, J=7.8 \mathrm{~Hz}, 1 \mathrm{H}$ ), $3.85(\mathrm{~s}, 6 \mathrm{H}), 3.36(\mathrm{t}, J=9.0$ $\mathrm{Hz}, 1 \mathrm{H}), 2.33(\mathrm{dd}, J=5.5 \mathrm{~Hz}, 3.12 \mathrm{~Hz}, 1 \mathrm{H}), 1.86(\mathrm{dd}, J=5.5 \mathrm{~Hz}, 3.9 \mathrm{~Hz}, 1 \mathrm{H}) .{ }^{19} \mathrm{~F}$ NMR $\left(376 \mathrm{MHz}, \mathrm{CDCl}_{3}\right) \delta=-73.9(\mathrm{t}, J=8.6 \mathrm{~Hz}, 3 \mathrm{~F}),-74.0(\mathrm{t}, J=8.6 \mathrm{~Hz}, 3 \mathrm{~F}) .{ }^{13} \mathrm{C}$ NMR $(100$ $\left.\left.\mathrm{MHz}_{\mathrm{CDCl}}\right)\right) \delta=168.0,164.8,149.1,125.5,121.5\left(\mathrm{q}, \mathrm{J}_{\mathrm{C}-\mathrm{F}}=277 \mathrm{~Hz}, 1 \mathrm{C}\right), 121.3\left(\mathrm{q}, \mathrm{J}_{\mathrm{C}-\mathrm{F}}\right.$ $=277 \mathrm{~Hz}, 1 \mathrm{C}), 121.0,112.3,111.1,61.6\left(\mathrm{q}, J_{\mathrm{C}-\mathrm{F}}=37 \mathrm{~Hz}, 1 \mathrm{C}\right), 61.4\left(\mathrm{q}, J_{\mathrm{C}-\mathrm{F}}=37 \mathrm{~Hz}\right.$, 1C), 56.1, 36.6, 34.5, 20.4. FT-IR (thin film, $\mathrm{cm}^{-1}$ ): 3010, 2968, 2838, 1727, 1519, 1253, 1157, 1137, 1023, 968. HRMS calc'd for $\mathrm{C}_{17} \mathrm{H}_{16} \mathrm{~F}_{6} \mathrm{O}_{6}\left[\mathrm{M}^{+}\right]$: calc'd 430.0851; found: 430.0864 .

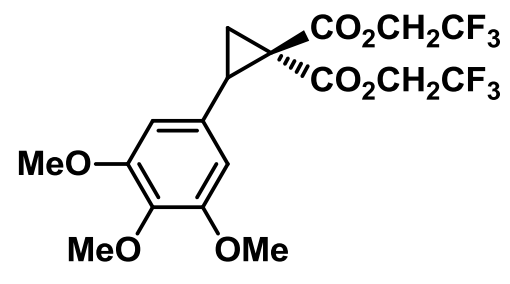

Bis(2,2,2-trifluoroethyl) 2-(3,4,5-trimethoxyphenyl)cyclopropane1,1-dicarboxylate (2e).

Following experimental procedure $\mathrm{B} 1$, compound $\mathbf{2 e}$ was prepared by dissolving $\mathrm{SO}\left(\mathrm{CH}_{3}\right)_{3} \mathrm{l}(1.29 \mathrm{~g}, 5.87 \mathrm{mmol})$, and $60 \%$ $\mathrm{NaH}(230 \mathrm{mg}, 5.87 \mathrm{mmol})$ in $15 \mathrm{~mL}$ of DMSO. After stirring at room temperature for 45 
mins, bis(2,2,2-trifluoroethyl) 2-(3,4,5-trimethoxybenzylidene)malonate 2ee (2.18 g, 4.89 $\mathrm{mmol}$ ) in $15 \mathrm{~mL}$ DMSO was added dropwise. The reaction was stirred for 12 hours at room temperature. Compound $2 \mathrm{e}(760 \mathrm{mg}, 34 \%)$ was obtained as white solid. MP $=40$ $-44{ }^{\circ} \mathrm{C} . \mathrm{Rf}=0.68$ (40\% EtOAc in Hexanes).

${ }^{1} \mathrm{H}$ NMR $\left(600 \mathrm{MHz}, \mathrm{CDCl}_{3}\right) \delta=6.41(\mathrm{~s}, 2 \mathrm{H}), 4.70-4.58(\mathrm{~m}, 2 \mathrm{H}), 4.51-4.48(\mathrm{~m}, 1 \mathrm{H}), 4.15-$ $4.21(\mathrm{~m}, 1 \mathrm{H}), 3.82(\mathrm{~s}, 6 \mathrm{H}), 3.79(\mathrm{~s}, 3 \mathrm{H}), 3.35(\mathrm{t}, J=8.8 \mathrm{~Hz}, 1 \mathrm{H}), 2.31$ (dd, J = 8.2, 5.5 $\mathrm{Hz}, 1 \mathrm{H}), 1.85(\mathrm{dd} J=9.4,5.5 \mathrm{~Hz}, 1 \mathrm{H}) .{ }^{19} \mathrm{~F} \mathrm{NMR}\left(376 \mathrm{MHz}, \mathrm{CDCl}_{3}\right) \delta=-73.9(\mathrm{t}, J=8.5$ $\mathrm{Hz}, 3 \mathrm{~F}),-74.0$ (t, $J=8.5 \mathrm{~Hz}, 3 \mathrm{~F}) .{ }^{13} \mathrm{C}$ NMR $\left(150 \mathrm{MHz}, \mathrm{CDCl}_{3}\right) \delta=167.6,164.4,153.4$, 153.1, 147.1, 128.4, 123.9 (q, J $J_{C-F}=278 \mathrm{~Hz}, 1 \mathrm{C}$ ), 123.7 (q, J $J_{C-F}=278 \mathrm{~Hz}, 1 \mathrm{C}$ ), 107.4, 105.7, $61.3\left(q, J_{C-F}=37 \mathrm{~Hz}, 1 \mathrm{C}\right), 61.1\left(q, J_{C-F}=37 \mathrm{~Hz}, 1 \mathrm{C}\right), 56.0,36.3,34.4,20.1$. FTIR (thin film, $\mathrm{cm}^{-1}$ ): 2967, 2848, 1733, 1508, 1416, 1256, 1165, 1122, 974, 658. HRMS calc'd for $\mathrm{C}_{18} \mathrm{H}_{18} \mathrm{~F}_{6} \mathrm{O}_{7}\left[\mathrm{M}^{+}\right]$: calc'd 460.0957; found: 460.0959 .

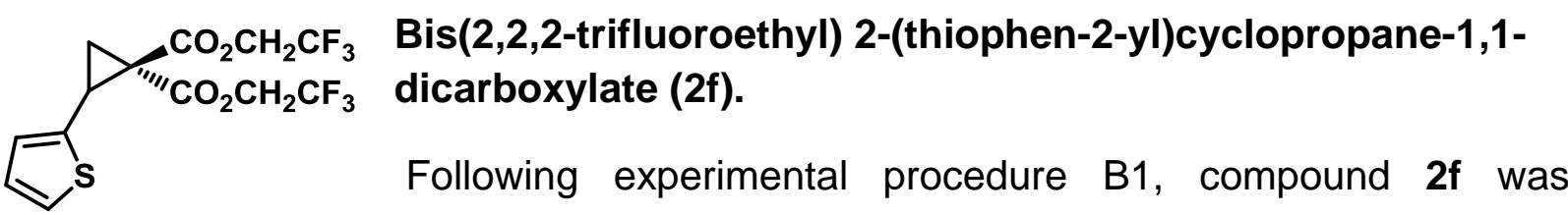
prepared by dissolving $\mathrm{SO}\left(\mathrm{CH}_{3}\right)_{3} \mathrm{l}(1.87 \mathrm{~g}, 8.51 \mathrm{mmol})$, and $60 \% \mathrm{NaH}(355 \mathrm{mg}, 8.51$ $\mathrm{mmol})$ in $15 \mathrm{~mL}$ of DMSO. After stirring at room temperature for 45 mins, bis(2,2,2trifluoroethyl) 2-(thiophen-2-ylmethylene)malonate $2 \mathrm{ff}(2.57 \mathrm{~g}, 7.09 \mathrm{mmol})$ in $15 \mathrm{~mL}$ DMSO was added dropwise. The reaction was stirred for 12 hours at room temperature. Compound $2 f(740 \mathrm{mg}, 28 \%)$ was obtained as yellow oil. $\mathrm{Rf}=0.38(10 \%$ EtOAc in Hexanes).

${ }^{1} \mathrm{H}$ NMR $\left(600 \mathrm{MHz}, \mathrm{CDCl}_{3}\right) \delta=7.20(\mathrm{~d}, J=5.1 \mathrm{~Hz}, 1 \mathrm{H}), 6.91(\mathrm{t}, J=4.7 \mathrm{~Hz}, 1 \mathrm{H}), 6.87(\mathrm{~d}$, $J=3.5 \mathrm{~Hz}, 1 \mathrm{H}), 4.59(\mathrm{q}, J=8.5 \mathrm{~Hz}, 1 \mathrm{H}), 4.53(\mathrm{q}, J=8.5 \mathrm{~Hz}, 1 \mathrm{H}), 4.31(\mathrm{q}, J=8.5 \mathrm{~Hz}$, $1 \mathrm{H}), 4.16(\mathrm{q}, J=8.5 \mathrm{~Hz}, 1 \mathrm{H}$ ), $3.44(\mathrm{t}, J=7.8 \mathrm{~Hz}, 1 \mathrm{H}), 2.31$ (dd, $J=5.15 \mathrm{~Hz}, 2.3 \mathrm{~Hz}, 1 \mathrm{H}$ ), $1.96(\mathrm{dd}, J=5.4 \mathrm{~Hz}, 3.1 \mathrm{~Hz}, 1 \mathrm{H}) .{ }^{19} \mathrm{~F}$ NMR $\left(376 \mathrm{MHz}, \mathrm{CDCl}_{3}\right) \delta=-73.9$ (t, $J=8.6 \mathrm{~Hz}$, 3F), -74.0 (t, $J=8.6 \mathrm{~Hz}, 3 \mathrm{~F}) .{ }^{13} \mathrm{C}$ NMR $\left(100 \mathrm{MHz}, \mathrm{CDCl}_{3}\right) \delta=167.2,164.1,136.2$, 126.9, 125.9, $121.1\left(\mathrm{q}, J_{\mathrm{C}-\mathrm{F}}=277 \mathrm{~Hz}, 1 \mathrm{C}\right), 121.0$ (q, JC-F $\left.=277 \mathrm{~Hz}, 1 \mathrm{C}\right), 61.2\left(\mathrm{q}, J_{\mathrm{C}-\mathrm{F}}=\right.$ $38 \mathrm{~Hz}, 1 \mathrm{C}$ ), 61.1 (q, $J_{\mathrm{C}-\mathrm{F}}=37 \mathrm{~Hz}, 1 \mathrm{C}$ ), 37.1, 28.8, 21.7. FT-IR (thin film, $\mathrm{cm}^{-1}$ ): 3113 , 
2976, 1743, 1412, 1276, 1259, 1158, 1117, 975, 703. HRMS calc'd for $\mathrm{C}_{13} \mathrm{H}_{10} \mathrm{~F}_{6} \mathrm{O}_{4} \mathrm{~S}$ $\left[\mathrm{M}^{+}\right]$: 376.0204 ; found: 376.0202 .

Experimental Procedure B2: Synthesis of bis(2,2,2-trifluoroethyl)cyclopropane1,1-dicarboxylates (2g-h and 2j-k).

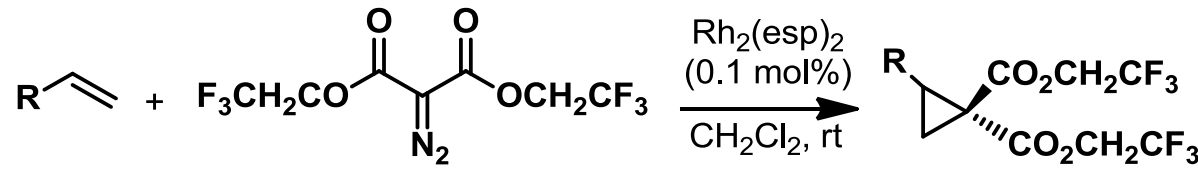

To a solution of the desired styrene (1.0 equiv), in $\mathrm{CH}_{2} \mathrm{Cl}_{2}$ (4 $\mathrm{mL}-8 \mathrm{~mL}$ ), $\mathrm{Rh}_{2}(\mathrm{esp})_{2}$ catalyst $(0.1 \mathrm{~mol} \%)$ was added. The bis(2,2,2-trifluoroethyl)2-diazomalonate ${ }^{2}$ (1.3 equivs) was dissolved in $\mathrm{CH}_{2} \mathrm{Cl}_{2}(3 \mathrm{~mL})$ and added dropwise over a period of 20 mins at room temperature. The reaction was stirred at room temperature until completion (monitored by TLC). The reaction mixture was quenched with saturated $\mathrm{NaHCO}_{3}$ and extracted with $\mathrm{CH}_{2} \mathrm{Cl}_{2}$. The organic portion was dried with $\mathrm{MgSO}_{4}$ and filtered. The filtrate was concentrated and purified by column chromatography (EtOAc in Hexanes).

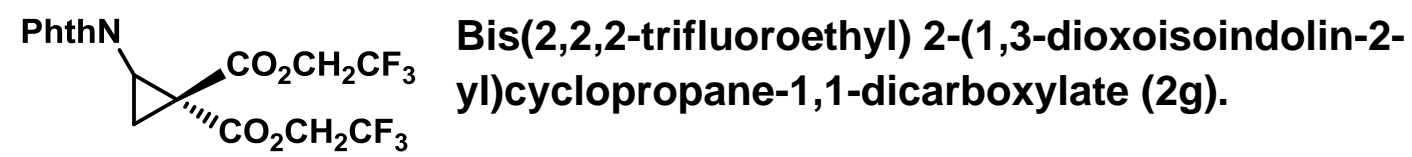

Following experimental procedure B2, cyclopropane $\mathbf{2 g}$ was prepared by dissolving commercially available $N$-vinylphthalimide $(1.00 \mathrm{~g}, 5.77 \mathrm{mmol})$ and $\mathrm{Rh}_{2}$ (esp) 2 (4.40 mg, $0.005 \mathrm{mmol}$ ) in $15 \mathrm{~mL}$ of $\mathrm{CH}_{2} \mathrm{Cl}_{2}$ followed by the addition of bis(2,2,2-trifluoroethyl)2-diazomalonate ${ }^{2}(2.21 \mathrm{~g}, 7.50 \mathrm{mmol})$ dissolved in $5 \mathrm{~mL}$ of $\mathrm{CH}_{2} \mathrm{Cl}_{2}$. Cyclopropane $2 \mathrm{~g}(1.87 \mathrm{~g}, 74 \%$ yield $)$ was obtained as a colourless oil. $\mathrm{Rf}=$ 0.57 (20\% EtOAc in hexanes).

${ }^{1} \mathrm{H}$ NMR $\left(600 \mathrm{MHz}, \mathrm{CDCl}_{3}\right) \delta=7.82(\mathrm{t}, J=3.5 \mathrm{~Hz}, 2 \mathrm{H}), 7.73(\mathrm{t}, J=2.9 \mathrm{~Hz}, 2 \mathrm{H}), 4.60$ (q, $J=8.2 \mathrm{~Hz}, 2 \mathrm{H}$ ), 4.42 (q, $J=8.2 \mathrm{~Hz}, 1 \mathrm{H}), 4.35$ (q, $J=8.2 \mathrm{~Hz}, 1 \mathrm{H}$ ), 3.81 (dd, $J=7.6 \mathrm{~Hz}$, $1 \mathrm{H}), 2.88(\mathrm{t}, J=6.5 \mathrm{~Hz}, 1 \mathrm{H}), 2.17(\mathrm{dd}, J=7.0 \mathrm{~Hz}, 1 \mathrm{H}) .{ }^{19} \mathrm{~F}$ NMR $\left(376 \mathrm{MHz}, \mathrm{CDCl}_{3}\right) \delta=-$ $73.9(\mathrm{t}, J=8.6 \mathrm{~Hz}, 3 \mathrm{~F}),-74.0(\mathrm{t}, \mathrm{J}=8.6 \mathrm{~Hz}, 3 \mathrm{~F}) .{ }^{13} \mathrm{C}$ NMR $\left(150 \mathrm{MHz}, \mathrm{CDCl}_{3}\right) \delta=167.5$, 166.2, 164.4, 134.5, 131.2, 123.6, 121.1 (q, $\left.J_{C-F}=277 \mathrm{~Hz}, 1 \mathrm{C}\right), 120.95$ (q, JC-F $=277$ $\mathrm{Hz}, 1 \mathrm{C}), 61.7\left(\mathrm{q}, J_{\mathrm{C}-\mathrm{F}}=37 \mathrm{~Hz}, 1 \mathrm{C}\right), 61.5\left(\mathrm{q}, \mathrm{J}_{\mathrm{C}-\mathrm{F}}=37 \mathrm{~Hz}, 1 \mathrm{C}\right), 36.2,32.5,20.8$. FT-IR 
(thin film, $\mathrm{cm}^{-1}$ ): $3117,3031,2985,1763,1717,1406,1259,1163,1132$, 965. HRMS calc'd for $\mathrm{C}_{17} \mathrm{H}_{11} \mathrm{~F}_{6} \mathrm{O}_{6}\left[\mathrm{M}^{+}\right]$: 439.0491 ; found: 439.0474 .

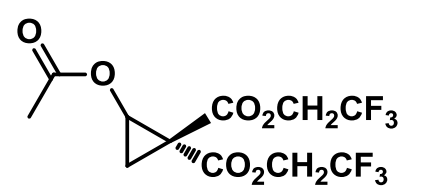

Bis(2,2,2-trifluoroethyl) 2-acetoxycyclopropane-1,1-dicarboxylate (2h).

prepared by dissolving commercially available vinyl acetate $(200 \mathrm{mg}, 2.32 \mathrm{mmol})$ and $\mathrm{Rh}_{2}$ (esp) $)_{2}(1.76 \mathrm{mg}, 0.002 \mathrm{mmol})$ in $5 \mathrm{~mL}$ of $\mathrm{CH}_{2} \mathrm{Cl}_{2}$ followed by the addition of bis(2,2,2-trifluoroethyl)2-diazomalonate ${ }^{2}(887 \mathrm{mg}, 3.02 \mathrm{mmol}$ ) dissolved in $1 \mathrm{~mL}$ of $\mathrm{CH}_{2} \mathrm{Cl}_{2}$. Cyclopropane $2 \mathrm{~h}(711 \mathrm{~g}, 87 \%$ yield $)$ was obtained as a colourless oil. $\mathrm{Rf}=0.49$ (30\% EtOAc in hexanes).

${ }^{1} \mathrm{H}$ NMR $\left(400 \mathrm{MHz}, \mathrm{CDCl}_{3}\right) \delta=4.89(\mathrm{dd}, J=7.0,5.5 \mathrm{~Hz}, 1 \mathrm{H}) 4.42-4.71(\mathrm{~m}, 4 \mathrm{H}) 2.14$ (dd, $J=6.6,5.5 \mathrm{~Hz}, 1 \mathrm{H}) 2.04(\mathrm{~s}, 3 \mathrm{H}) 1.87(\mathrm{dd}, J=7.0,5.5 \mathrm{~Hz}, 1 \mathrm{H}) .{ }^{19} \mathrm{~F}$ NMR $(376 \mathrm{MHz}$, $\left.\mathrm{CDCl}_{3}\right) \delta=-73.8(\mathrm{t}, J=7.9 \mathrm{~Hz}, 3 \mathrm{~F}),-73.9(\mathrm{t}, J=7.9 \mathrm{~Hz}, 3 \mathrm{~F}) .{ }^{13} \mathrm{C} \mathrm{NMR}\left(150 \mathrm{MHz}, \mathrm{CDCl}_{3}\right)$ $\delta=169.9,165.9,163.3,122.4\left(\mathrm{~d}, J_{\mathrm{C}-\mathrm{F}}=277 \mathrm{~Hz}, 1 \mathrm{C}\right), 122.3\left(\mathrm{~d}, \mathrm{~J}_{\mathrm{C}-\mathrm{F}}=277 \mathrm{~Hz}, 1 \mathrm{C}\right), 61.4$ (q, $\left.J_{C-F}=37 \mathrm{~Hz}, 1 \mathrm{C}\right), 61.2\left(\mathrm{~d}, J_{\mathrm{C}-\mathrm{F}}=37 \mathrm{~Hz}, 1 \mathrm{C}\right), 57.5,33.4,20.5$, 20.2. FT-IR (thin film, $\mathrm{cm}^{-1}$ ): 3114, 3030, 2982, 1765, 1415, 1366, 1279, 1227, 1170, 1117, 976. HRMS calc'd for $\mathrm{C}_{11} \mathrm{H}_{10} \mathrm{~F}_{6} \mathrm{O}_{6}\left[\mathrm{M}^{+}\right]$: 352.0382; found: 352.0460 .

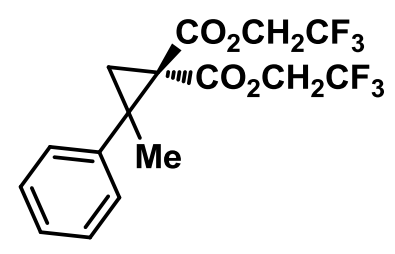

\section{Bis(2,2,2-trifluoroethyl) 2-methyl-2-phenylcyclopropane-1,1- dicarboxylate (2j).}

Following experimental procedure B2, cyclopropane $\mathbf{2 j}$ was prepared by dissolving commercially available $\alpha$-methylstyrene (200 mg, $1.70 \mathrm{mmol})$ and $\mathrm{Rh}_{2}(\mathrm{esp})_{2}(1.50 \mathrm{mg}, 0.002 \mathrm{mmol})$ in $5 \mathrm{~mL}$ of $\mathrm{CH}_{2} \mathrm{Cl}_{2}$ followed by the addition of bis(2,2,2-trifluoroethyl)2-diazomalonate ${ }^{2}(647 \mathrm{mg}, 2.20 \mathrm{mmol})$ dissolved in $1 \mathrm{~mL}$ of $\mathrm{CH}_{2} \mathrm{Cl}_{2}$. Cyclopropane $2 \mathrm{j}$ (648 $\mathrm{mg}$, quantitative yield) was obtained as a clear oil. $\mathrm{Rf}=0.60$ (30\% EtOAc in hexanes).

${ }^{1} \mathrm{H}$ NMR $\left(400 \mathrm{MHz}, \mathrm{CDCl}_{3}\right) \delta=7.32-7.23(\mathrm{~m}, 5 \mathrm{H}), 4.73-4.52(\mathrm{~m}, 2 \mathrm{H}), 4.16(\mathrm{dq}, J=$ 12.7, 8.3 Hz, 1H), 3.99 (dq, $J=12.7,8.3 \mathrm{~Hz}, 1 \mathrm{H}), 2.35(\mathrm{~d}, J=5.9 \mathrm{~Hz}, 1 \mathrm{H}), 1.86(\mathrm{~d}, J=$ $5.5 \mathrm{~Hz}, 1 \mathrm{H}), 1.58(\mathrm{~s}, 3 \mathrm{H}) .{ }^{19} \mathrm{~F}$ NMR $\left(376 \mathrm{MHz} \mathrm{CDCl}_{3}\right) \delta=-73.6(\mathrm{t}, J=7.9 \mathrm{~Hz}, 3 \mathrm{~F}),-73.8$ 
(t, $J=7.9 \mathrm{~Hz}, 3 \mathrm{~F}) .{ }^{13} \mathrm{C}$ NMR $\left(100 \mathrm{MHz}, \mathrm{CDCl}_{3}\right) \delta=166.2,165.5,139.8,128.5,128.1$, 127.6, $122.5\left(\mathrm{~d}, J_{\mathrm{C}-\mathrm{F}}=277 \mathrm{~Hz}, 1 \mathrm{C}\right), 122.0\left(\mathrm{~d}, J_{\mathrm{C}-\mathrm{F}}=277 \mathrm{~Hz}, 1 \mathrm{C}\right), 61.2\left(\mathrm{q}, J_{\mathrm{C}-\mathrm{F}}=37 \mathrm{~Hz}\right.$, 1C), 60.9 (q, $J_{C-F}=37 \mathrm{~Hz}, 1 C$ ), 40.1, 39.6, 25.7, 24.1. FT-IR (thin film, $\mathrm{cm}^{-1}$ ): 3063 , 2975, 2935, 1751, 1498, 1448, 1412, 1286, 1168, 1103, 977. HRMS calc'd for $\mathrm{C}_{16} \mathrm{H}_{14} \mathrm{~F}_{6} \mathrm{O}_{4}\left[\mathrm{M}^{+}\right]$: 384.0796; found: 384.0787 .

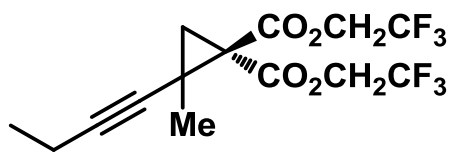

\section{Bis(2,2,2-trifluoroethyl) 2-(but-1-ynyl)-2-methylcyclopropane- 1,1-dicarboxylate (2k).}

Following experimental procedure B2, cyclopropane $\mathbf{2 k}$ was prepared by dissolving commercially available 2-methylhex-1-en-3-yne (500 mg, 5.30 $\mathrm{mmol}$ ) and $\mathrm{Rh}_{2}$ (esp) 2 (4.02 $\mathrm{mg}, 0.005 \mathrm{mmol}$ ) in $7 \mathrm{~mL}$ of $\mathrm{CH}_{2} \mathrm{Cl}_{2}$ followed by the addition of bis(2,2,2-trifluoroethyl)2-diazomalonate ${ }^{2}(2.03 \mathrm{~g}, 6.90 \mathrm{mmol})$ dissolved in $3 \mathrm{~mL}$ of $\mathrm{CH}_{2} \mathrm{Cl}_{2}$. Cyclopropane $\mathbf{2 k}$ (1.90 g, quantitative yield) was obtained as a colourless oil. $\mathrm{Rf}$ $=0.40(10 \%$ EtOAc in hexanes $)$.

${ }^{1} \mathrm{H}$ NMR $\left(400 \mathrm{MHz}, \mathrm{CDCl}_{3}\right) \delta=4.54(\mathrm{~m}, 4 \mathrm{H}), 2.13(\mathrm{q}, J=7.42,2 \mathrm{H}), 2.01(\mathrm{~d}, J=5.1 \mathrm{~Hz}$, $1 \mathrm{H}), 1.69(\mathrm{~d}, J=5.4 \mathrm{~Hz}, 1 \mathrm{H}), 1.49(\mathrm{~s}, 3 \mathrm{H}), 1.06(\mathrm{t}, J=7.4 \mathrm{~Hz}, 3 \mathrm{H}) .{ }^{19} \mathrm{~F}$ NMR $(376 \mathrm{MHz}$, $\left.\mathrm{CDCl}_{3}\right) \delta=-73.8(\mathrm{t}, J=8.6 \mathrm{~Hz}, 3 \mathrm{~F}),-73.8(\mathrm{t}, J=8.6 \mathrm{~Hz}, 3 \mathrm{~F}) .{ }^{13} \mathrm{C} \mathbf{N M R}\left(100 \mathrm{MHz}, \mathrm{CDCl}_{3}\right)$ $\delta=165.8,165.5,121.6\left(q, J_{C-F}=277 \mathrm{~Hz}, 1 \mathrm{C}\right), 121.5(q, J=277 \mathrm{~Hz}, 1 \mathrm{C}), 83.8,78.4$, $61.5\left(q, J_{C-F}=37 \mathrm{~Hz}, 1 \mathrm{C}\right), 61.3\left(q, J_{C-F}=37 \mathrm{~Hz}, 1 \mathrm{C}\right), 40.0,28.6,26.6,20.8,14.0,12.5$. FT-IR (thin film, $\mathrm{cm}^{-1}$ ): 2981, 2944, 2884, 2247, 2131, 1748, 1410, 1280, 1160, 1105. HRMS calc'd for $\mathrm{C}_{14} \mathrm{H}_{14} \mathrm{~F}_{6} \mathrm{O}_{4}\left[\mathrm{M}^{+}\right]$: 360.0796; found: 360.0789 .

\section{Experimental procedure C: Synthesis of azabicyclo[3.1.0]hexane-4,4- dicarboxylates (8b-k).}

Caution! Although we have never had any incidents, organic azides are potentially explosive substances that can decompose upon the exposure to heat, light, and pressure. Any azide synthesized should be stored in the freezer and in the dark. In addition, molecules containing the azido moiety can decompose violently which may result in injury if proper safety precautions are not taken. Reactions were performed with a blast shield.

NOTE: In addition to Dy(OTf)3, which was deemed the best, Lewis acids screened included $\left.\mathrm{Yb}(\mathrm{OTf})_{3}, \mathrm{Sc}(\mathrm{OTf})_{3}, \mathrm{Mgl}_{2}, \mathrm{CuOTf}\right)_{2}, \mathrm{TMSOTf}, \mathrm{BF}_{3} \mathrm{OEt} \mathrm{t}_{2}, \mathrm{Ni}\left(\mathrm{ClO}_{4}\right)_{2}, \mathrm{TiCl}_{4}$, $\mathrm{FeCl}_{3}, \mathrm{GaCl}_{3}$. 


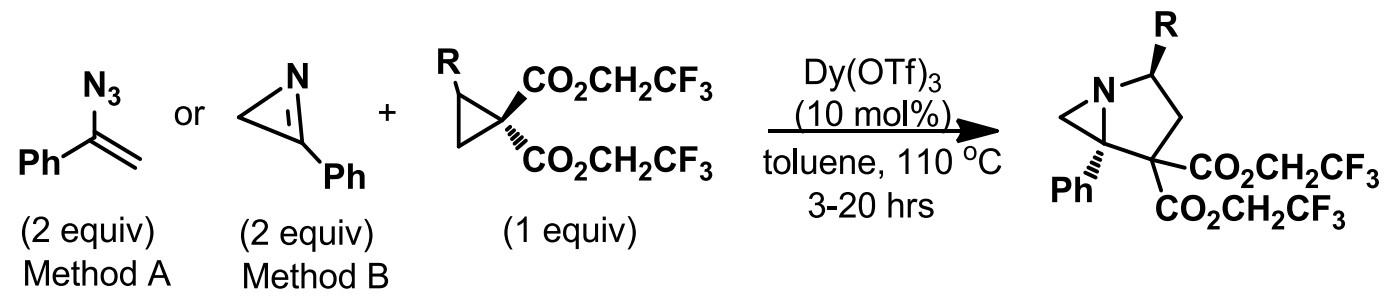

In a $10 \mathrm{~mL}$ round-bottomed flask, the appropriate bis(2,2,2-trifluoroethyl) cyclopropane1,1-dicarboxylate (1.0 equiv) and $\operatorname{Dy}(\mathrm{OTf})_{3}(10 \mathrm{~mol} \%)$ catalyst were dissolved in $6 \mathrm{~mL}$ of toluene. The reaction flask was fitted with a condenser and heated to $110{ }^{\circ} \mathrm{C}$. To this mixture, (1-azidovinyl)benzene ${ }^{4} \mathbf{5}$ (2.0 equiv) (Method A) or 3-phenyl-2H-azirine ${ }^{4} 6$ (2.0 equiv) (Method B), diluted in $1 \mathrm{~mL}$ of toluene, was added dropwise over a period of 1015 mins. The reaction was allowed to stir at $110^{\circ} \mathrm{C}$ for $3-20$ hours (monitored by TLC). The reaction flask was cooled to room temperature and the toluene was removed in vacuo. The crude reaction mixture was then pre-absorbed onto silica gel and purified by column chromatography (EtOAc in Hexanes).

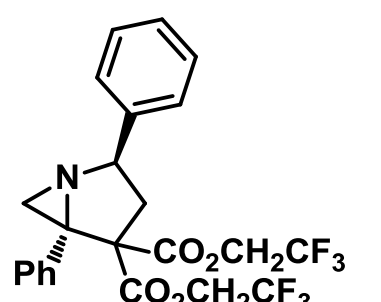

Bis(2,2,2-trifluoroethyl) 2,5-diphenyl-1-azabicyclo[3.1.0]hexane4,4-dicarboxylate (8b).

Following experimental procedure C Method A compound $\mathbf{8 b}$ was prepared by dissolving bis(2,2,2-trifluoroethyl) 2phenylcyclopropane-1,1-dicarboxylate ${ }^{3}(100 \mathrm{mg}, 0.270 \mathrm{mmol})$ and $\mathrm{Dy}(\mathrm{OTf})_{3}(16.8 \mathrm{mg}$, $0.027 \mathrm{mmol}$ ) in $6 \mathrm{~mL}$ of toluene. (1-azidovinyl)benzene 5 (78.3 $\mathrm{mg}, 0.540 \mathrm{mmol})$, in 1 $\mathrm{mL}$ of toluene, was added dropwise. The reaction was heated at $110{ }^{\circ} \mathrm{C}$ for 3.5 hours. Compound $8 \mathbf{b}$ (72 mg, $55 \%)$ was obtained as a clear oil. $\mathrm{Rf}=0.62(30 \%$ EtOAc in hexanes).

Following experimental procedure C Method B, compound $\mathbf{8 b}$ was prepared by dissolving bis(2,2,2-trifluoroethyl)2-phenylcyclopropane-1,1-dicarboxylate ${ }^{3}$ (150 mg, $0.405 \mathrm{mmol})$ and $\mathrm{Dy}(\mathrm{OTf})_{3}(25.0 \mathrm{mg}, 0.041 \mathrm{mmol})$ in $6 \mathrm{~mL}$ of toluene. 3-phenyl-2Hazirine $^{4} 6(95.0 \mathrm{mg}, 0.810 \mathrm{mmol})$, in $1 \mathrm{~mL}$ of toluene, was added dropwise. The reaction 
was heated at $110{ }^{\circ} \mathrm{C}$ for 10 hours. Compound $8 \mathrm{~b}(124 \mathrm{mg}, 63 \%)$ was obtained as a clear oil.

${ }^{1} \mathrm{H}$ NMR $\left(400 \mathrm{MHz}, \mathrm{CDCl}_{3}\right) \delta=7.61(\mathrm{~d}, J=1.0 \mathrm{~Hz}, 2 \mathrm{H}), 7.47(\mathrm{~d}, J=1.0 \mathrm{~Hz}, 2 \mathrm{H}), 7.40(\mathrm{t}$, $J=7.4 \mathrm{~Hz}, 2 \mathrm{H}$ ), $7.36-7.28(\mathrm{~m}, 4 \mathrm{H}), 5.35$ (dd, $J=10.9,7.0 \mathrm{~Hz}, 1 \mathrm{H}), 4.64(\mathrm{dq}, J=14.1$, $7.0 \mathrm{~Hz}, 1 \mathrm{H}), 4.52(\mathrm{dq}, J=12.5,8.2 \mathrm{~Hz}, 1 \mathrm{H}), 4.39(\mathrm{dq}, J=12.5,8.2 \mathrm{~Hz}, 1 \mathrm{H}), 3.77(\mathrm{dq}$, $J=12.5,8.2 \mathrm{~Hz}, 1 \mathrm{H}), 3.05(\mathrm{dd}, J=14.1,7.0 \mathrm{~Hz}, 1 \mathrm{H}), 2.23(\mathrm{dd}, J=13.9,11.1 \mathrm{~Hz}, 1 \mathrm{H}$ ), $2.16(\mathrm{~s}, 1 \mathrm{H}), 2.15(\mathrm{~s}, 1 \mathrm{H}) .{ }^{19} \mathrm{~F}$ NMR $\left(376 \mathrm{MHz}, \mathrm{CDCl}_{3}\right) \delta=-73.7(\mathrm{t}, J=8.6 \mathrm{~Hz}, 3 \mathrm{~F}),-$ 73.9 (t, $J=8.6 \mathrm{~Hz}, 3 \mathrm{~F}) .{ }^{13} \mathrm{C}$ NMR $\left(100 \mathrm{MHz}, \mathrm{CDCl}_{3}\right) \delta=168.1,167.9,139.7,136.8$, 128.7, 128.5, 128.1, 128.1, 127.3, 126.6, 126.3, 123.7 (d, $\left.J_{\mathrm{C}-\mathrm{F}}=277 \mathrm{~Hz}, 1 \mathrm{C}\right), 120.9$ (d, $\left.J_{C-F}=277 \mathrm{~Hz}, 1 \mathrm{C}\right), 66.2,65.1,61.3\left(q, J_{C-F}=37 \mathrm{~Hz}, 1 \mathrm{C}\right), 61.2\left(q, J_{C-F}=37 \mathrm{~Hz}, 1 \mathrm{C}\right)$, 54.9, 37.7, 32.8. FT-IR (thin film, $\mathrm{cm}^{-1}$ ): 3062, 3031, 1754, 1604, 1496, 1448, 1413, 1286, 1169, 1104, 980, 700. HRMS calc'd for $\mathrm{C}_{23} \mathrm{H}_{19} \mathrm{~F}_{6} \mathrm{NO}_{4}\left[\mathrm{M}^{+}\right]$: 487.1218; found: 487.1144.

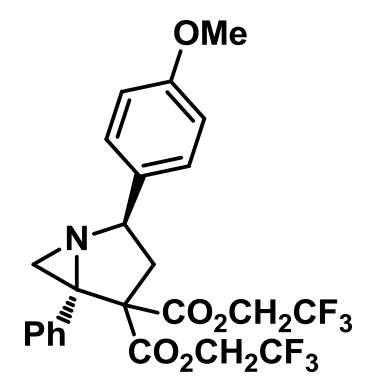

\section{Bis(2,2,2-trifluoroethyl) 2-(4-methoxyphenyl)-5-phenyl-1- azabicyclo[3.1.0]hexane- 4,4-dicarboxylate (8c).}

Following experimental procedure C Method A compound 8c was prepared by dissolving bis(2,2,2-trifluoroethyl) 2-(4methoxyphenyl)cyclopropane-1,1-dicarboxylate ${ }^{3}$ (150 mg, 0.375 $\mathrm{mmol})$ and $\mathrm{Dy}(\mathrm{OTf})_{3}(23.0 \mathrm{mg}, 0.037 \mathrm{mmol})$ in $6 \mathrm{~mL}$ of toluene. (1-azidovinyl)benzene ${ }^{4}$ 5 (108 $\mathrm{mg}, 0.750 \mathrm{mmol})$, in $1 \mathrm{~mL}$ of toluene, was added dropwise. The reaction was heated at $110{ }^{\circ} \mathrm{C}$ for 3 hours. Compound $8 \mathbf{c}(140 \mathrm{mg}, 72 \%)$ was obtained as a white solid. $\mathrm{MP}=98-100{ }^{\circ} \mathrm{C} . \mathrm{Rf}=0.56(30 \%$ EtOAc in hexanes $)$.

Following experimental procedure C Method B, compound $\mathbf{8 c}$ was prepared by dissolving bis(2,2,2-trifluoroethyl) 2-(4-methoxyphenyl)cyclopropane-1,1-dicarboxylate ${ }^{3}$ $(150 \mathrm{mg}, 0.375 \mathrm{mmol})$ and $\mathrm{Dy}(\mathrm{OTf})_{3}(23.0 \mathrm{mg}, 0.037 \mathrm{mmol})$ in $6 \mathrm{~mL}$ of toluene. 3phenyl-2H-azirine ${ }^{4} 6(87.8 \mathrm{mg}, 0.750 \mathrm{mmol})$, in $1 \mathrm{~mL}$ of toluene, was added dropwise. The reaction was heated at $110{ }^{\circ} \mathrm{C}$ for 14 hours. Compound $8 \mathrm{c}(179 \mathrm{mg}, 92 \%)$ was obtained as a white solid. 
${ }^{1} \mathrm{H}$ NMR $\left(400 \mathrm{MHz}, \mathrm{CDCl}_{3}\right) \delta=7.60(\mathrm{dd}, J=6.6,1.9 \mathrm{~Hz}, 2 \mathrm{H}), 7.36(\mathrm{~d}, J=8.2 \mathrm{~Hz}, 2 \mathrm{H})$, 7.24 - $7.34(\mathrm{~m}, 3 \mathrm{H}), 6.93(\mathrm{~d}, J=8.9 \mathrm{~Hz}, 2 \mathrm{H}), 5.27(\mathrm{dd}, J=11.1,7.2 \mathrm{~Hz}, 1 \mathrm{H}), 4.63(\mathrm{dq}, J$ $=12.8,8.2 \mathrm{~Hz}, 1 \mathrm{H}), 4.52(\mathrm{dq}, J=12.8,8.2 \mathrm{~Hz}, 1 \mathrm{H}), 4.37(\mathrm{dq}, J=12.5,8.2 \mathrm{~Hz}, 1 \mathrm{H}), 3.82$ (s, 3H), $3.76(\mathrm{dq}, J=12.8,8.2 \mathrm{~Hz}, 1 \mathrm{H}), 2.98(\mathrm{dd}, J=14.1,7.0 \mathrm{~Hz}, 1 \mathrm{H}), 2.20(\mathrm{dd}, J=$ 13.9, $11.1 \mathrm{~Hz}, 1 \mathrm{H}), 2.14(\mathrm{~s}, 1 \mathrm{H}), 2.09(\mathrm{~s}, 1 \mathrm{H}) .{ }^{19} \mathrm{~F} \mathrm{NMR}\left(376 \mathrm{MHz}, \mathrm{CDCl}_{3}\right) \delta=-73.74(\mathrm{t}$, $J=8.6 \mathrm{~Hz}, 3 \mathrm{~F}),-73.94(\mathrm{t}, J=8.6 \mathrm{~Hz}, 3 \mathrm{~F}) .{ }^{13} \mathrm{C}$ NMR $\left(100 \mathrm{MHz}, \mathrm{CDCl}_{3}\right) \delta=168.2,167.9$, 158.8, 136.8, 131.5, 128.7, 128.0, 127.8, 123.7 (d, JC-F $=277 \mathrm{~Hz}, 1 \mathrm{C}), 120.9$ (d, JC-F $=$ $277 \mathrm{~Hz}, 1 \mathrm{C}), 113.8,66.2,64.6,61.3\left(\mathrm{q}, J_{\mathrm{C}-\mathrm{F}}=37 \mathrm{~Hz}, 1 \mathrm{C}\right.$ ), 61.2 (q, JC-F $=37 \mathrm{~Hz}, 1 \mathrm{C}$ ), 55.3, 54.8, 37.7, 32.6, 30.8. FT-IR (thin film, $\mathrm{cm}^{-1}$ ): 2969, 2838, 1753, 1612, 1514, 1413, 1287, 1249, 1170, 1103, 981, 701. HRMS calc'd for $\mathrm{C}_{24} \mathrm{H}_{21} \mathrm{~F}_{6} \mathrm{NO}_{5}\left[\mathrm{M}^{+}\right]$: 517.1324; found: 517.1230 .

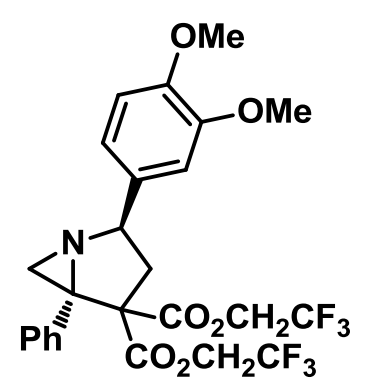

\section{Bis(2,2,2-trifluoroethyl) 2-(3,4-dimethoxyphenyl)-5-phenyl-1- azabicyclo[3.1.0] hexane-4,4-dicarboxylate (8d).}

Following experimental procedure C Method A compound 8d was prepared by dissolving cyclopropane 2d (155 mg, 0.360 $\mathrm{mmol})$ and $\mathrm{Dy}(\mathrm{OTf})_{3}(22 \mathrm{mg}, 0.036 \mathrm{mmol})$ in $6 \mathrm{~mL}$ of toluene. (1azidovinyl)benzene ${ }^{4} 5(104 \mathrm{mg}, 0.720 \mathrm{mmol})$, in $1 \mathrm{~mL}$ of toluene, was added dropwise. The reaction was heated at $110{ }^{\circ} \mathrm{C}$ for 8 hours. Compound $8 \mathrm{~d}(78 \mathrm{mg}, 40 \%)$ was obtained as a yellow oil. $\mathrm{Rf}=0.20$ (30\% EtOAc in hexanes).

Following experimental procedure C Method B, compound $\mathbf{8 d}$ was prepared by dissolving cyclopropane $2 \mathbf{d}(150 \mathrm{mg}, 0.348 \mathrm{mmol})$ and $\mathrm{Dy}(\mathrm{OTf})_{3}(21 \mathrm{mg}, 0.035 \mathrm{mmol})$ in $6 \mathrm{~mL}$ of toluene. 3-phenyl-2H-azirine $6(81.7 \mathrm{mg}, 0.697 \mathrm{mmol})$, in $1 \mathrm{~mL}$ of toluene, was added dropwise. The reaction was heated at $110{ }^{\circ} \mathrm{C}$ for 16 hours. Compound $8 \mathbf{d}$ (148 mg, $78 \%$ ) was obtained as a yellow oil.

${ }^{1} \mathrm{H}$ NMR $\left(400 \mathrm{MHz}, \mathrm{CDCl}_{3}\right) \delta=7.56(\mathrm{dd}, J=8.2,1.9 \mathrm{~Hz}, 2 \mathrm{H}), 7.31-7.21(\mathrm{~m}, 3 \mathrm{H}), 6.99$ (d, $J=2.0 \mathrm{~Hz}, 1 \mathrm{H}$ ), 6.89 (dd, $J=8.2,1.9 \mathrm{~Hz}, 1 \mathrm{H}$ ), $6.84(\mathrm{~s}, 1 \mathrm{H}), 5.24$ (dd, $J=10.9,7.4$ $\mathrm{Hz}, 1 \mathrm{H}), 4.60$ (dq, $J=16.4,8.2 \mathrm{~Hz}, 1 \mathrm{H}), 4.49(\mathrm{dq}, J=16.4,8.2 \mathrm{~Hz}, 1 \mathrm{H}), 4.35(\mathrm{dq}, J=$ 12.5, $8.2 \mathrm{~Hz}, 1 \mathrm{H}$ ), 3.88 (s, 3H), 3.86 (s, 3H), 3.72 (dq, $J=12.5,8.2 \mathrm{~Hz}, 1 \mathrm{H}$ ), 2.96 (dd, $J$ $=14.1,7.0 \mathrm{~Hz}, 1 \mathrm{H}), 2.18(\mathrm{dd}, J=13.9,11.1 \mathrm{~Hz}, 1 \mathrm{H}), 2.11(\mathrm{~s}, 1 \mathrm{H}), 2.07(\mathrm{~s}, 1 \mathrm{H}) .{ }^{19} \mathrm{~F}$ NMR 
$\left(376 \mathrm{MHz}, \mathrm{CDCl}_{3}\right) \delta=-73.7$ (t, $\left.J=8.6 \mathrm{~Hz}, 3 \mathrm{~F}\right),-73.9$ (t, $\left.J=8.6 \mathrm{~Hz}, 3 \mathrm{~F}\right) .{ }^{13} \mathrm{C}$ NMR $\left(100 \mathrm{MHz}, \mathrm{CDCl}_{3}\right) \delta=168.2,167.8,148.9,148.3,136.8,132.2,128.7,128.1,122.5$ (q, $\left.J_{C-F}=277 \mathrm{~Hz}, 1 \mathrm{C}\right), 122.1\left(\mathrm{q}, J_{\mathrm{C}-\mathrm{F}}=277 \mathrm{~Hz}, 1 \mathrm{C}\right), 118.3,111.0,110.5,66.2,64.8,61.3$ (q, $\left.J_{\text {C-F }}=37 \mathrm{~Hz}, 1 \mathrm{C}\right), 63.2\left(\mathrm{q}, \mathrm{J}_{\mathrm{C}-\mathrm{F}}=37 \mathrm{~Hz}, 1 \mathrm{C}\right), 56.0,55.9,54.8,37.6,32.6$. FT-IR (thin film, $\left.\mathrm{cm}^{-1}\right)$ : 3062, 3004, 2965, 2838, 1753, 1518, 1414, 1285, 1242, 1168, 1103, 1028, 975. HRMS calc'd for $\mathrm{C}_{25} \mathrm{H}_{23} \mathrm{~F}_{6} \mathrm{NO}_{6}\left[\mathrm{M}^{+}\right]: 547.1430$ found; 547.1451 .

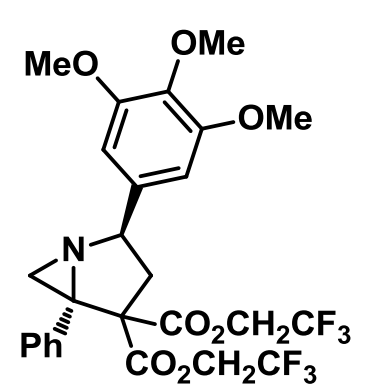

Bis(2,2,2-trifluoroethyl) 5-phenyl-2-(3,4,5-trimethoxyphenyl)-1azabicyclo[3.1.0] hexane-4,4-dicarboxylate (8e).

Following experimental procedure C Method A, compound $\mathbf{8 e}$ was prepared by dissolving cyclopropane $2 \mathrm{e}(150 \mathrm{mg}, 0.326 \mathrm{mmol})$ and $\mathrm{Dy}(\mathrm{OTf})_{3} \quad(20 \mathrm{mg}, \quad 0.033 \mathrm{mmol})$ in $6 \mathrm{~mL}$ of toluene. (1azidovinyl)benzene 5 (94.5 mg, $0.652 \mathrm{mmol}$ ), in $1 \mathrm{~mL}$ of toluene, was added dropwise. The reaction was heated at $110{ }^{\circ} \mathrm{C}$ for 8 hours. Compound $8 \mathrm{e}(96 \mathrm{mg}, 51 \%)$ was obtained as a white solid. $\mathrm{MP}=111-113^{\circ} \mathrm{C} . \mathrm{Rf}=0.24$ (30\% EtOAc in hexanes).

Following experimental procedure $\mathrm{C}$ Method $\mathrm{B}$, compound $\mathbf{8 e}$ was prepared by dissolving cyclopropane $2 \mathrm{e}(150 \mathrm{mg}, 0.326 \mathrm{mmol})$ and Dy $(\mathrm{OTf})_{3}(20 \mathrm{mg}, 0.033 \mathrm{mmol})$ in $6 \mathrm{~mL}$ of toluene. 3-phenyl-2H-azirine ${ }^{4} 6(76.3 \mathrm{mg}, 0.652 \mathrm{mmol})$, in $1 \mathrm{~mL}$ of toluene, was added dropwise. The reaction was heated at $110^{\circ} \mathrm{C}$ for 18 hours. Compound $8 \mathbf{e}$ (158 $\mathrm{mg}, 84 \%$ ) was obtained as a white solid.

${ }^{1} \mathrm{H}$ NMR $\left(400 \mathrm{MHz}, \mathrm{CDCl}_{3}\right) \delta=7.56-7.60(\mathrm{~m}, 2 \mathrm{H}), 7.34-7.24(\mathrm{~m}, 3 \mathrm{H}), 6.64(\mathrm{~d}, J=0.8$ $\mathrm{Hz}, 2 \mathrm{H}$ ), 5.26 (dd, $J=10.9,7.4 \mathrm{~Hz}, 1 \mathrm{H}), 4.62(\mathrm{qd}, J=16.4,8.2 \mathrm{~Hz}, 1 \mathrm{H}), 4.52(\mathrm{dq}, J=$ 16.4, $8.2 \mathrm{~Hz}, 1 \mathrm{H}$ ), 4.38 (dq, $J=12.6,8.3 \mathrm{~Hz}, 1 \mathrm{H}$ ), 3.87 (s, 6H), $3.84(\mathrm{~s}, 3 \mathrm{H}), 3.75$ (dq, J $=12.5,8.2 \mathrm{~Hz}, 1 \mathrm{H}), 3.00(\mathrm{dd}, J=13.9,7.2 \mathrm{~Hz}, 1 \mathrm{H}), 2.19(\mathrm{dd}, J=14.0,10.9 \mathrm{~Hz}, 1 \mathrm{H}$ ), $2.15(\mathrm{~s}, 3 \mathrm{H}), 2.14(\mathrm{~d}, J=3.1 \mathrm{~Hz}, 2 \mathrm{H}) .{ }^{19} \mathrm{~F}$ NMR $\left(376 \mathrm{MHz}, \mathrm{CDCl}_{3}\right) \delta=-73.7(\mathrm{t}, J=8.6$ $\mathrm{Hz}, 3 \mathrm{~F}),-73.9$ (t, $J=8.6 \mathrm{~Hz}, 3 \mathrm{~F}) .{ }^{13} \mathrm{C}$ NMR $\left(100 \mathrm{MHz}, \mathrm{CDCl}_{3}\right) \delta=168.0,167.7,153.2$, 137.2, 136.6, 135.4, 128.7, 128.1, 122.4 (q, JC-F $=277 \mathrm{~Hz}, 1 \mathrm{C}$ ), 122.0 (q, JC-F $=277 \mathrm{~Hz}$, 1C), 103.8, 66.7, 65.1, $61.3\left(q, J_{C-F}=37 \mathrm{~Hz}, 1 \mathrm{C}\right), 61.2\left(q, J_{c-F}=37 \mathrm{~Hz}, 1 \mathrm{C}\right), 60.7,56.2$, 
54.8, 37.7, 32.8. FT-IR (thin film, $\mathrm{cm}^{-1}$ ): 3061, 2969, 2942, 1753, 1589, 1509, 1416, 1286, 1241, 1168, 1128, 977, 701. HRMS calc'd for $\mathrm{C}_{26} \mathrm{H}_{25} \mathrm{~F}_{6} \mathrm{NO}_{7}\left[\mathrm{M}^{+}\right]$: 577.1535; found: 577.1560 .

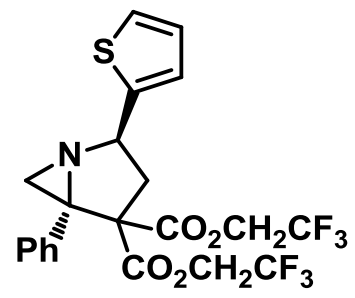

\section{Bis(2,2,2-trifluoroethyl) 5-phenyl-2-(thiophen-2-yl)-1- azabicyclo[3.1.0]hexane-4,4-dicarboxylate (8f).}

Following experimental procedure C Method A, compound $8 \mathrm{f}$ was prepared by dissolving bis(2,2,2-trifluoroethyl)2-(thiophen-2yl)cyclopropane-1,1-dicarboxylate $2 f(150 \mathrm{mg}, 0.399 \mathrm{mmol})$ and $\mathrm{Dy}(\mathrm{OTf})_{3}(24 \mathrm{mg}, 0.039 \mathrm{mmol})$ in $6 \mathrm{~mL}$ of toluene. (1-azidovinyl)benzene ${ }^{4} 5(115 \mathrm{mg}$, $0.798 \mathrm{mmol}$ ), in $1 \mathrm{~mL}$ of toluene, was added dropwise. The reaction was heated at 110 ${ }^{\circ} \mathrm{C}$ for 12 hours Compound $8 \mathrm{f}$ ( $155 \mathrm{mg}, 80 \%$ ) was obtained as a yellow oil. $\mathrm{Rf}=0.48$ (30\% EtOAc in hexanes).

Following experimental procedure C Method B, compound $8 \mathbf{f}$ was prepared by dissolving bis(2,2,2-trifluoroethyl) 2-(thiophen-2-yl)cyclopropane-1,1-dicarboxylate $2 \mathbf{2 f}$ $(150 \mathrm{mg}, 0.399 \mathrm{mmol})$ and $\mathrm{Dy}(\mathrm{OTf})_{3}(24 \mathrm{mg}, 0.039 \mathrm{mmol})$ in $6 \mathrm{~mL}$ of toluene. 3-phenyl$2 \mathrm{H}$-azirine ${ }^{4} 6(93.4 \mathrm{mg}, 0.798 \mathrm{mmol})$, in $1 \mathrm{~mL}$ of toluene, was added dropwise. The reaction was heated at $110{ }^{\circ} \mathrm{C}$ for 18 hours. Compound $8 f(157 \mathrm{mg}, 80 \%)$ was obtained as a yellow oil.

${ }^{1} \mathrm{H}$ NMR $\left(400 \mathrm{MHz}, \mathrm{CDCl}_{3}\right) \delta=7.59-7.55(\mathrm{~m}, 2 \mathrm{H}), 7.32-7.23(\mathrm{~m}, 4 \mathrm{H}), 7.05-6.98(\mathrm{~m}$, 2H), 5.43 (dd, $J=10.7,6.8 \mathrm{~Hz}, 1 \mathrm{H}$ ), $4.64(\mathrm{dq}, J=12.5,8.2 \mathrm{~Hz}, 1 \mathrm{H}), 4.52(\mathrm{dq}, J=16.4$, $8.2 \mathrm{~Hz}, 1 \mathrm{H}$ ), 4.35 (dq, $J=12.5,8.2 \mathrm{~Hz}, 1 \mathrm{H}$ ), 3.75 (dq, $J=16.4,8.2 \mathrm{~Hz}, 1 \mathrm{H}), 2.98$ (dd, $J$ $=14.1,7.0 \mathrm{~Hz}, 1 \mathrm{H}), 2.28(\mathrm{dd}, J=14.1,7.0 \mathrm{~Hz}, 1 \mathrm{H}), 2.26(\mathrm{~d}, J=1.2 \mathrm{~Hz}, 1 \mathrm{H}), 2.06(\mathrm{~d}, J$ $=1.2 \mathrm{~Hz}, 1 \mathrm{H}) .{ }^{19} \mathrm{~F}$ NMR $\left(376 \mathrm{MHz}, \mathrm{CDCl}_{3}\right) \delta=-73.74(\mathrm{t}, J=8.6 \mathrm{~Hz}, 3 \mathrm{~F}),-73.94$ (t, $J=$ $8.6 \mathrm{~Hz}, 3 \mathrm{~F}) .{ }^{13} \mathrm{C}$ NMR $\left(100 \mathrm{MHz}, \mathrm{CDCl}_{3}\right) \delta=168.0,167.6,142.1,136.5,128.6,128.2$, 128.1, 126.7, 124.9, 124.8, 122.5 (q, JC-F $=277 \mathrm{~Hz}, 1 \mathrm{C}$ ), 122.1 (q, JC-F $=277 \mathrm{~Hz}, 1 \mathrm{C}$ ), 66.2, 62.0, $61.3\left(\mathrm{q}, J_{\mathrm{C}-\mathrm{F}}=37 \mathrm{~Hz}, 1 \mathrm{C}\right), 63.2\left(\mathrm{q}, J_{\mathrm{C}-\mathrm{F}}=37 \mathrm{~Hz}, 1 \mathrm{C}\right), 55.4,38.6$, 32.8. FT-IR (thin film, $\mathrm{cm}^{-1}$ ): 3064, 2973, 1754, 1448, 1413, 1286, 1247, 1170, 1103, 978, 701. HRMS calc'd for $\mathrm{C}_{21} \mathrm{H}_{17} \mathrm{~F}_{6} \mathrm{NO}_{4} \mathrm{~S}\left[\mathrm{M}^{+}\right]$: 493.0782; found: 493.0721 . 


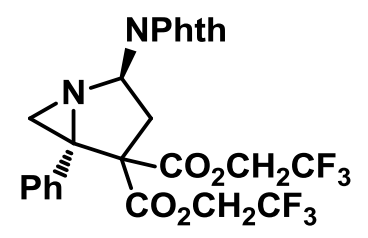

Bis(2,2,2-trifluoroethyl) 2-(1,3-dioxoisoindolin-2-yl)-5-phenyl-1azabicyclo[3.1.0] hexane-4,4-dicarboxylate (8g).

was prepared by dissolving cyclopropane $\mathbf{2 g}(150 \mathrm{mg}, 0.342 \mathrm{mmol})$ and $\mathrm{Dy}(\mathrm{OTf})_{3}(21$ $\mathrm{mg}, 0.034 \mathrm{mmol}$ ) in $6 \mathrm{~mL}$ of toluene. (1-azidovinyl)benzene 5 (99 mg, $0.684 \mathrm{mmol}$ ), in 1 $\mathrm{mL}$ of toluene, was then added dropwise. The reaction was heated at $110{ }^{\circ} \mathrm{C}$ for 16 hours. Compound $8 \mathbf{g}(70 \mathrm{mg}, 37 \%)$ was obtained as a yellow solid. MP $=151-153^{\circ} \mathrm{C}$. Rf $=0.41$ (30\% EtOAc in hexanes).

Following experimental procedure C Method $\mathbf{B}$, compound $\mathbf{8 g}$ was prepared by dissolving cyclopropane $\mathbf{2 g}(150 \mathrm{mg}, 0.342 \mathrm{mmol})$ and $\mathrm{Dy}(\mathrm{OTf})_{3}(21 \mathrm{mg}, 0.034 \mathrm{mmol})$ in $6 \mathrm{~mL}$ of toluene. 3-phenyl-2H-azirine ${ }^{4} 6(80 \mathrm{mg}, 0.684 \mathrm{mmol})$, in $1 \mathrm{~mL}$ of toluene, was added dropwise. The reaction was heated at $110^{\circ} \mathrm{C}$ for 20 hours. Compound $8 \mathrm{~g}$ (140 $\mathrm{mg}, 74 \%$ ) was obtained as a yellow solid.

${ }^{1} \mathrm{H}$ NMR $\left(400 \mathrm{MHz}, \mathrm{CDCl}_{3}\right) \delta=7.92(\mathrm{~d}, J=2.7 \mathrm{~Hz}, 1 \mathrm{H}), 7.90(\mathrm{~d}, J=3.1 \mathrm{~Hz}, 1 \mathrm{H}), 7.79$ (d, $J=3.1 \mathrm{~Hz}, 1 \mathrm{H}), 7.77(\mathrm{~d}, J=3.1 \mathrm{~Hz}, 1 \mathrm{H}), 7.61-7.57(\mathrm{~m}, 2 \mathrm{H}), 7.34-7.25(\mathrm{~m}, 5 \mathrm{H})$, 6.36 (dd, $J=10.9,7.0 \mathrm{~Hz}, 1 \mathrm{H}$ ), $4.71-4.55(\mathrm{~m}, 2 \mathrm{H}), 4.35$ (dq, $J=12.5,8.3 \mathrm{~Hz}, 1 \mathrm{H}$ ), 3.73 (dq, $J=12.5,8.2 \mathrm{~Hz}, 1 \mathrm{H}$ ), $3.48(\mathrm{dd}, J=14.1,10.9 \mathrm{~Hz}, 1 \mathrm{H}$ ), $3.20(\mathrm{~d}, J=1.2 \mathrm{~Hz}, 1 \mathrm{H}) 2.68$ $(\mathrm{dd}, J=14.1,7.0 \mathrm{~Hz}, 1 \mathrm{H}) 2.15(\mathrm{~d}, J=1.6 \mathrm{~Hz}, 1 \mathrm{H}) .{ }^{19} \mathrm{~F}$ NMR $\left(376 \mathrm{MHz}, \mathrm{CDCl}_{3}\right) \delta=-$ $73.74(\mathrm{t}, J=8.6 \mathrm{~Hz}, 3 \mathrm{~F}),-73.94(\mathrm{t}, J=8.6 \mathrm{~Hz}, 3 \mathrm{~F}) .{ }^{13} \mathrm{C} \mathbf{N M R}\left(100 \mathrm{MHz}, \mathrm{CDCl}_{3}\right) \delta=$ 168.2, 167.8, 167.3, 135.8, 134.5, 131.5, 128.6, 128.3, 128.1, 123.8, 123.6, 68.8, 64.9, $61.4\left(q, J_{C-F}=37 \mathrm{~Hz}, 1 \mathrm{C}\right), 61.3\left(q, J_{C-F}=37 \mathrm{~Hz}, 1 \mathrm{C}\right), 52.9,35.1,30.7$. FT-IR (thin film, $\mathrm{cm}^{-1}$ ): 3030, 2979, 2880, 1777, 1719, 1604, 1448, 1373, 1284, 1169, 996, 717. HRMS calc'd for $\mathrm{C}_{25} \mathrm{H}_{18} \mathrm{~F}_{6} \mathrm{~N}_{2} \mathrm{O}_{6}\left[\mathrm{M}^{+}\right]$]: 556.1069; found: 556.1005 .

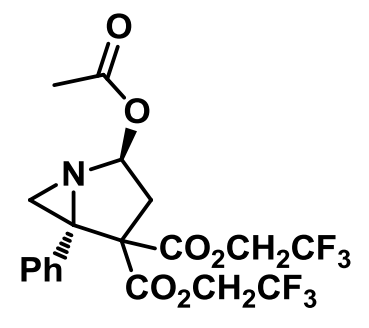

\section{Bis(2,2,2-trifluoroethyl) 2-acetoxy-5-phenyl-1-azabicyclo[3.1.0] hexane-4,4-dicarboxylate (8h).}

Following experimental procedure C Method B, compound $8 \mathrm{~h}$ was prepared by dissolving cyclopropane $2 \mathrm{~h}(150 \mathrm{mg}, 0.426 \mathrm{mmol})$ and 
$\mathrm{Dy}(\mathrm{OTf})_{3}(26 \mathrm{mg}, 0.043 \mathrm{mmol})$ in $6 \mathrm{~mL}$ of toluene. 3-phenyl-2H-azirine ${ }^{4} 5$ (100 mg, $0.852 \mathrm{mmol}$ ), in $1 \mathrm{~mL}$ of toluene, was added dropwise. The reaction was heated at 110 ${ }^{\circ} \mathrm{C}$ for 16 hours. Compound $8 \mathrm{~h}(67 \mathrm{mg}, 34 \%$ ) was obtained as a yellow oil. $\mathrm{Rf}=0.3$ (30\% EtOAc in hexanes).

${ }^{1} \mathbf{H}$ NMR $\left(400 \mathrm{MHz}, \mathrm{CDCl}_{3}\right) \delta=7.54-7.50(\mathrm{~m}, 2 \mathrm{H}), 7.30-7.23(\mathrm{~m}, 3 \mathrm{H}), 6.47(\mathrm{dd}, J=$ 7.9, $6.7 \mathrm{~Hz}, 1 \mathrm{H}$ ), $4.65(\mathrm{dq}, J=12.5,8.3 \mathrm{~Hz}, 1 \mathrm{H}$ ), 4.49 (dq, $J=12.5,8.3 \mathrm{~Hz}, 1 \mathrm{H}$ ), 4.29 (dq, $J=12.5,8.2 \mathrm{~Hz}, 1 \mathrm{H}$ ), 3.71 (dq, $J=12.5,8.2 \mathrm{~Hz}, 1 \mathrm{H}$ ), 2.97 (dd, $J=14.4,6.7 \mathrm{~Hz}$, $1 \mathrm{H}), 2.13(\mathrm{~d}, J=1.8 \mathrm{~Hz}, 1 \mathrm{H}), 2.12(\mathrm{~s}, 3 \mathrm{H}), 2.04(\mathrm{dd}, J=14.4,8.5 \mathrm{~Hz}, 1 \mathrm{H}) .{ }^{19} \mathrm{~F}$ NMR $\left(376 \mathrm{MHz}, \mathrm{CDCl}_{3}\right) \delta=-73.74(\mathrm{t}, J=8.6 \mathrm{~Hz}, 3 \mathrm{~F}),-73.94(\mathrm{t}, J=8.6 \mathrm{~Hz}, 3 \mathrm{~F}) .{ }^{13} \mathrm{C} \mathbf{N M R}$ $\left(100 \mathrm{MHz}, \mathrm{CDCl}_{3}\right) \delta=169.4,167.5,167.2,135.7,128.5,128.1,122.4$ (q, JC-F $=277 \mathrm{~Hz}$, 1C), 121.9 (q, JC-F $=277 \mathrm{~Hz}, 1 \mathrm{C}), 87.6,64.5,61.4\left(\mathrm{q}, J_{\mathrm{C}-\mathrm{F}}=37 \mathrm{~Hz}, 1 \mathrm{C}\right), 61.3\left(\mathrm{q}, J_{\mathrm{C}-\mathrm{F}}=\right.$ $37 \mathrm{~Hz}, 1 \mathrm{C}$ ), 54.2, 36.3, 33.0, 20.8. FT-IR (thin film, $\mathrm{cm}^{-1}$ ): 3064, 3031, 2975, 1755, 1496, 1448, 1413, 1285, 1111, 1031, 979. HRMS calc'd for $\mathrm{C}_{19} \mathrm{H}_{17} \mathrm{~F}_{6} \mathrm{NO}_{6}\left[\mathrm{M}^{+}\right]$: 469.0960; found: 469.0899 .

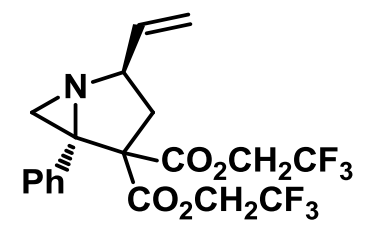

Bis(2,2,2-trifluoroethyl) 5-phenyl-2-vinyl-1-azabicyclo[3.1.0] hexane-4,4-dicarboxylate (8i).

was prepared by dissolving bis(2,2,2-trifluoroethyl) 2-vinylcyclopropane-1,1dicarboxylate $^{3}(123 \mathrm{mg}, 0.384 \mathrm{mmol})$ and $\mathrm{Dy}(\mathrm{OTf})_{3}(23 \mathrm{mg}, 0.040 \mathrm{mmol})$ in $6 \mathrm{~mL}$ of toluene. (1-azidovinyl)benzene 5 (112 $\mathrm{mg}, 0.768 \mathrm{mmol})$, in $1 \mathrm{~mL}$ of toluene, was added dropwise. The reaction was heated at $110^{\circ} \mathrm{C}$ for 12 hours. Compound $8 \mathbf{i}(45 \mathrm{mg}$, $27 \%$ ) was obtained as a clear oil. $\mathrm{Rf}=0.52$ (30\% EtOAc in hexanes).

Following experimental procedure C Method B, compound $8 \mathbf{i}$ was prepared by dissolving bis(2,2,2-trifluoroethyl) 2-vinylcyclopropane-1,1-dicarboxylate ${ }^{3}$ (128 mg, $0.400 \mathrm{mmol})$ and $\mathrm{Dy}(\mathrm{OTf})_{3}(24 \mathrm{mg}, 0.040 \mathrm{mmol})$ in $6 \mathrm{~mL}$ of toluene. 3-phenyl-2Hazirine ${ }^{4} 6(93.6 \mathrm{mg}, 0.833 \mathrm{mmol})$, in $1 \mathrm{~mL}$ of toluene, was added dropwise. The reaction was heated at $110{ }^{\circ} \mathrm{C}$ for 15 hours. Compound $8 \mathbf{i}$ (44 mg, 25\%) was obtained as a clear oil. 
${ }^{1} \mathrm{H}$ NMR $\left(400 \mathrm{MHz}, \mathrm{CDCl}_{3}\right) \delta=7.56-7.52(\mathrm{~m}, 2 \mathrm{H}), 7.32-7.25(\mathrm{~m}, 3 \mathrm{H}), 6.00-5.91(\mathrm{~m}$, $1 \mathrm{H}$ ), $5.36(\mathrm{dt}, J=17.2,1.4 \mathrm{~Hz}, 1 \mathrm{H}$ ), $5.26(\mathrm{dt}, J=10.6,1.4 \mathrm{~Hz}, 1 \mathrm{H}), 4.65(\mathrm{dq}, J=12.5$, $8.3 \mathrm{~Hz}, 2 \mathrm{H}$ ), 4.50 (dq, $J=12.5,8.2 \mathrm{~Hz}, 1 \mathrm{H}$ ), 4.33 (dq, $J=2.5,8.3 \mathrm{~Hz}, 1 \mathrm{H}$ ), 3.74 (dq, $J=$ 12.7, 8.3 Hz, 1H), 2.67 (dd, J=14.5, 7.0 Hz, 1H), 2.13 (s, 1H), 2.01 (s, 1H), 1.96 (dd, J $=14.1,10.9 \mathrm{~Hz}, 1 \mathrm{H}) .{ }^{19} \mathrm{~F} \mathrm{NMR}\left(376 \mathrm{MHz}, \mathrm{CDCl}_{3}\right) \delta=-73.77(\mathrm{t}, J=8.6 \mathrm{~Hz}, 3 \mathrm{~F}),-73.99$ (t, $J=8.6 \mathrm{~Hz}, 3 \mathrm{~F}) .{ }^{3} \mathrm{C}$ NMR $\left(100 \mathrm{MHz}, \mathrm{CDCl}_{3}\right) \delta=168.0,167.9,136.7,135.3,128.6$, 128.0, 122.5 (q, JC-F $=277 \mathrm{~Hz}, 1 \mathrm{C}$ ), 122.1 (q, JC-F $=277 \mathrm{~Hz}, 1 \mathrm{C}$ ), 117.3, 65.9, 64.6, 61.2 (q, JC-F $=36 \mathrm{~Hz}, 1 \mathrm{C}$ ), 61.1 (q, JC-F $=37 \mathrm{~Hz}, 1 \mathrm{C}$ ), 54.5, 36.9, 32.2. FT-IR (thin film, $\mathrm{cm}^{-1}$ ): $3063,2976,1754,1496,1448,1413,1286,1233,1170,1103,977,701$. HRMS calc'd for $\mathrm{C}_{19} \mathrm{H}_{17} \mathrm{~F}_{6} \mathrm{NO}_{4}\left[\mathrm{M}^{+}\right]$: 437.1062 ; found: 437.0990 .

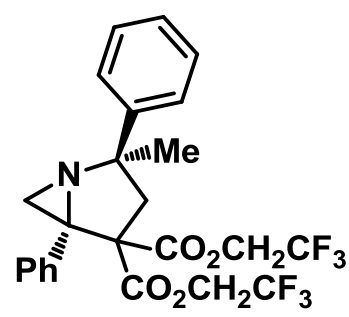

\section{Bis(2,2,2-trifluoroethyl) 2-methyl-2,5-diphenyl-1-azabicyclo[3.1.0] hexane-4,4-dicarboxylate (8j).}

Following experimental procedure C Method A, compound 8j was prepared by dissolving cyclopropane $2 \mathbf{j}(150 \mathrm{mg}, 0.390 \mathrm{mmol}$ ) and $\operatorname{Dy}(\mathrm{OTf})_{3} \quad(24 \mathrm{mg}, \quad 0.039 \mathrm{mmol})$ in $6 \mathrm{~mL}$ of toluene. (1azidovinyl)benzene ${ }^{4} 5$ (113 $\mathrm{mg}, 0.781 \mathrm{mmol}$ ), in $1 \mathrm{~mL}$ of toluene, was added dropwise. The reaction was heated at $110{ }^{\circ} \mathrm{C}$ for 12 hours. Compound $8 \mathbf{j}(160 \mathrm{mg}, 82 \%$ ) was obtained as a yellow semi-solid in a 1:1 mixture of diastereomers. $\mathrm{Rf}=0.80(30 \%$ EtOAc in hexanes).

Following experimental procedure C Method B, compound 8j was prepared by dissolving cyclopropane $2 \mathbf{j}(150 \mathrm{mg}, 0.390 \mathrm{mmol})$ and $\mathrm{Dy}(\mathrm{OTf})_{3}(24 \mathrm{mg}, 0.039 \mathrm{mmol})$ in $6 \mathrm{~mL}$ of toluene. 3-phenyl-2H-azirine ${ }^{4} 6(91 \mathrm{mg}, 0.781 \mathrm{mmol})$, in $1 \mathrm{~mL}$ of toluene, was added dropwise. The reaction was heated at $110{ }^{\circ} \mathrm{C}$ for 18 hours. Compound $8 \mathbf{j}$ (169 $\mathrm{mg}, 87 \%$ ) was obtained as a yellow semi-solid in a 1:1 mixture of diastereomers.

${ }^{1} \mathrm{H}$ NMR (400 MHz, $\mathrm{CDCl}_{3}$ ) (mixture of diastereomers) $\delta=7.83(\mathrm{~d}, J=8.6 \mathrm{~Hz}, 2 \mathrm{H}), 7.62$ (d, $J=8.6 \mathrm{~Hz}, 2 \mathrm{H}), 7.53(\mathrm{~d}, J=8.2 \mathrm{~Hz}, 2 \mathrm{H}), 7.46(\mathrm{t}, J=8.6 \mathrm{~Hz}, 2 \mathrm{H}), 7.43-7.36(\mathrm{~m}, 5$ H), 7.35- $7.23(\mathrm{~m}, 7 \mathrm{H}), 4.76-4.63(\mathrm{~m}, 1 \mathrm{H}), 4.62-4.41(\mathrm{~m}, 4 \mathrm{H}), 3.94(\mathrm{dq}, J=16.4,8.2$ $\mathrm{Hz}, 1 \mathrm{H}), 3.68(\mathrm{dq}, J=12.6,8.3 \mathrm{~Hz}, 1 \mathrm{H}), 3.46(\mathrm{dq}, J=12.5,8.4 \mathrm{~Hz}, 1 \mathrm{H}), 3.31(\mathrm{~d}, J=$ $14.8 \mathrm{~Hz}, 1 \mathrm{H}), 3.25(\mathrm{~d}, J=14.5 \mathrm{~Hz}, 1 \mathrm{H}), 2.64(\mathrm{~d}, J=14.5 \mathrm{~Hz}, 1 \mathrm{H}), 2.59(\mathrm{~d}, J=14.5 \mathrm{~Hz}, 1$ 
$\mathrm{H}), 2.41(\mathrm{~s}, 1 \mathrm{H}), 2.26(\mathrm{~s}, 1 \mathrm{H}), 2.13(\mathrm{~s}, 1 \mathrm{H}), 1.90(\mathrm{~s}, 3 \mathrm{H}), 1.87(\mathrm{~s}, 1 \mathrm{H}), 1.57(\mathrm{~s}, 3 \mathrm{H}) .{ }^{19} \mathrm{~F}$ NMR $\left(376 \mathrm{MHz}, \mathrm{CDCl}_{3}\right.$ ) (mixture of diastereomers) $\delta=.73 .64$ (t, J=8.6 Hz, 3F), -73.71 (t, J=8.6 Hz, 3F), $-73.99--73.89$ (m, 6F). ${ }^{13} \mathrm{C}$ NMR $\left(100 \mathrm{MHz}, \mathrm{CDCl}_{3}\right.$ ) (mixture of diastereomers) $\delta=168.6,168.3,168.1,166.9$, 147.8, 147.0, 138.1, 137.3, 128.9, 128.4, 128.2, 128.1, 128.0, 127.9, 127.4, 126.6, 125.8, 125.6, 122.5 (d, JC-F $=277 \mathrm{~Hz}, 2 \mathrm{C}$ ), 122.1 (q, JC-F $=277 \mathrm{~Hz}, 1 \mathrm{C}$ ), 122.0 (q, JC-F $=277 \mathrm{~Hz}, 1 \mathrm{C}$ ), 70.9, 69.5, 66.8, 65.3, 61.3 (q, JC-F $=37 \mathrm{~Hz}, 2 \mathrm{C}$ ), 61.2 (q, JC-F $=37 \mathrm{~Hz}, 2 \mathrm{C}$ ), 57.2, 55.5, 45.2, 44.4, 36.1, 34.5, 33.4, 29.8. FT-IR (thin film, $\mathrm{cm}^{-1}$ ): $3061,3028,2974,1757,1603,1495,1447,1285,1231$, 1170, 1102, 975, 701. HRMS calc'd for $\mathrm{C}_{24} \mathrm{H}_{21} \mathrm{~F}_{6} \mathrm{~N}_{2} \mathrm{O}_{4}\left[\mathrm{M}^{+}\right]$: 501.1375; found: 501.1306 .

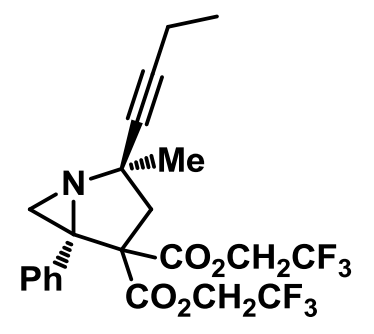

\section{Bis(2,2,2-trifluoroethyl)2-(but-1-ynyl)-2-methyl-5-phenyl-1- azabicyclo[3.1.0] hexane-4,4-dicarboxylate (8k).}

Following experimental procedure C Method A, compound $\mathbf{8 k}$ was prepared by dissolving cyclopropane $2 \mathbf{k}(150 \mathrm{mg}, 0.417 \mathrm{mmol}$ ) and $\operatorname{Dy}(\mathrm{OTf})_{3}(25 \mathrm{mg}, 0.042 \mathrm{mmol})$ in $6 \mathrm{~mL}$ of toluene. (1-azidovinyl)benzene ${ }^{4} 5$ (121 $\mathrm{mg}, 0.833 \mathrm{mmol}$ ), in $1 \mathrm{~mL}$ of toluene, was added dropwise. The reaction was heated at $110{ }^{\circ} \mathrm{C}$ for 18 hours. Compound $8 \mathbf{k}$ (103 mg, 52\%) was obtained as a clear oil. $\mathrm{Rf}=$ 0.44 (30\% EtOAc in hexanes).

Following experimental procedure $\mathrm{C}$ Method B, compound $\mathbf{8 k}$ was prepared by dissolving cyclopropane $\mathbf{2 k}$ (150 mg, $0.417 \mathrm{mmol})$ and $\mathrm{Dy}(\mathrm{OTf})_{3}(25 \mathrm{mg}, 0.042 \mathrm{mmol})$ in $6 \mathrm{~mL}$ of toluene. 3-phenyl-2H-azirine 6 (97 mg, $0.833 \mathrm{mmol}$ ), in $1 \mathrm{~mL}$ of toluene, was added dropwise. The reaction was heated at $110^{\circ} \mathrm{C}$ for 20 hours. Compound 8k (105 mg, 53\%) was obtained as a clear oil.

${ }^{1} \mathrm{H}$ NMR $\left(400 \mathrm{MHz}, \mathrm{CDCl}_{3}\right) \delta=7.54-7.50(\mathrm{~m}, 2 \mathrm{H}), 7.31-7.22(\mathrm{~m}, 3 \mathrm{H}), 4.62(\mathrm{dq}, J=$ 16.4, 8.2 Hz, 1H), 4.50 (qd, $J=16.4,8.2 \mathrm{~Hz}, 1 \mathrm{H}$ ), 4.38 (qd, $J=12.5,8.6 \mathrm{~Hz}, 1 \mathrm{H}$ ), 3.85 (qd, $J=12.5,8.4 \mathrm{~Hz}, 1 \mathrm{H}$ ), $2.98(\mathrm{~d}, J=14.4 \mathrm{~Hz}, 1 \mathrm{H}$ ), 2.31 (d, $J=14.4 \mathrm{~Hz}, 1 \mathrm{H}), 2.25$ (q, $J=7.7 \mathrm{~Hz}, 2 \mathrm{H}), 2.05(\mathrm{~d}, J=1.6 \mathrm{~Hz}, 1 \mathrm{H}), 1.92(\mathrm{~d}, J=1.2 \mathrm{~Hz}, 1 \mathrm{H}), 1.55(\mathrm{~s}, 3 \mathrm{H}) 1.18(\mathrm{t}, J$ $=7.4 \mathrm{~Hz}, 3 \mathrm{H}) .{ }^{19} \mathrm{~F}$ NMR $\left(376 \mathrm{MHz}, \mathrm{CDCl}_{3}\right) \delta=-73.74(\mathrm{t}, J=8.6 \mathrm{~Hz}, 3 \mathrm{~F}),-73.94(\mathrm{t}, J=$ 
8.6 Hz, 3F). ${ }^{13} \mathrm{C}$ NMR $\left(100 \mathrm{MHz}, \mathrm{CDCl}_{3}\right) \delta=168.1,166.4,137.4,128.9,127.6,122.4$ (q, $\left.J_{C-F}=277 \mathrm{~Hz}, 1 \mathrm{C}\right), 122.2\left(q, J_{C-F}=277 \mathrm{~Hz}, 1 \mathrm{C}\right), 85.2,82.6,65.8,61.3\left(q, J_{C-F}=37 \mathrm{~Hz}\right.$, 1C ), 61.2, 61.0 (q, JC-F $=37 \mathrm{~Hz}, 1 \mathrm{C}$ ), 55.7, 45.3, 32.9, 25.8, 13.6, 12.3. FT-IR (thin film, $\left.\mathrm{cm}^{-1}\right)$ : 3062, 3030, 2978, 2939, 2881, 2248, 1759, 1497, 1448, 1411, 1285, 1229, 1170, 1129, 974, 701. HRMS calc'd for $\mathrm{C}_{22} \mathrm{H}_{21} \mathrm{~F}_{6} \mathrm{NO}_{4}\left[\mathrm{M}^{+}\right]$]: 477.1375; found: 477.1303 .

\section{X-Ray Crystallography Data of 8b (CDCD 1486123)}

X-ray quality crystals were prepared by vapor diffusion of cyclohexane into a solution of $\mathbf{8 b}$ in minimal dichloromethane. All X-ray measurements were made on a Bruker Kappa Axis Apex2 diffractometer at a temperature of $110 \mathrm{~K}$.

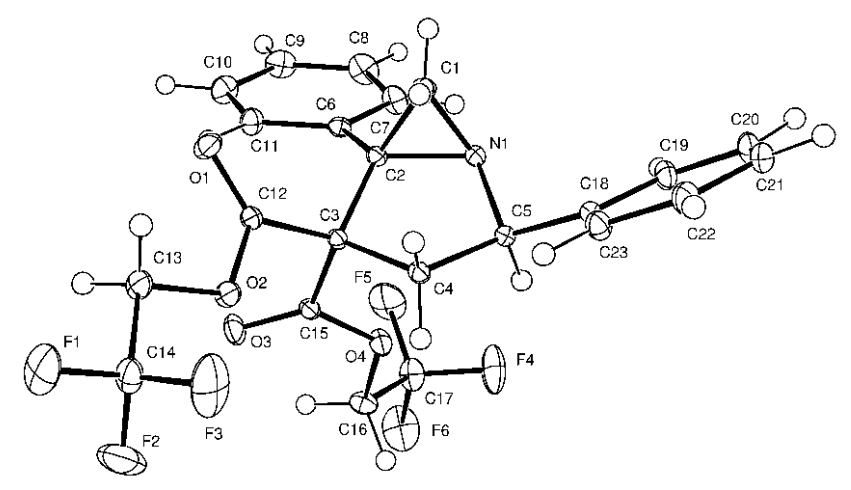

Table S1. Summary of Crystal Data for $8 b$

Formula

Formula Weight $(\mathrm{g} / \mathrm{mol})$

Crystal Dimensions $(\mathrm{mm}$ )

Crystal Color and Habit

Crystal System

Space Group

Temperature, $\mathrm{K}$
$\mathrm{C}_{23} \mathrm{H}_{19} \mathrm{~F}_{6} \mathrm{NO}_{4}$

487.39

$0.429 \times 0.326 \times 0.270$

colourless prism

triclinic

P -1

110 
$a, \AA$

$b, \AA$

$c, \AA$

$\alpha,{ }^{\circ}$

$\beta, \circ$

$\gamma,{ }^{\circ}$

$\mathrm{V}, \AA^{3}$

Number of reflections to determine final unit cell

Min and Max $2 \theta$ for cell determination, ${ }^{\circ}$

$\mathrm{Z}$

$\mathrm{F}(000)$

$\rho(\mathrm{g} / \mathrm{cm})$

$\lambda, \AA$, (MoK $\alpha)$

$\mu,\left(\mathrm{cm}^{-1}\right)$

Diffractometer Type

Scan Type(s)

Max $2 \theta$ for data collection, ${ }^{\circ}$

Measured fraction of data

Number of reflections measured

Unique reflections measured

$\mathrm{R}_{\text {merge }}$

Number of reflections included in refinement

Cut off Threshold Expression

Structure refined using

Weighting Scheme

Number of parameters in least-squares

$\mathrm{R}_{1}$

$\mathrm{wR}_{2}$

$\mathrm{R}_{1}$ (all data)

$w_{2}$ (all data)

GOF

Maximum shift/error
8.5114(17)

9.495(2)

13.368(4)

98.002(14)

95.439(9)

96.685(9)

1055.7(5)

9127

$4.84,88.34$

2

500

1.533

0.71073

0.139

Bruker Kappa Axis Apex2

phi and omega scans

89.118

0.998

85998

15039

0.0268

15039

I > $2 \operatorname{sigma}(\mathrm{I})$

full matrix least-squares using $\mathrm{F}^{2}$

$\mathrm{w}=1 /\left[\operatorname{sigma}^{2}\left(\mathrm{Fo}^{2}\right)+(0.0645 \mathrm{P})^{2}+0.1378\right.$

$\mathrm{P}$ ] where $\mathrm{P}=\left(\mathrm{Fo}^{2}+2 \mathrm{Fc}^{2}\right) / 3$

383

0.0398

0.1118

0.0531

0.1202

1.035

0.001 


\section{X-Ray Crystallography Data of 8g (CDCD 1486124)}

X-ray quality crystals were prepared by vapor diffusion of cyclohexane into a solution of $\mathbf{8 g}$ in minimal dichloromethane. All X-ray measurements were made on a Bruker Kappa Axis Apex2 diffractometer at a temperature of $110 \mathrm{~K}$.

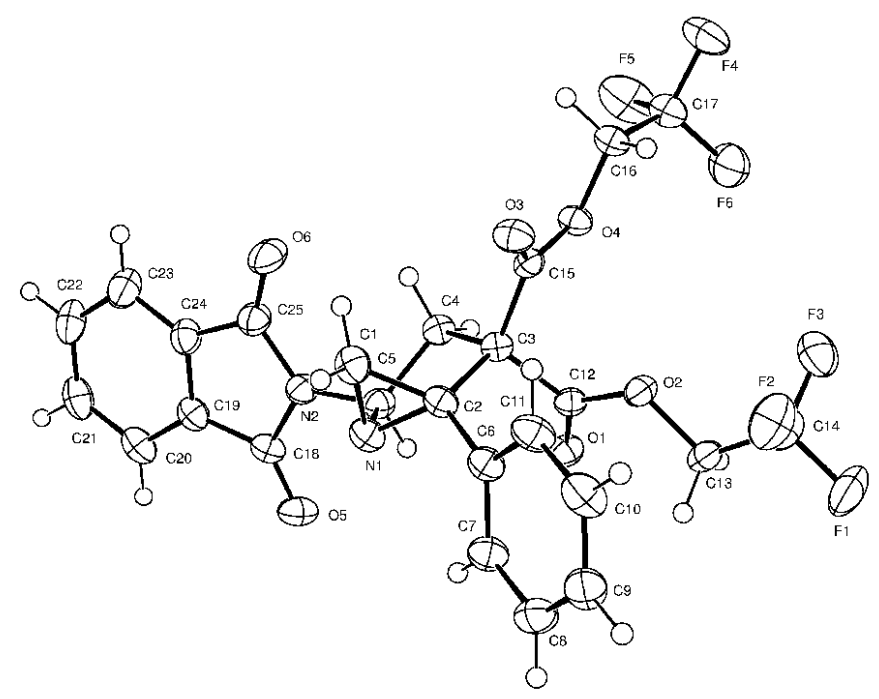

Table 1. Summary of Crystal Data for $n 16035$

Formula

Formula Weight $(\mathrm{g} / \mathrm{mol})$

Crystal Dimensions $(\mathrm{mm}$ )

Crystal Color and Habit

Crystal System

Space Group
$\mathrm{C}_{25} \mathrm{H}_{18} \mathrm{~F}_{6} \mathrm{~N}_{2} \mathrm{O}_{6}$

556.41

$0.218 \times 0.140 \times 0.102$

colourless prism

triclinic

P -1 
Temperature, $\mathrm{K}$

$a, \AA$

$b, \AA$

$c, \AA$

$\alpha,^{\circ}$

$\beta,^{\circ}$

$\gamma$,

$\mathrm{V}, \AA^{3}$

Number of reflections to determine final unit cell

Min and Max $2 \theta$ for cell determination, ${ }^{\circ}$

Z

$\mathrm{F}(000)$

$\rho(\mathrm{g} / \mathrm{cm})$

$\lambda, \AA,(\mathrm{CuK} \alpha)$

$\mu,\left(\mathrm{cm}^{-1}\right)$

Diffractometer Type

Scan Type(s)

$\operatorname{Max} 2 \theta$ for data collection, ${ }^{\circ}$

Measured fraction of data

Number of reflections measured

Unique reflections measured

$\mathrm{R}_{\text {merge }}$

Number of reflections included in refinement

Cut off Threshold Expression

Structure refined using

Weighting Scheme

Number of parameters in least-squares

$\mathrm{R}_{1}$

$\mathrm{wR}_{2}$

$\mathrm{R}_{1}$ (all data)

$\mathrm{wR}_{2}$ (all data)

GOF
110

9.2060(18)

11.1007(16)

$13.748(2)$

$70.276(7)$

$77.582(7)$

67.549(8)

1216.3(4)

6519

$8.98,133.14$

2

568

1.519

1.54178

1.220

Bruker-Nonius KappCCD Apex2

phi and omega scans

133.132

0.962

15243

4132

0.0310

4132

I > $2 \operatorname{sigma}(\mathrm{I})$

full matrix least-squares using $\mathrm{F}^{2}$

$\mathrm{w}=1 /\left[\operatorname{sigma}^{2}\left(\mathrm{Fo}^{2}\right)+(0.0576 \mathrm{P})^{2}+0.4707\right.$

$\mathrm{P}]$ where $\mathrm{P}=\left(\mathrm{Fo}^{2}+2 \mathrm{Fc}^{2}\right) / 3$

424

0.0384

0.1009

0.0445

0.1062

1.050 
Maximum shift/error

0.000

Min \& Max peak heights on final $\Delta \mathrm{F} \operatorname{Map}\left(e^{-} / \AA ̊ \AA\right)$

$-0.432,0.572$

Where:

$\mathrm{R}_{1}=\Sigma\left(\left|\mathrm{F}_{\mathrm{o}}\right|-\left|\mathrm{F}_{\mathrm{c}}\right|\right) / \Sigma \mathrm{F}_{\mathrm{o}}$

$\mathrm{wR}_{2}=\left[\Sigma\left(w\left(\mathrm{~F}_{\mathrm{o}}^{2}-\mathrm{F}_{\mathrm{c}}^{2}\right)^{2}\right) / \Sigma\left(w \mathrm{~F}_{\mathrm{o}}^{4}\right)\right]^{1 / 2}$

$\mathrm{GOF}=\left[\Sigma\left(w\left(\mathrm{~F}_{\mathrm{o}}{ }^{2}-\mathrm{F}_{\mathrm{c}}{ }^{2}\right)^{2}\right) /(\text { No. of reflns. - No. of params. })\right]^{1 / 2}$

\section{References:}

1. Takacs, J.M; Xu, Z.; Jiang, X-T.; Leonov, A.P.; Theriot, G.C. Org. Lett. 2002, 4, 3843-3845.

2. de Nanteuil, F.; Loup, J.; Waser, J. Org. Lett. 2013, 15, 3738-3741.

3. Armstrong, E. L.; Kerr, M.A. Org. Chem. Front. 2015, 2, 1045-1047.

4. Loy, N.S.Y.; Kim, S.; Park, C-M. Org. Lett. 2015, 17, 395-397. 


\section{${ }^{1} \mathrm{H}$ NMR, ${ }^{19} \mathrm{~F}$ NMR and ${ }^{13} \mathrm{C}$ NMR Spectra}




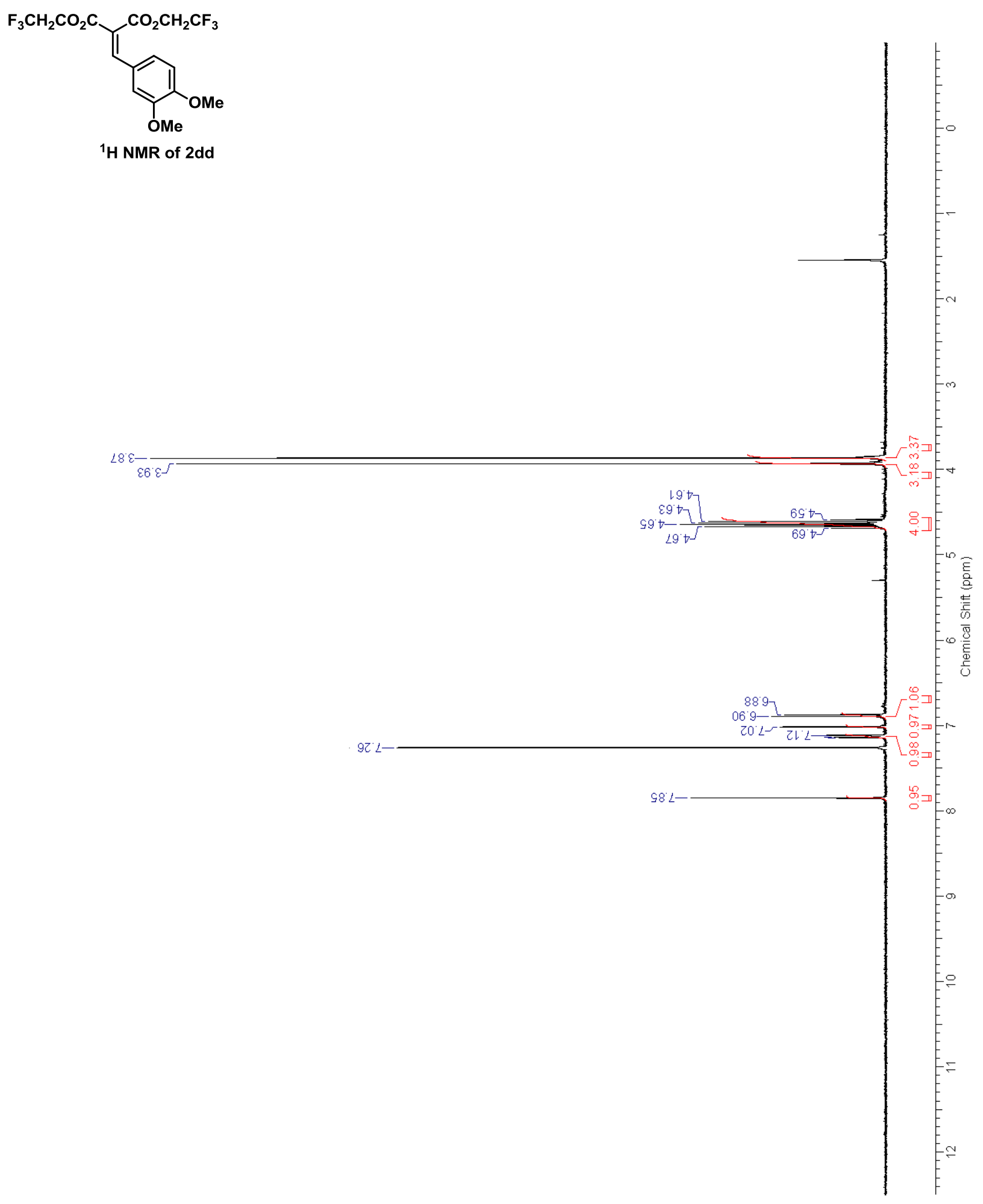



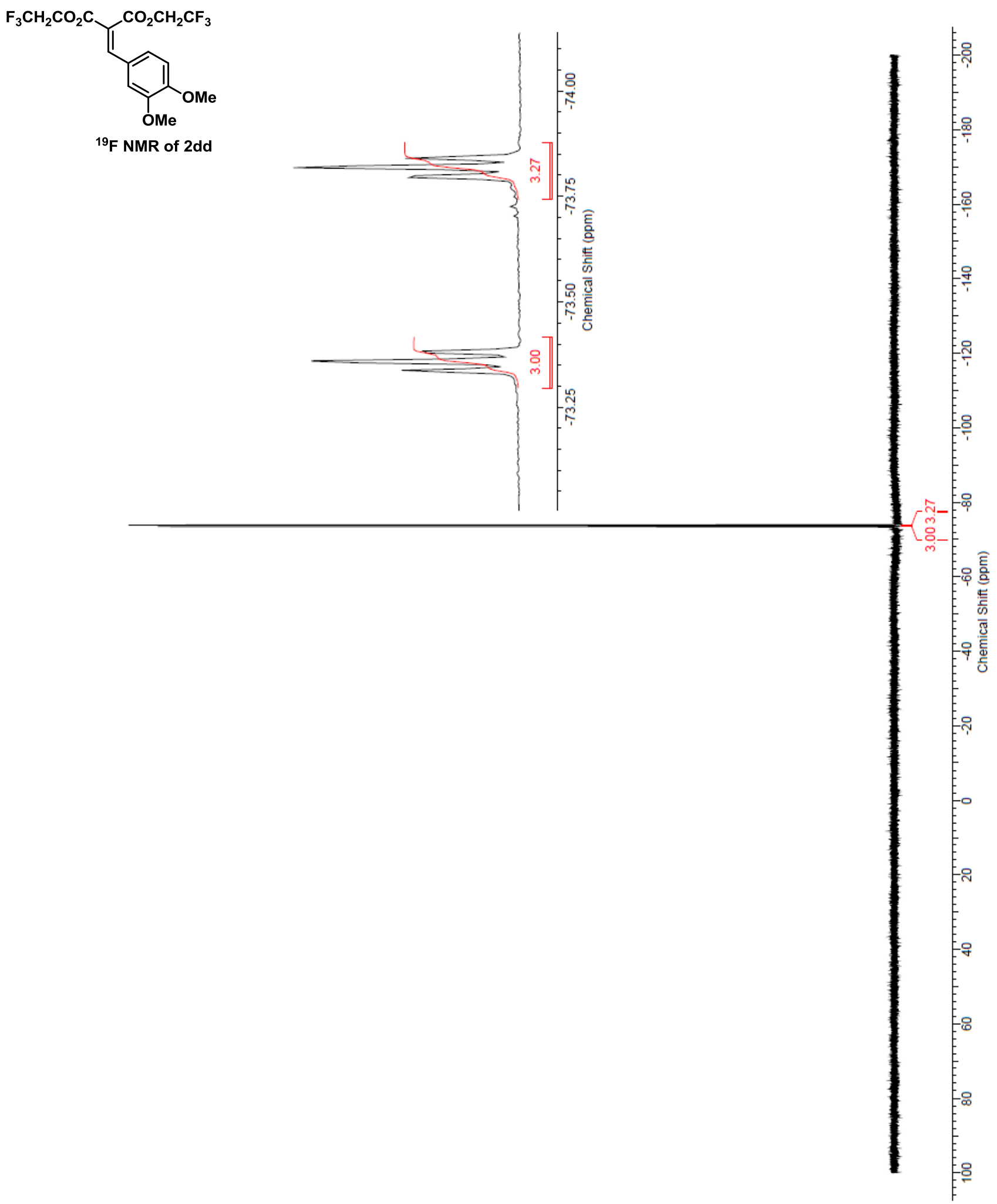


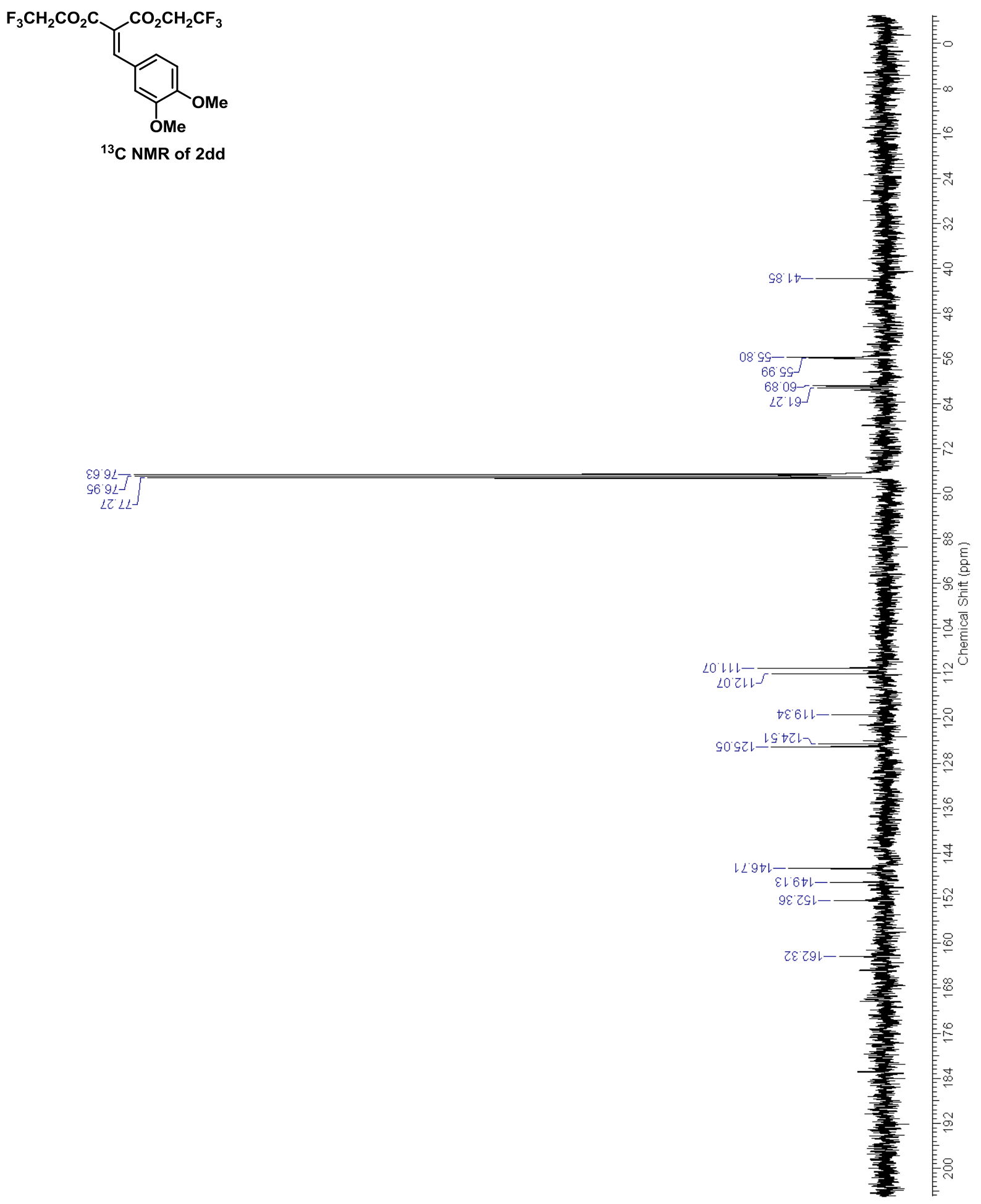



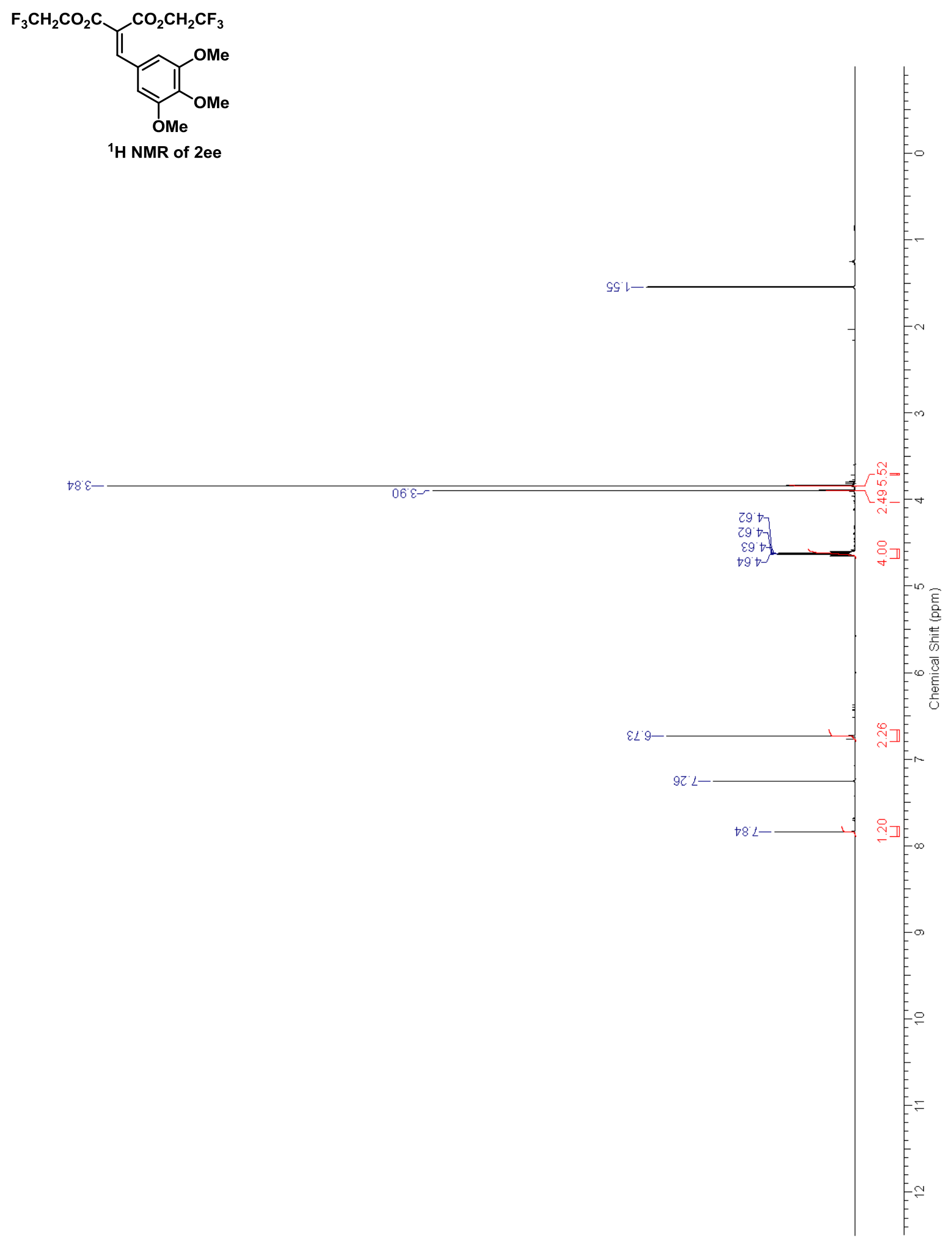


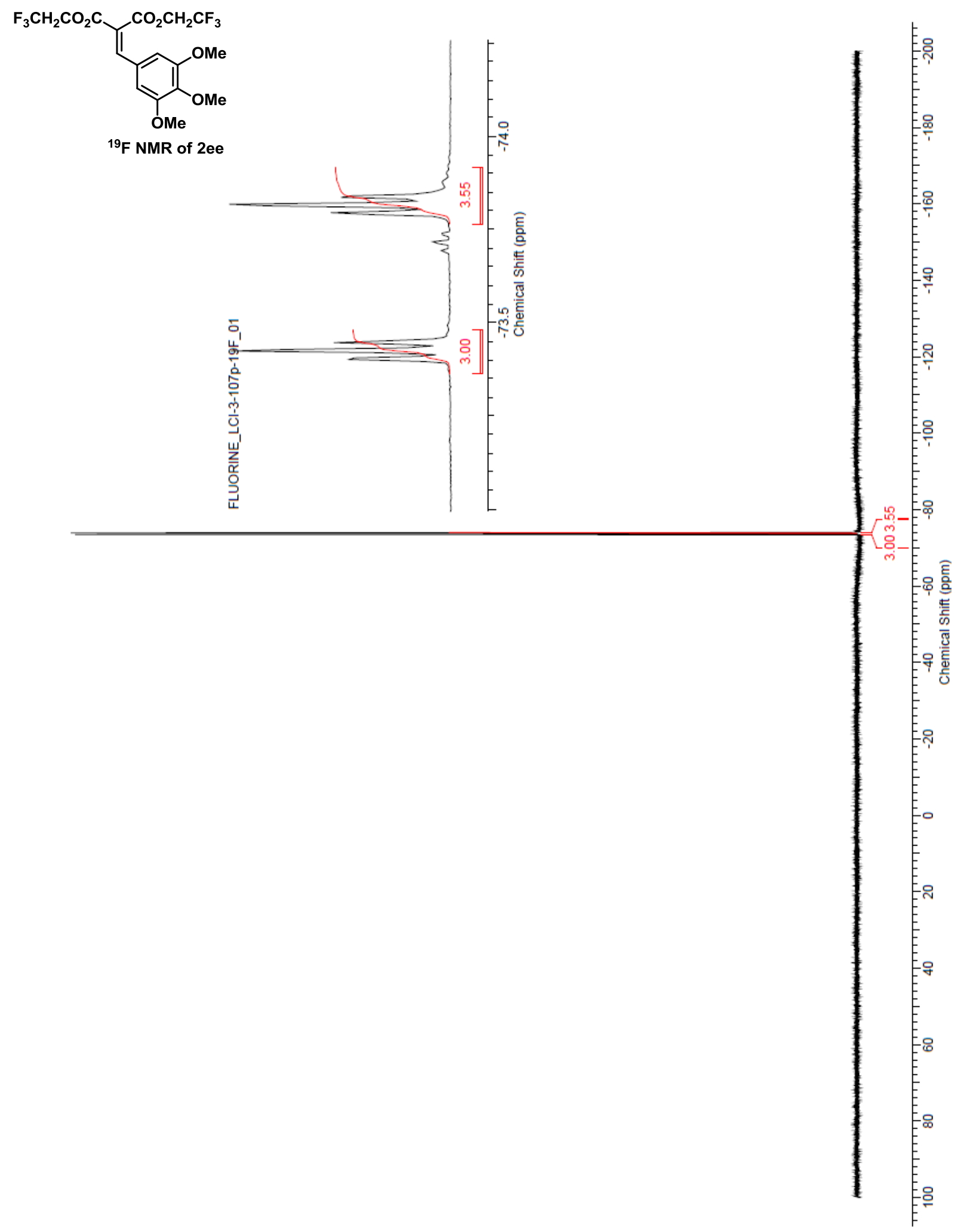




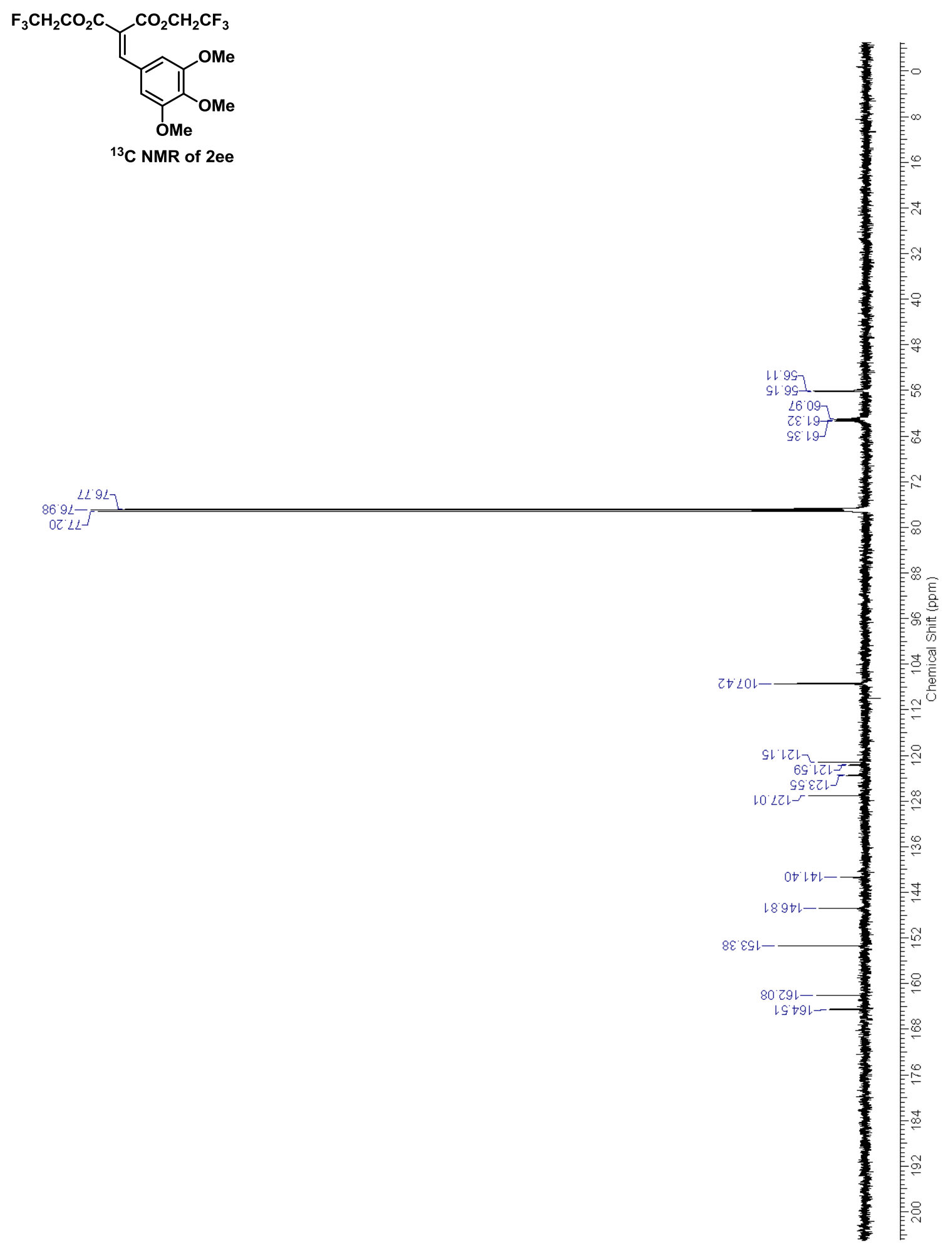




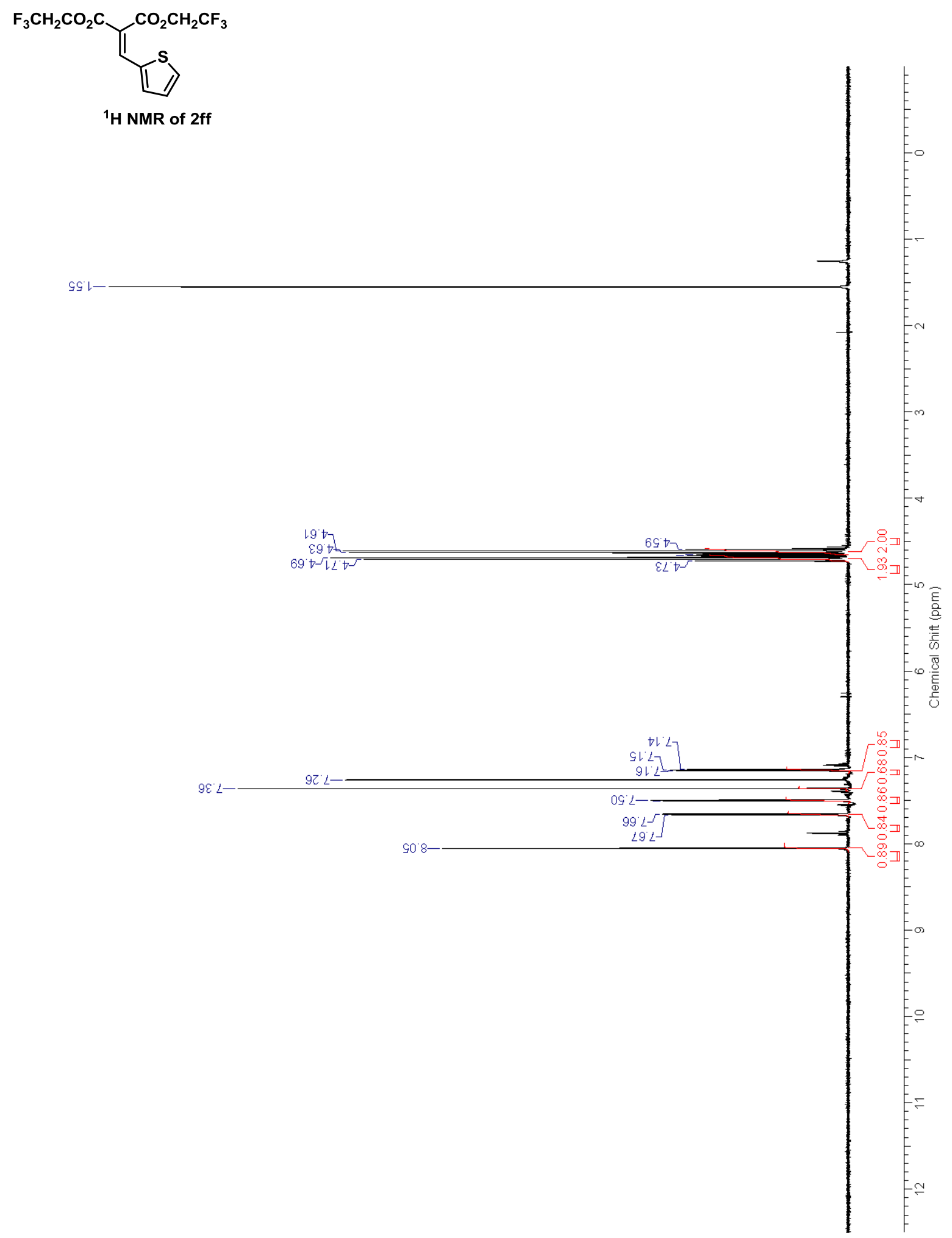



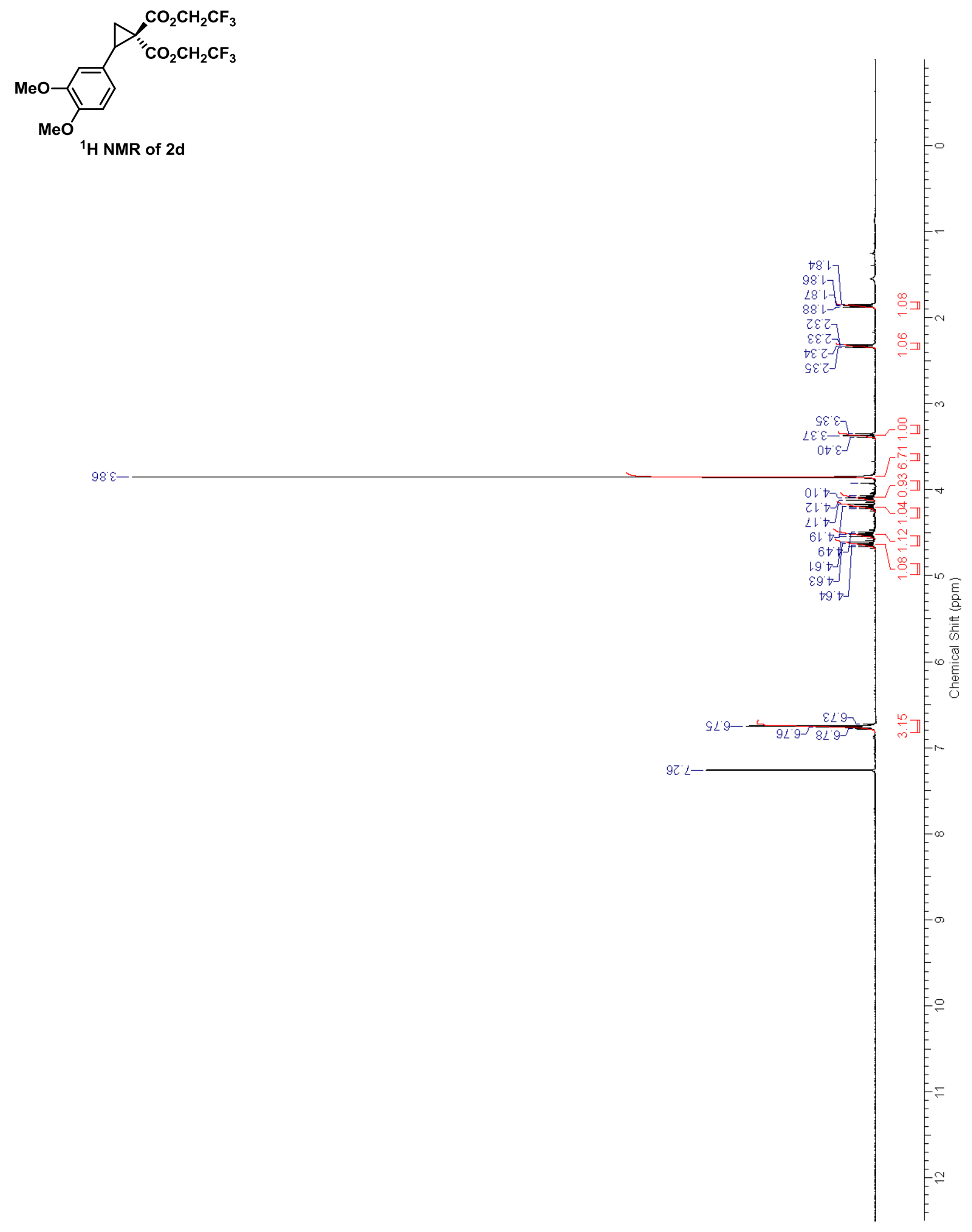

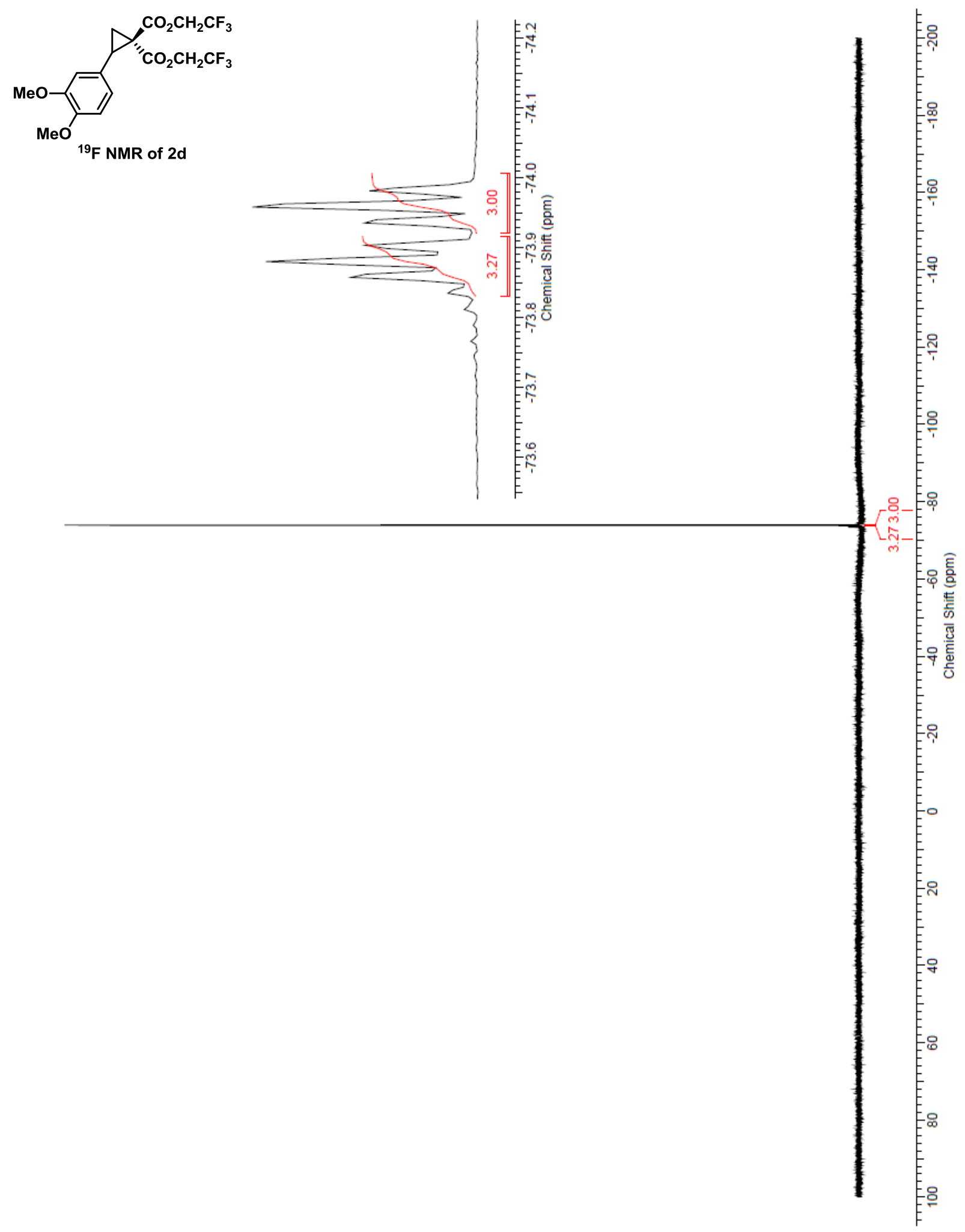

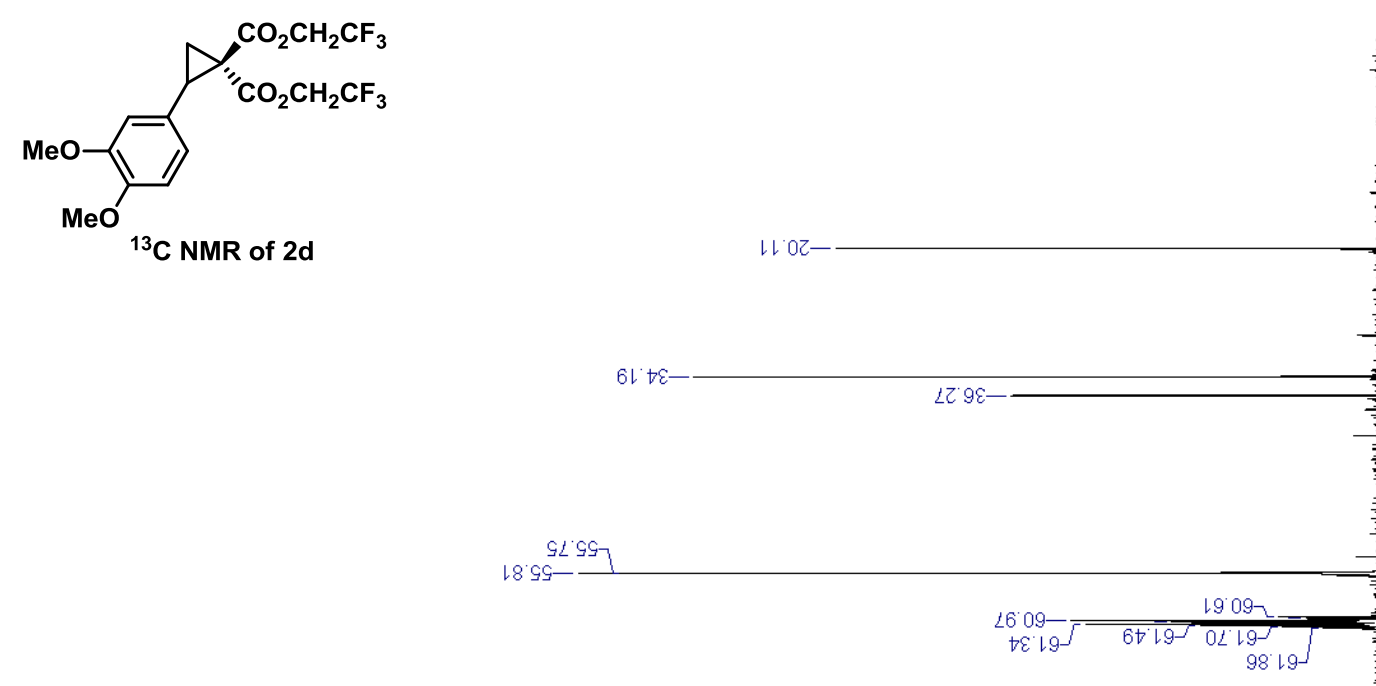

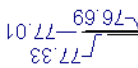

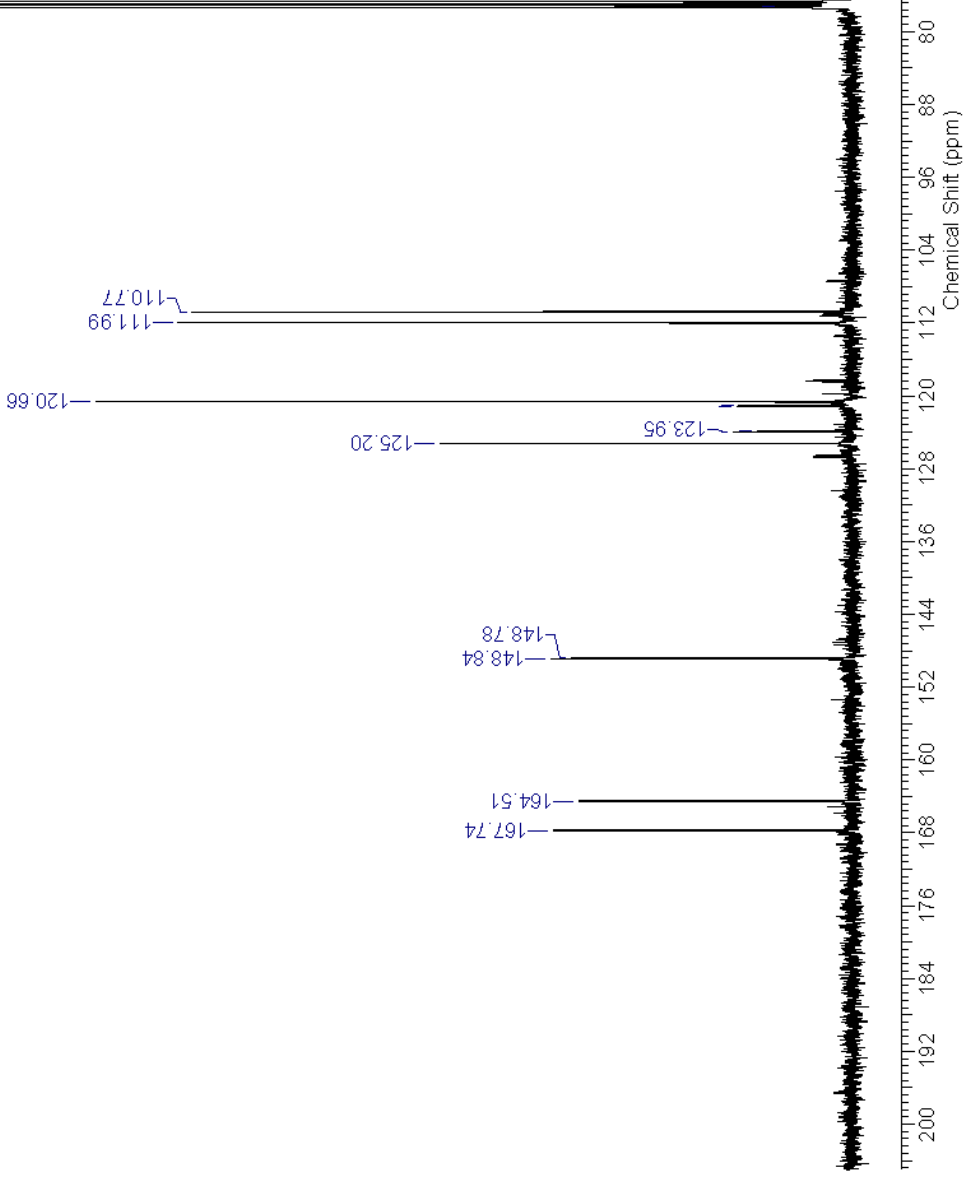



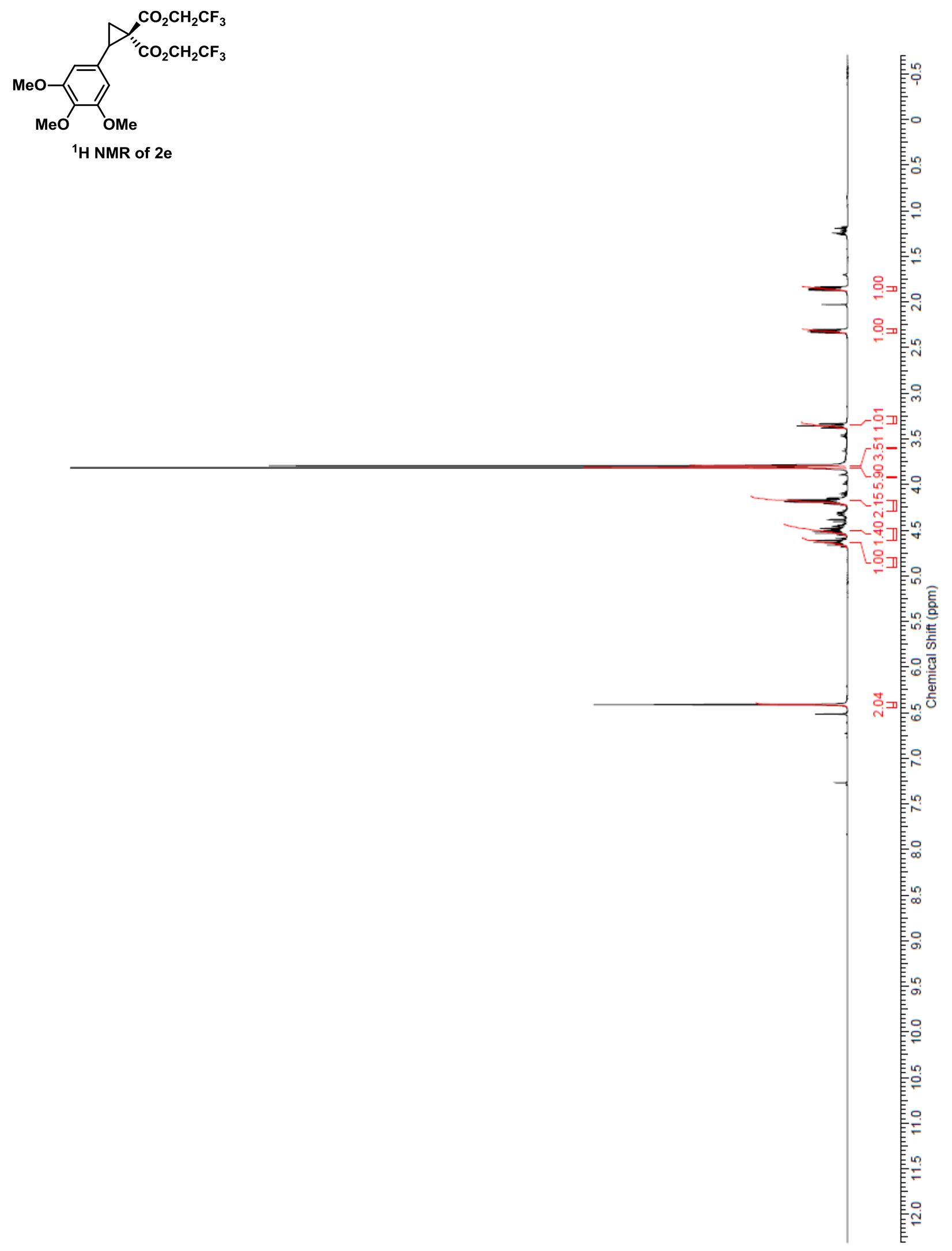

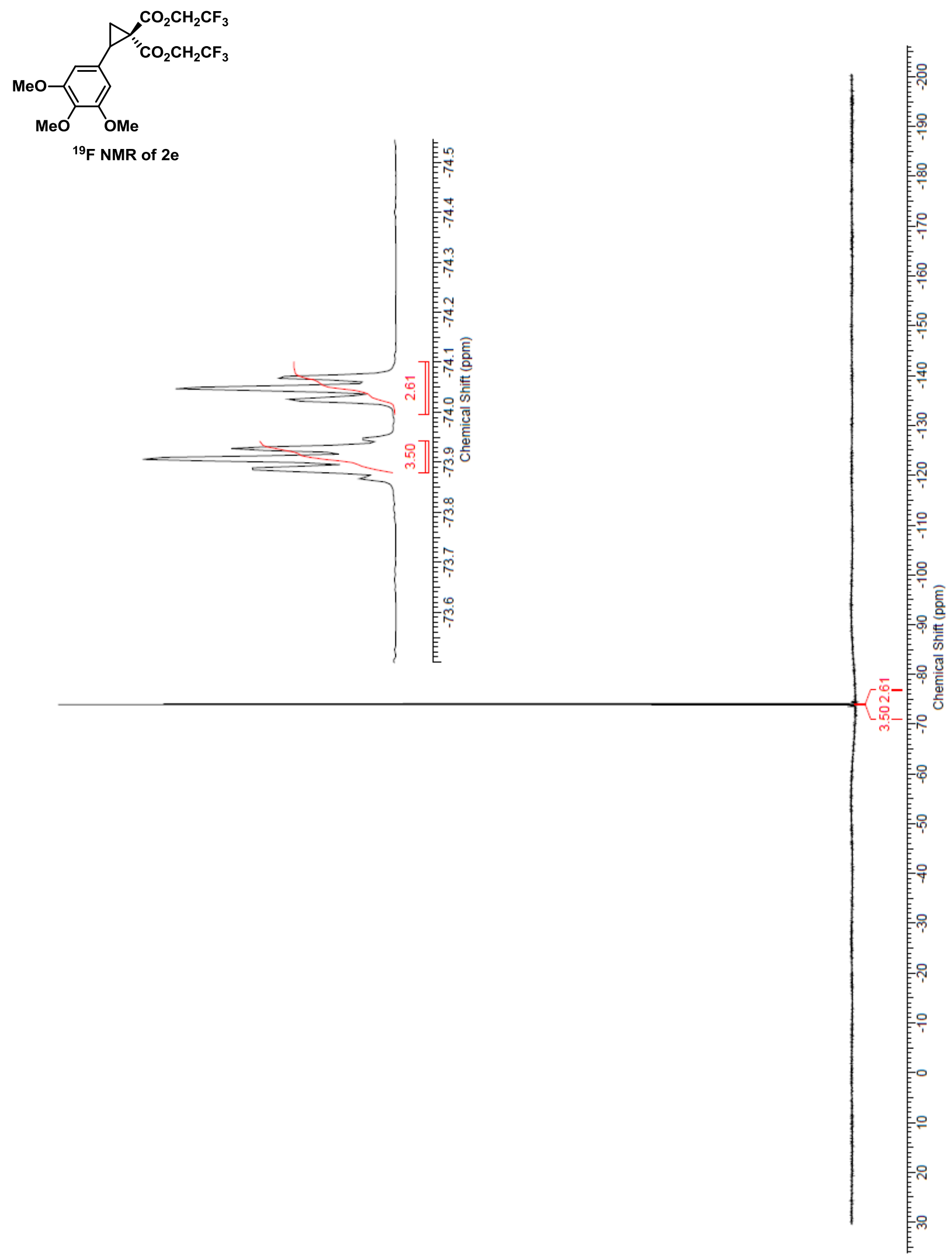

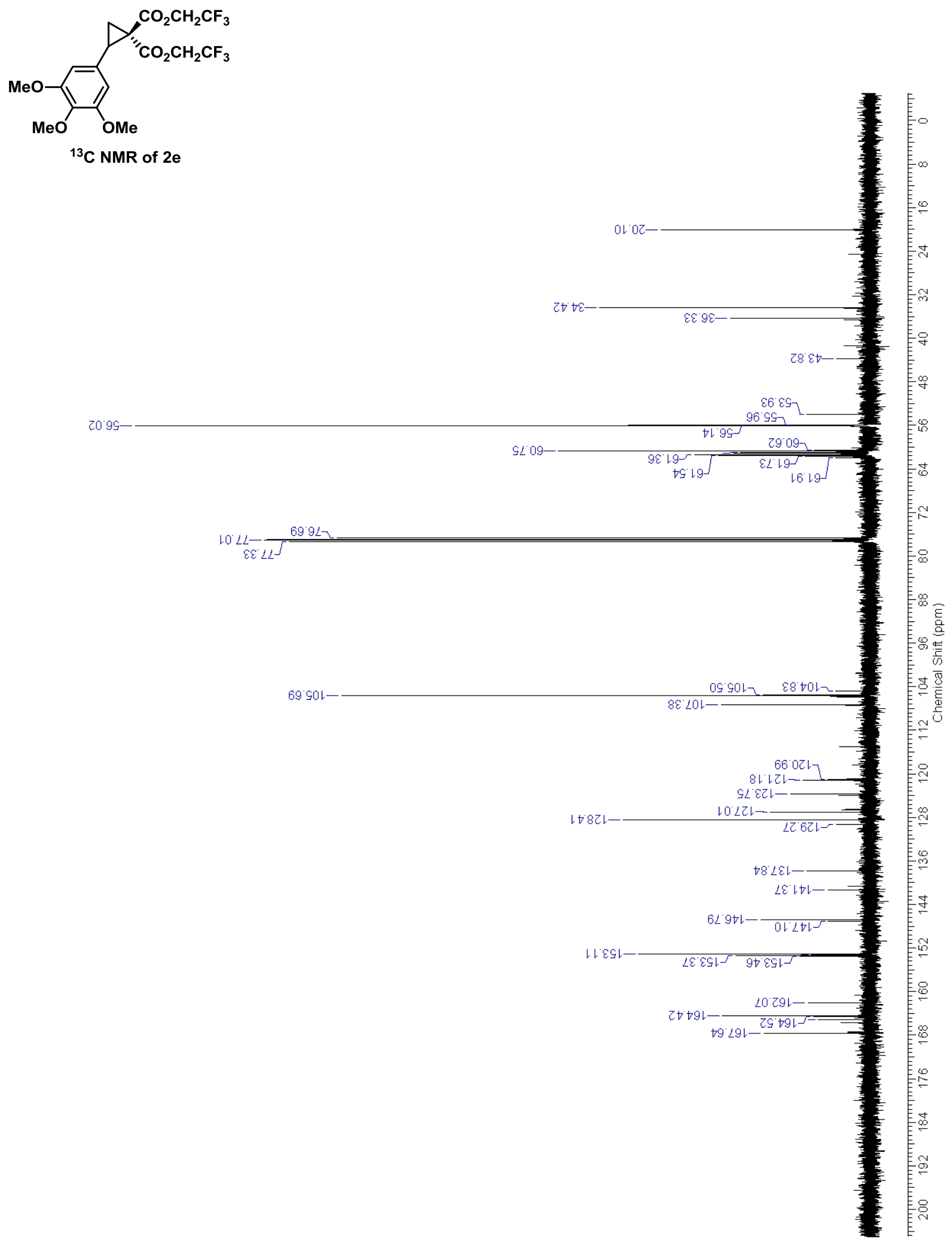

37 

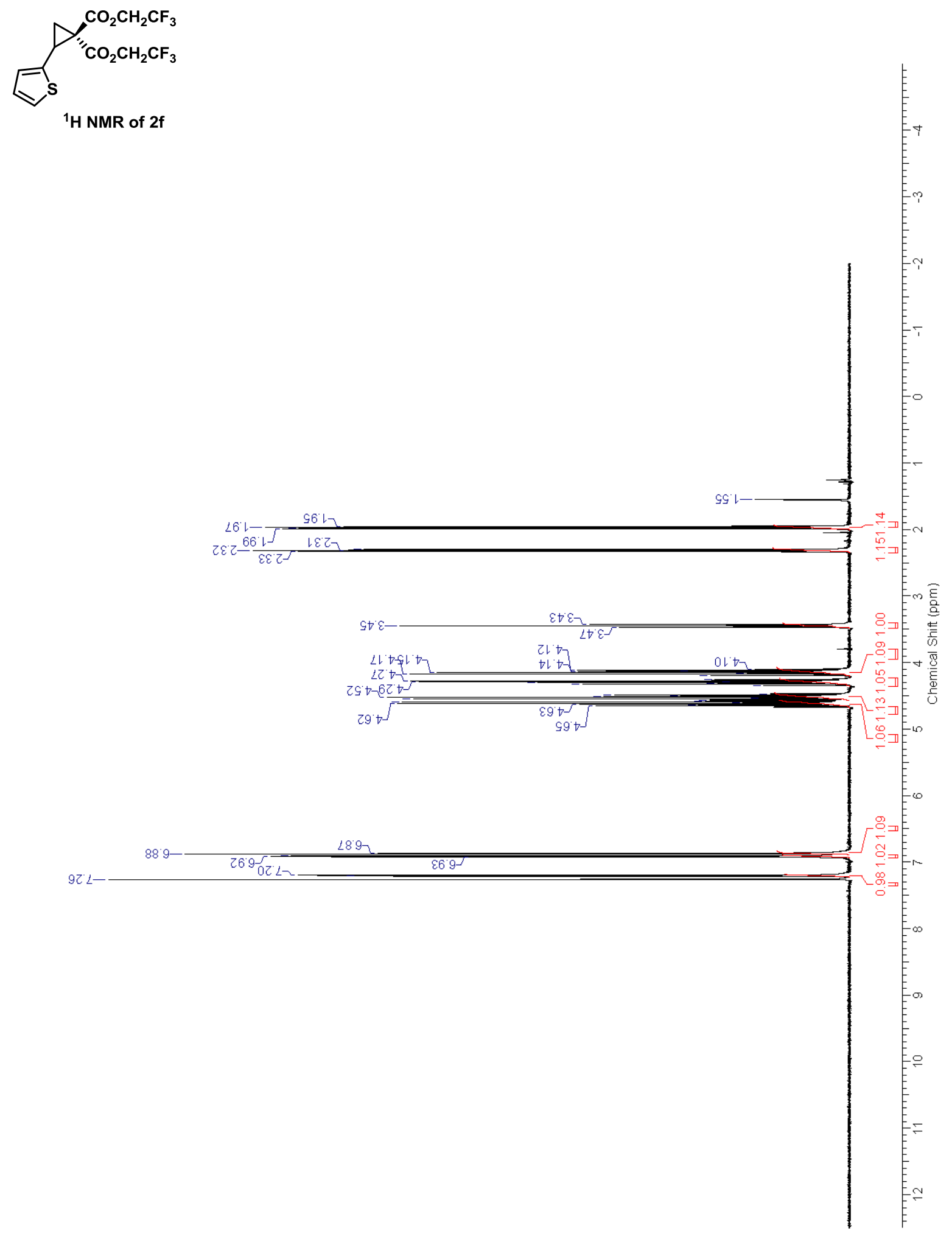

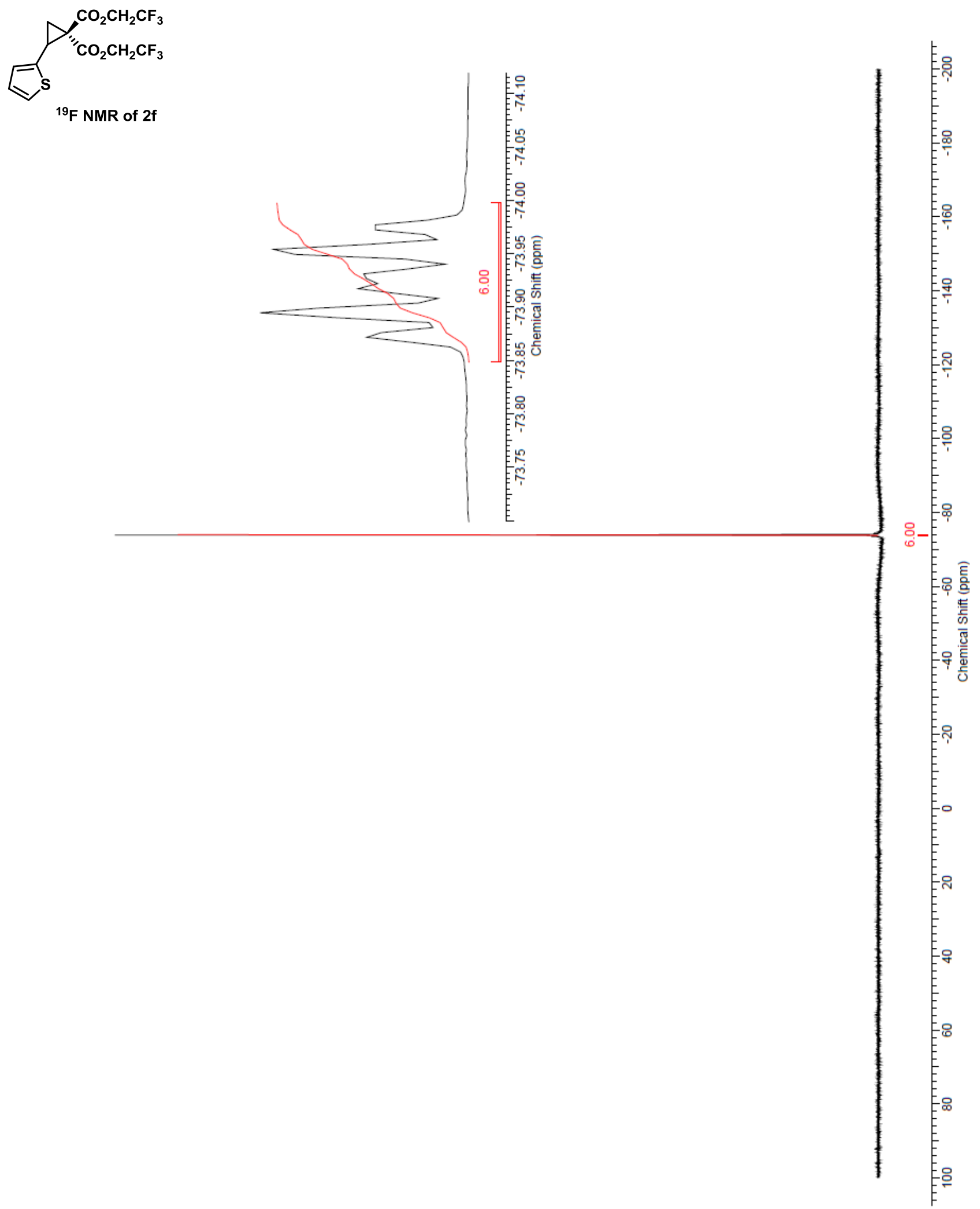

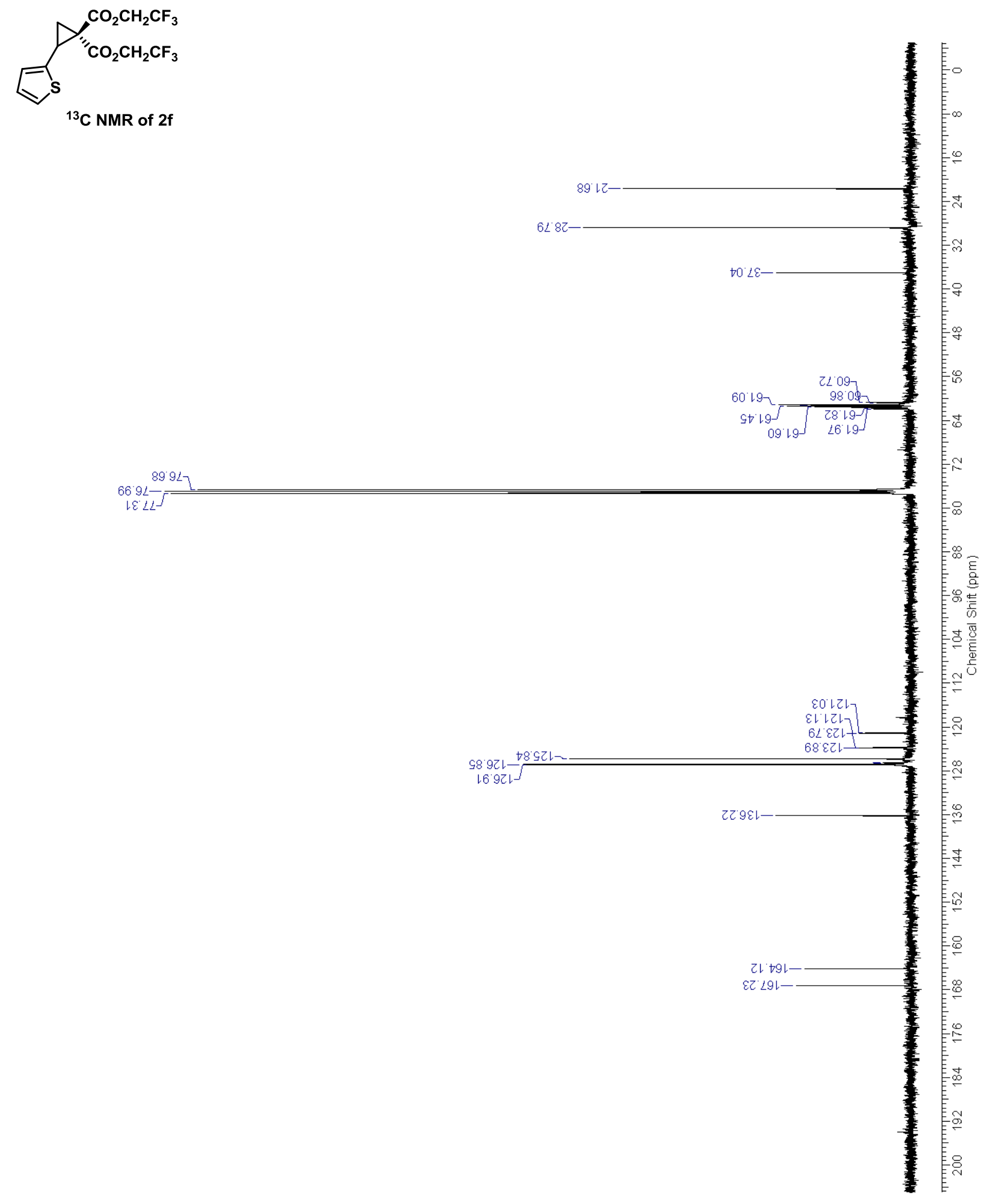


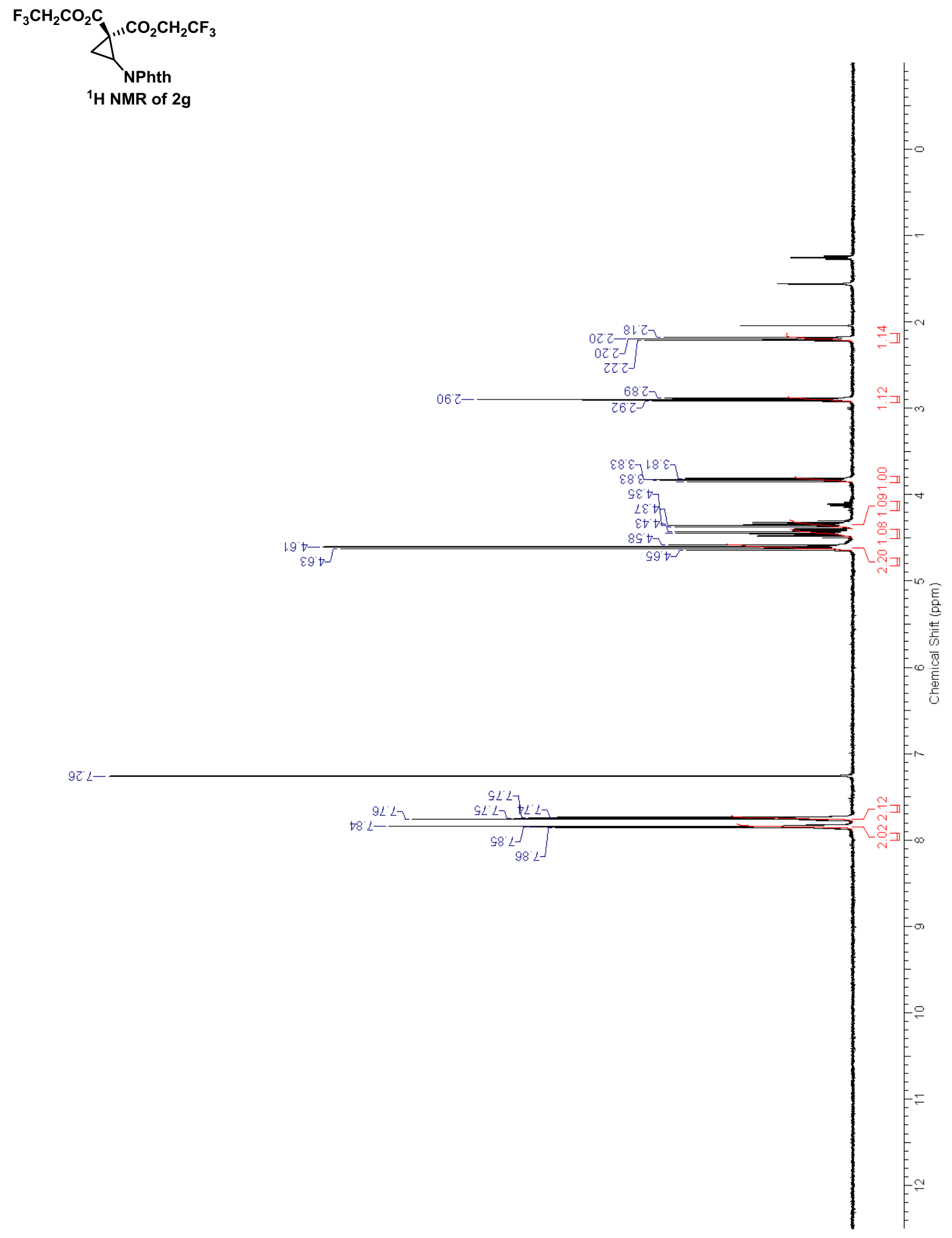



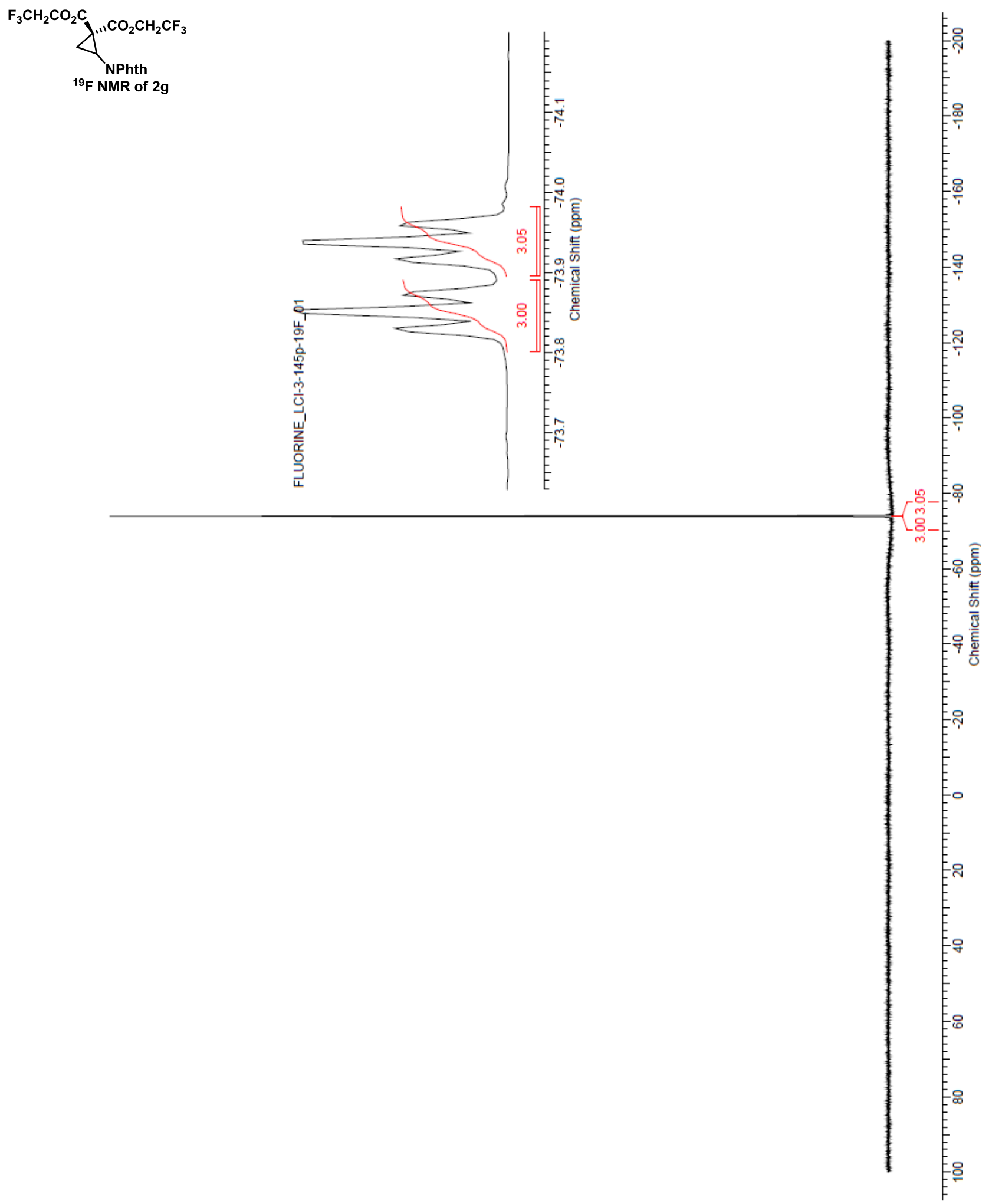

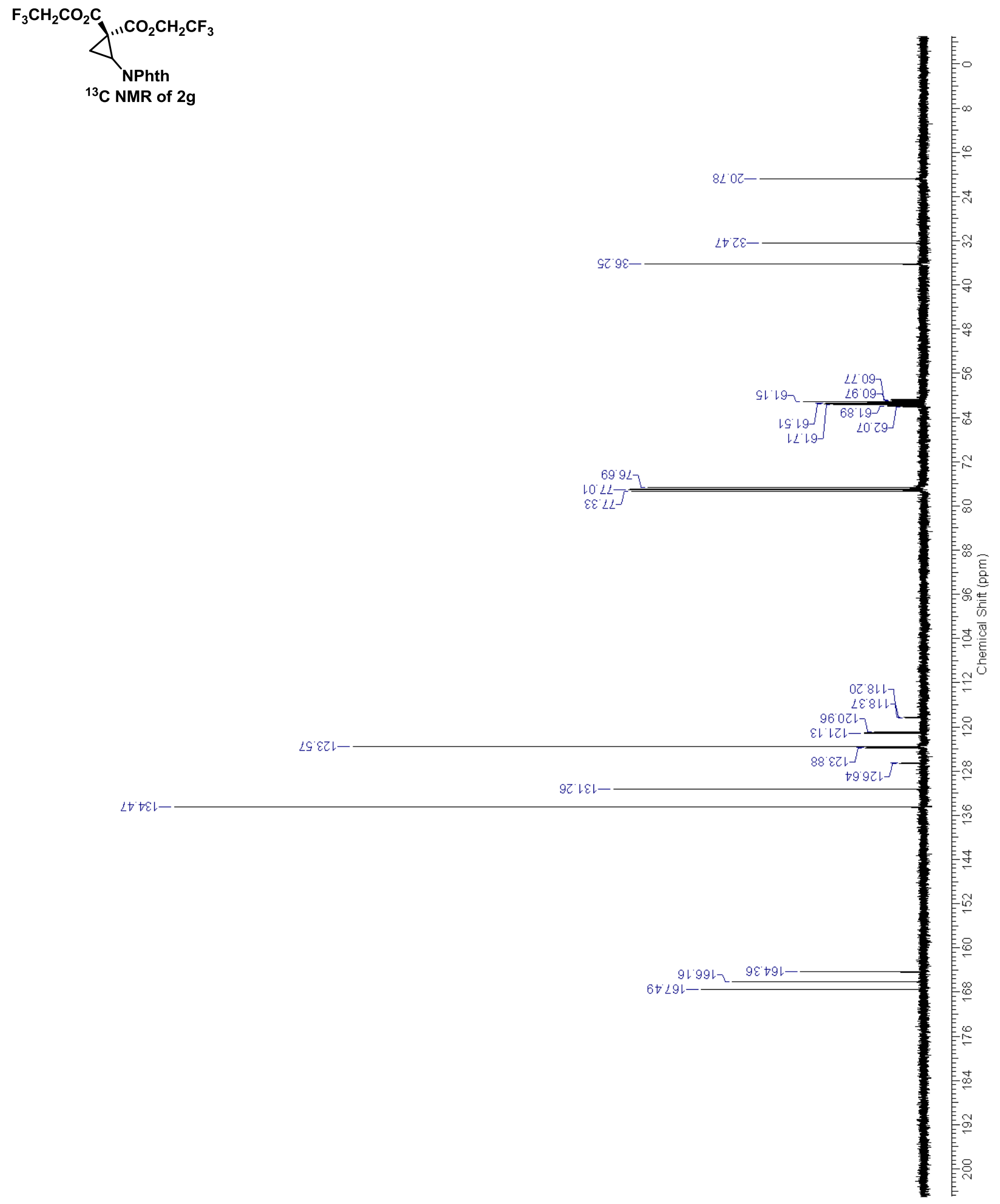


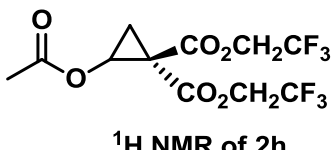

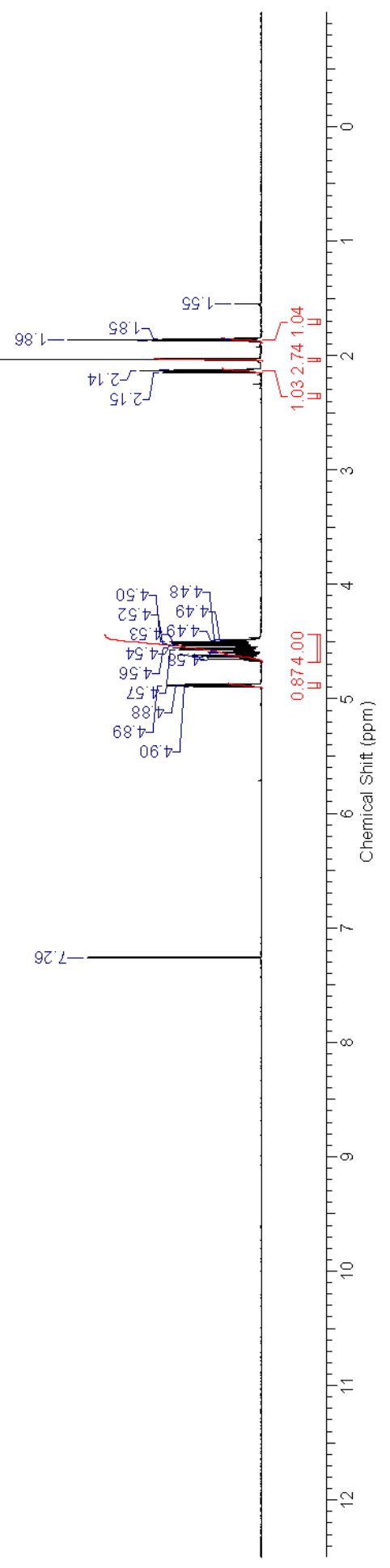



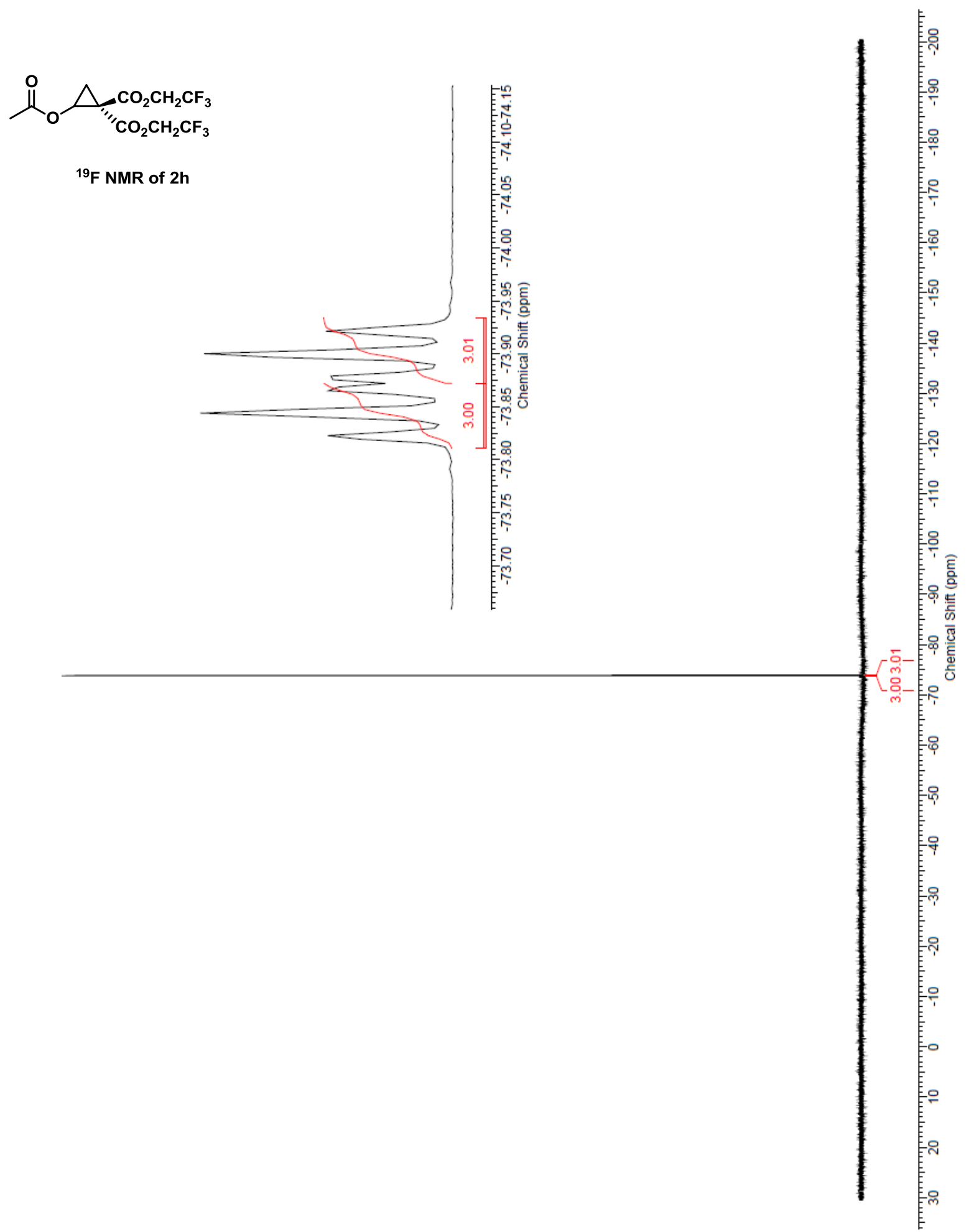

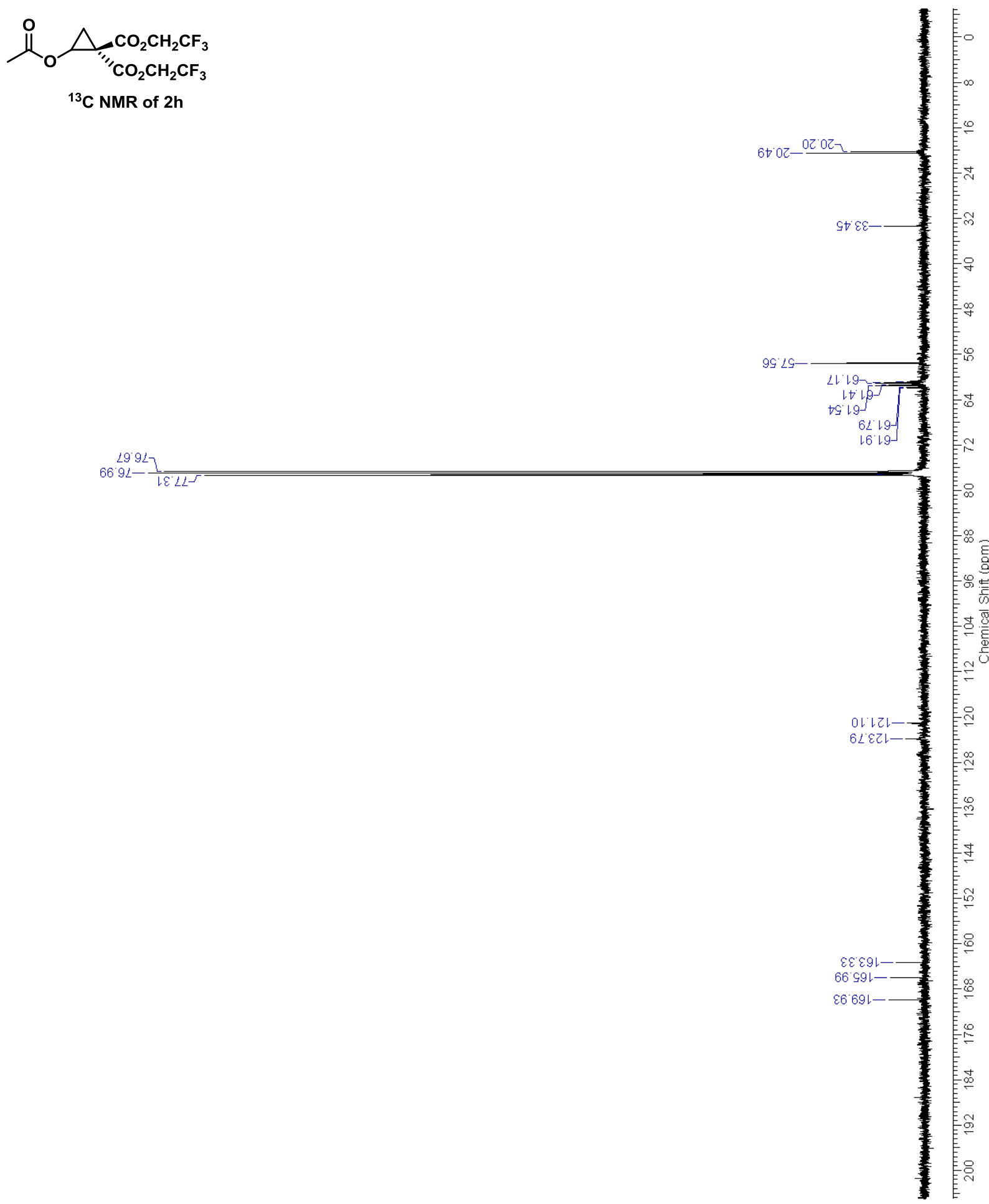


\section{$\underset{\mathrm{Me}}{\mathrm{Ph}} \bigwedge^{\prime \prime} \mathrm{CO}_{2} \mathrm{CH}_{2} \mathrm{CH}_{2} \mathrm{CF}_{3}$ \\ ${ }^{1} \mathrm{H}$ NMR of $2 \mathrm{j}$}

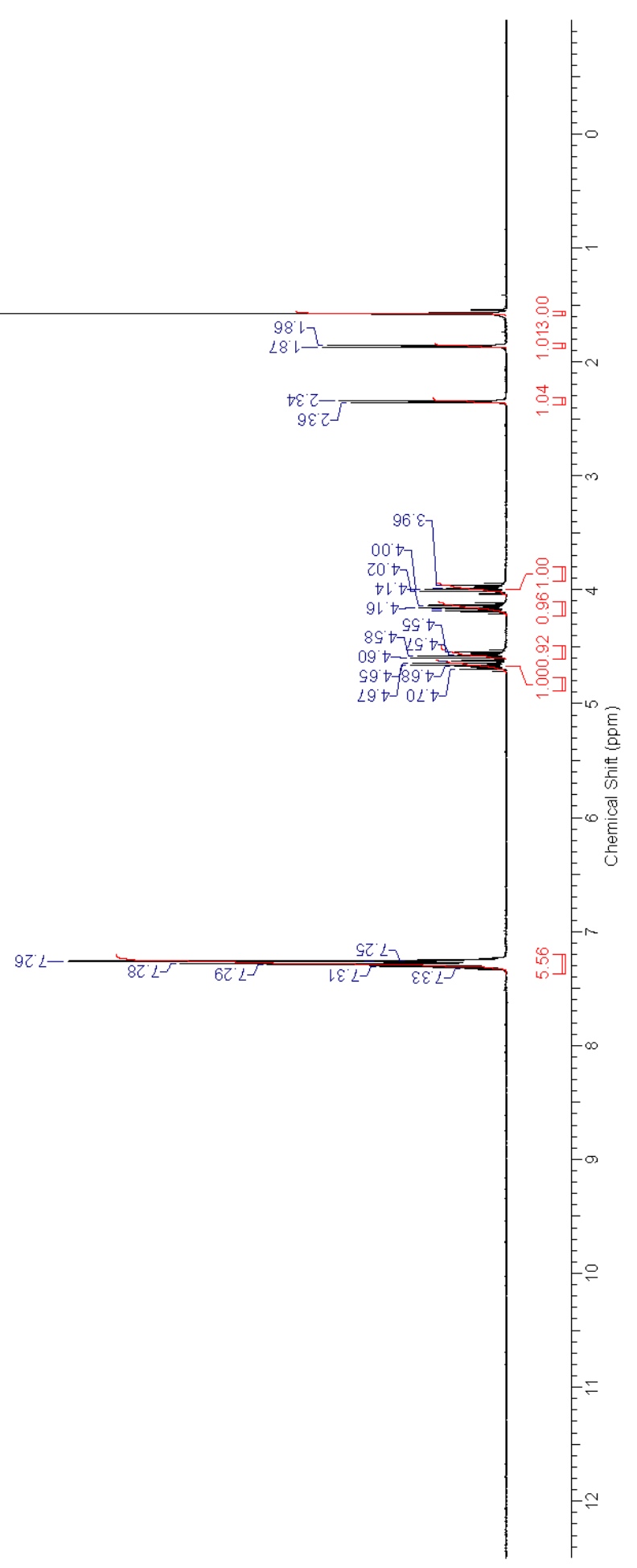



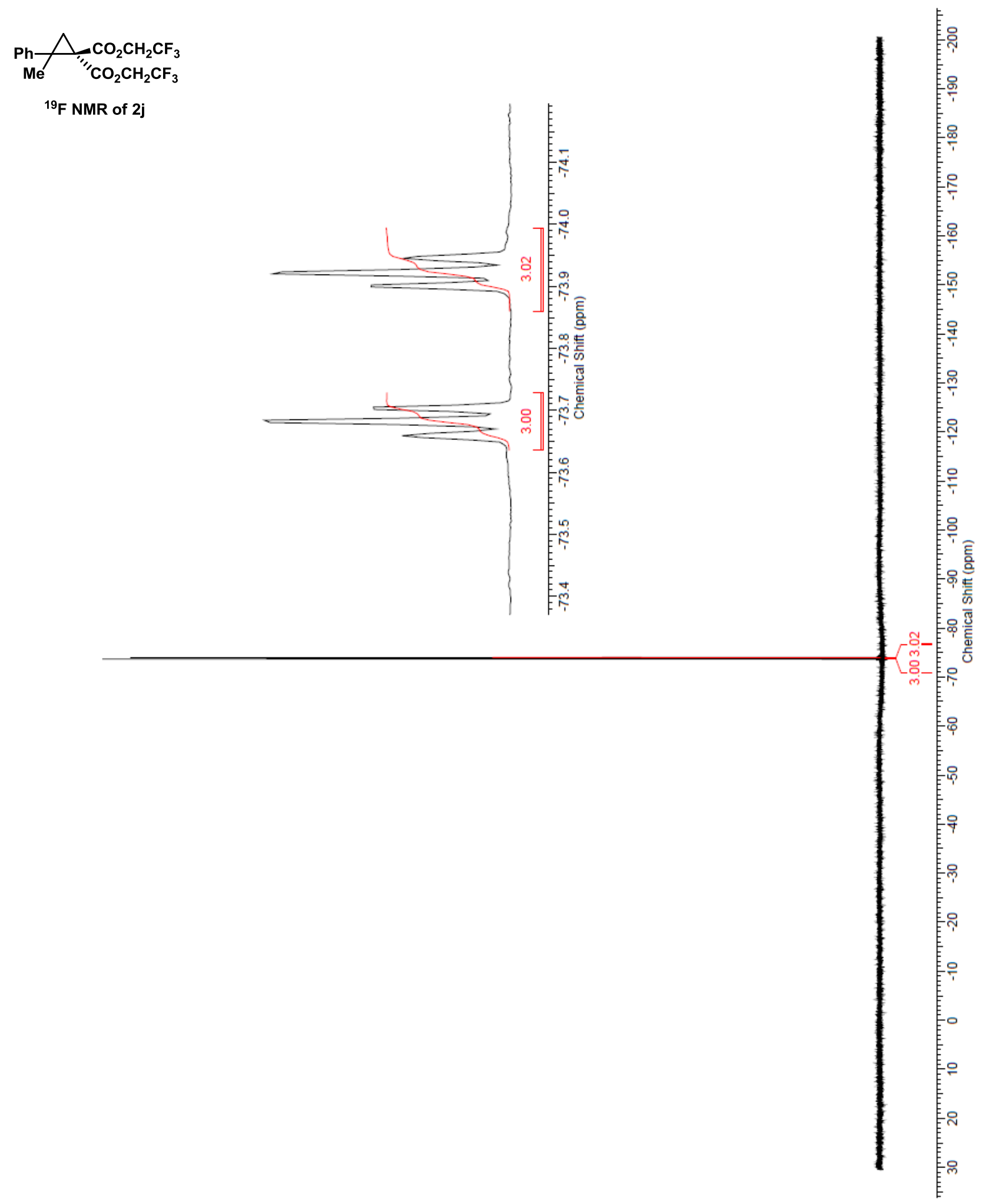


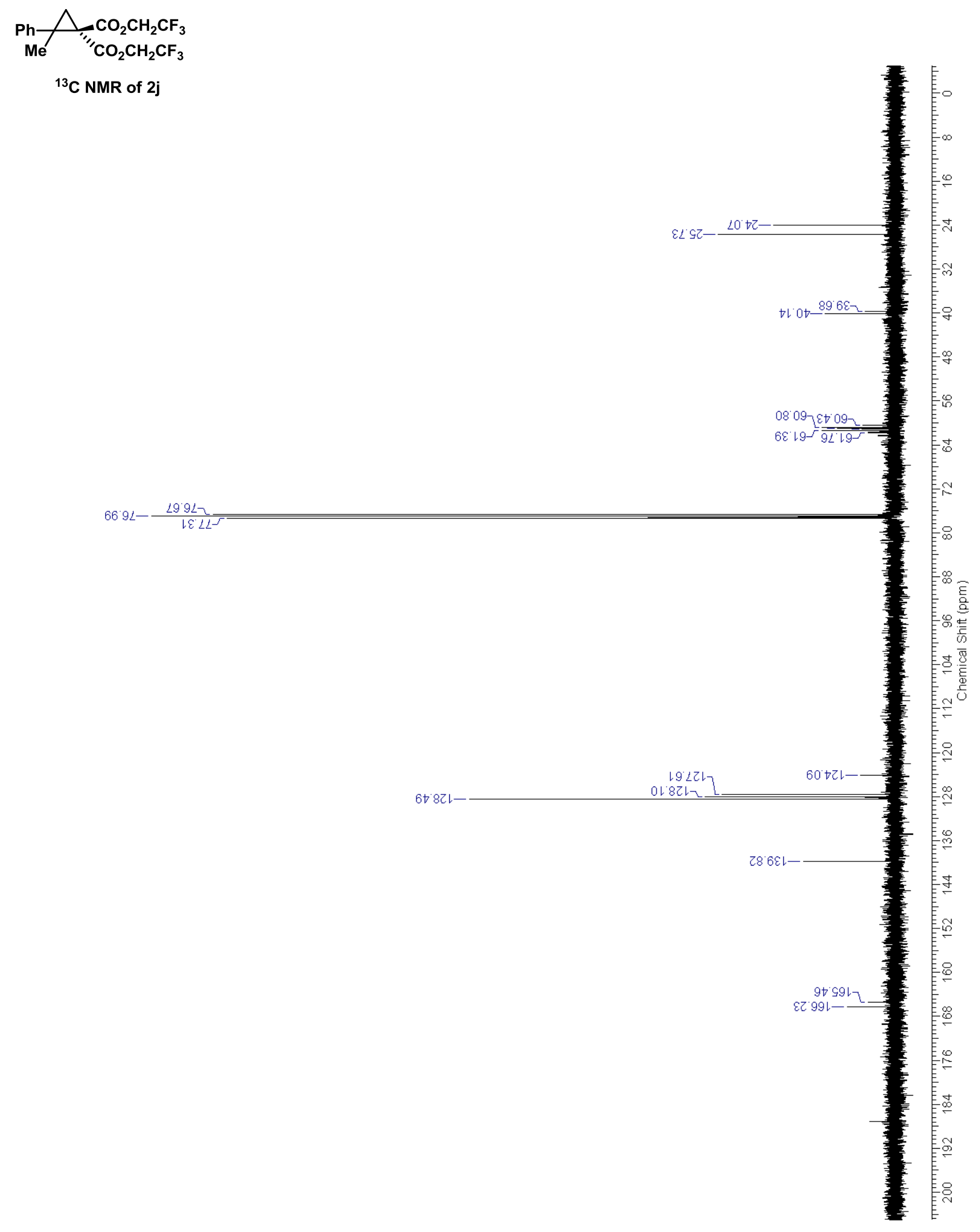




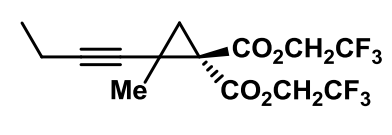

${ }^{1} \mathrm{H}$ NMR of $2 k$

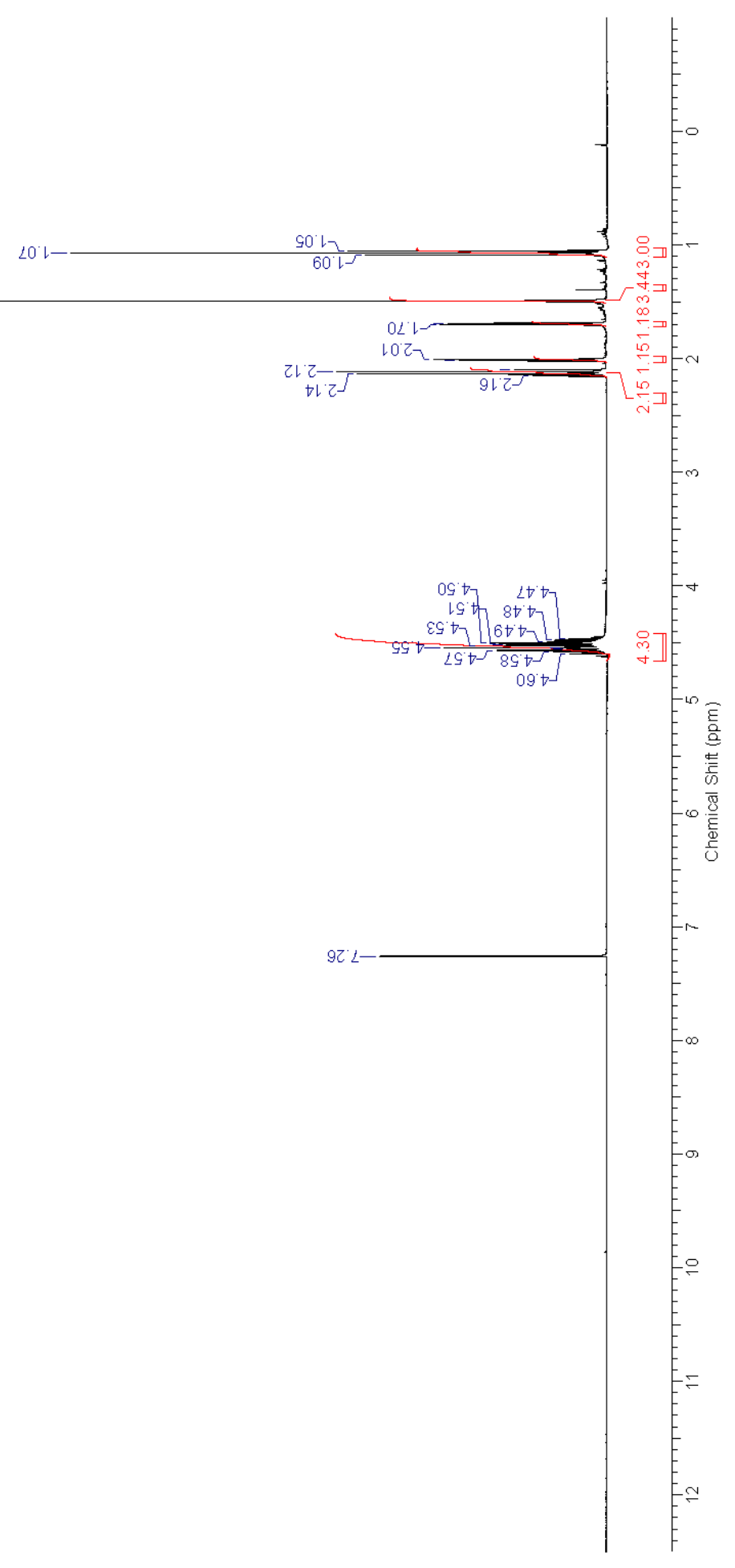



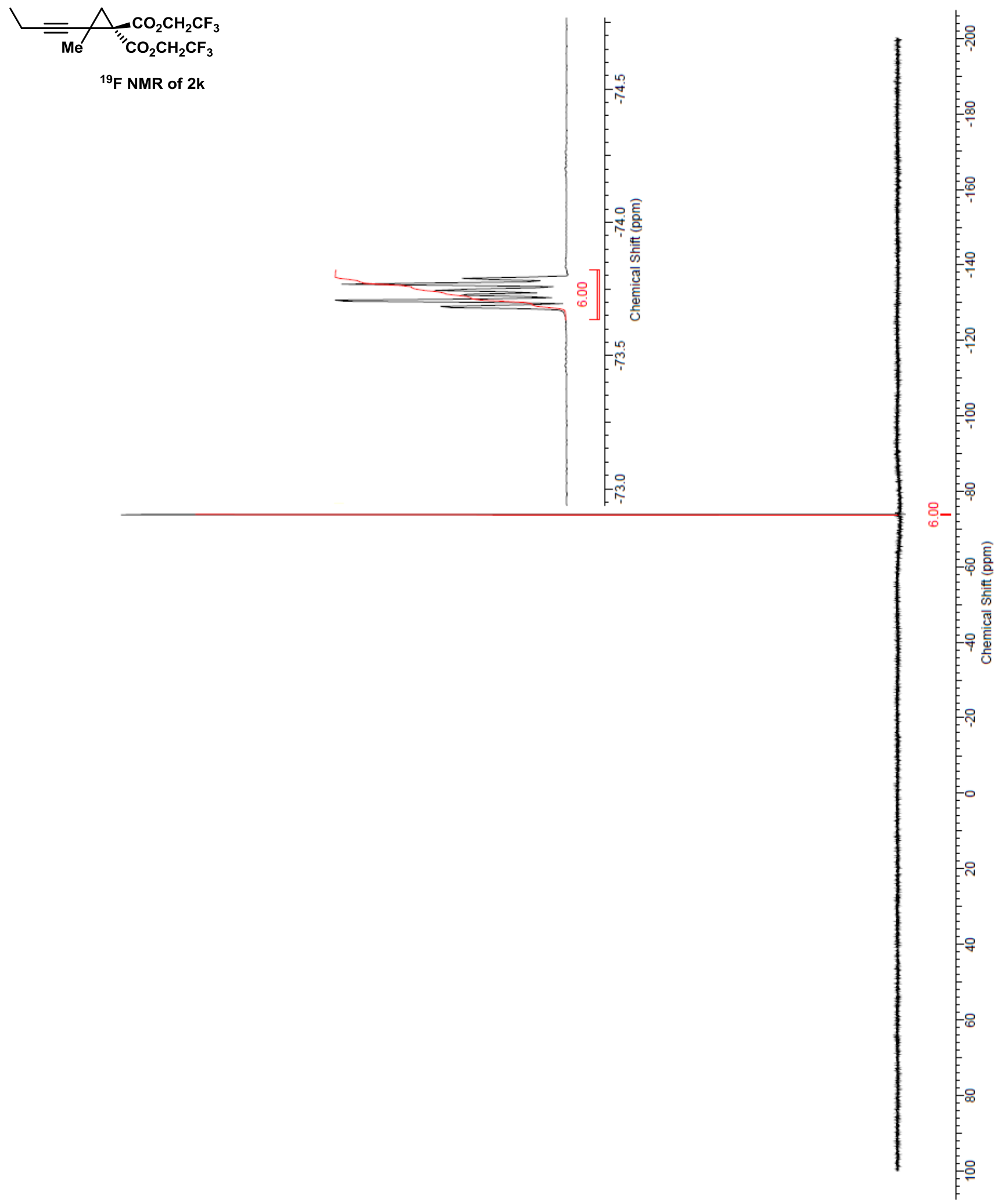
$=\underset{M e}{=} \triangle^{\prime \prime} \mathrm{CO}_{2} \mathrm{CH}_{2} \mathrm{CF}_{3}$
${ }^{13} \mathrm{C}$ NMR of $2 \mathrm{k}$

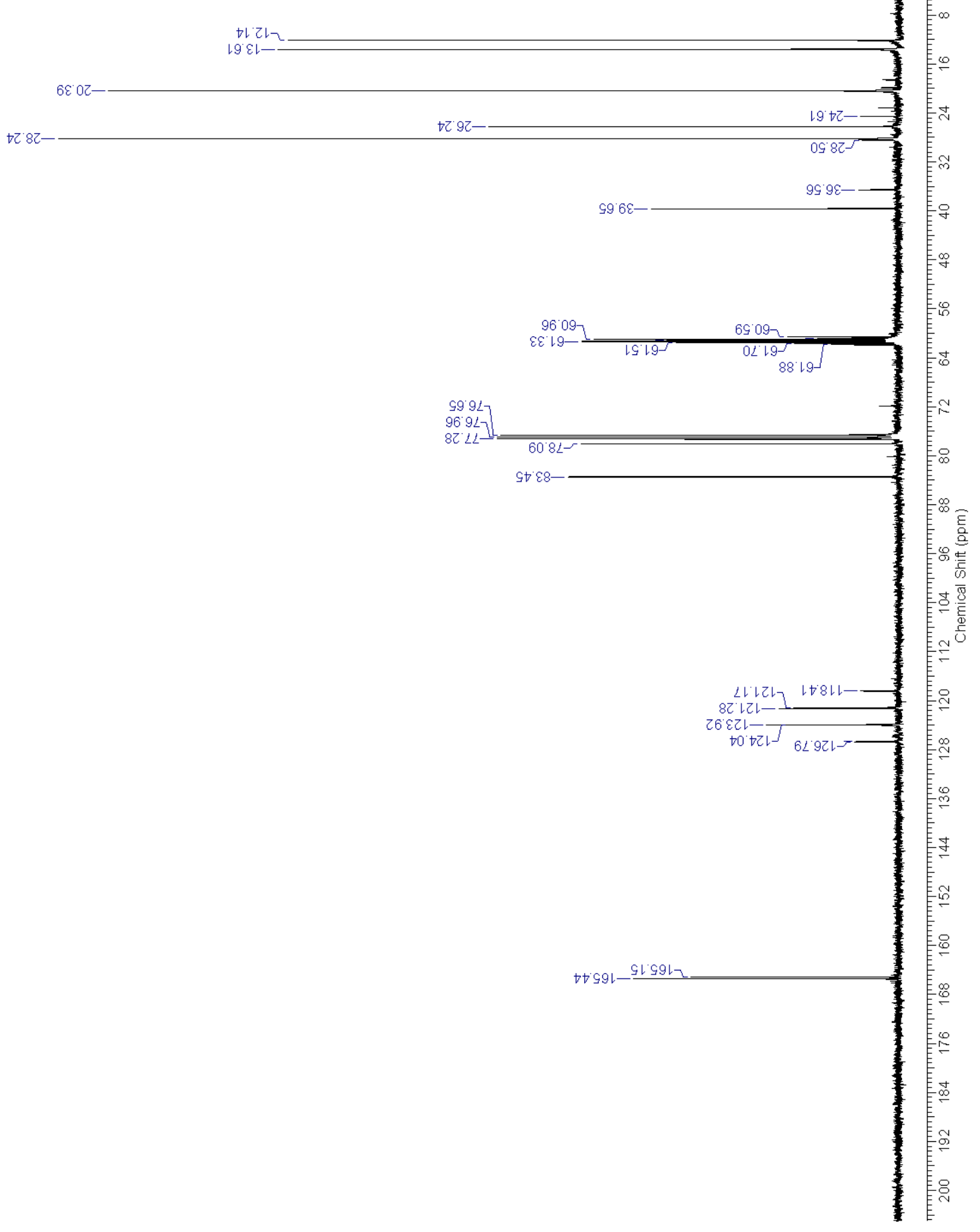




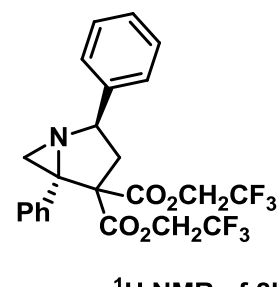

${ }^{1} \mathbf{H}$ NMR of $8 b$

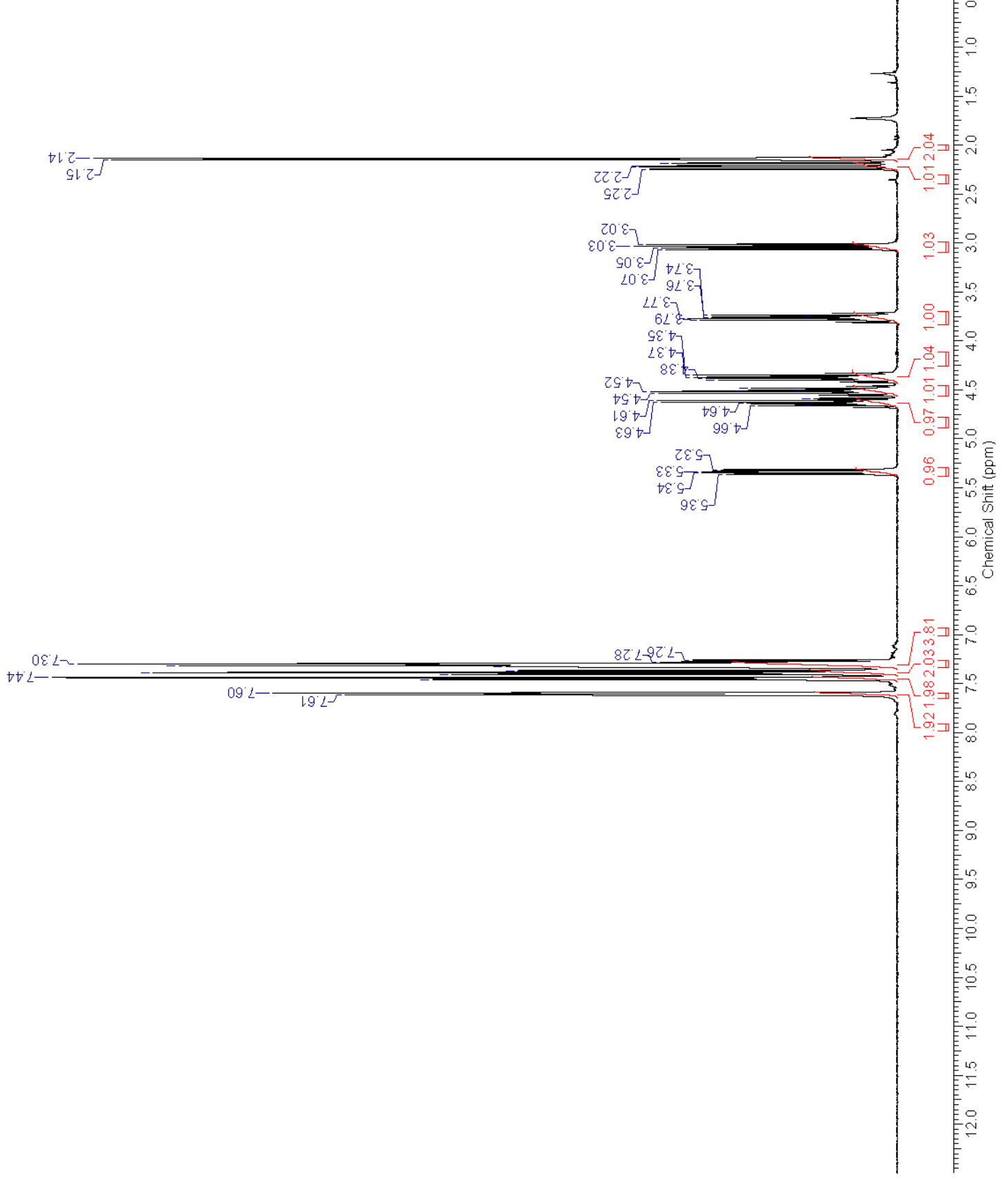




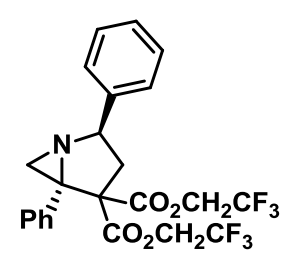

${ }^{19} \mathrm{~F}$ NMR of $8 b$
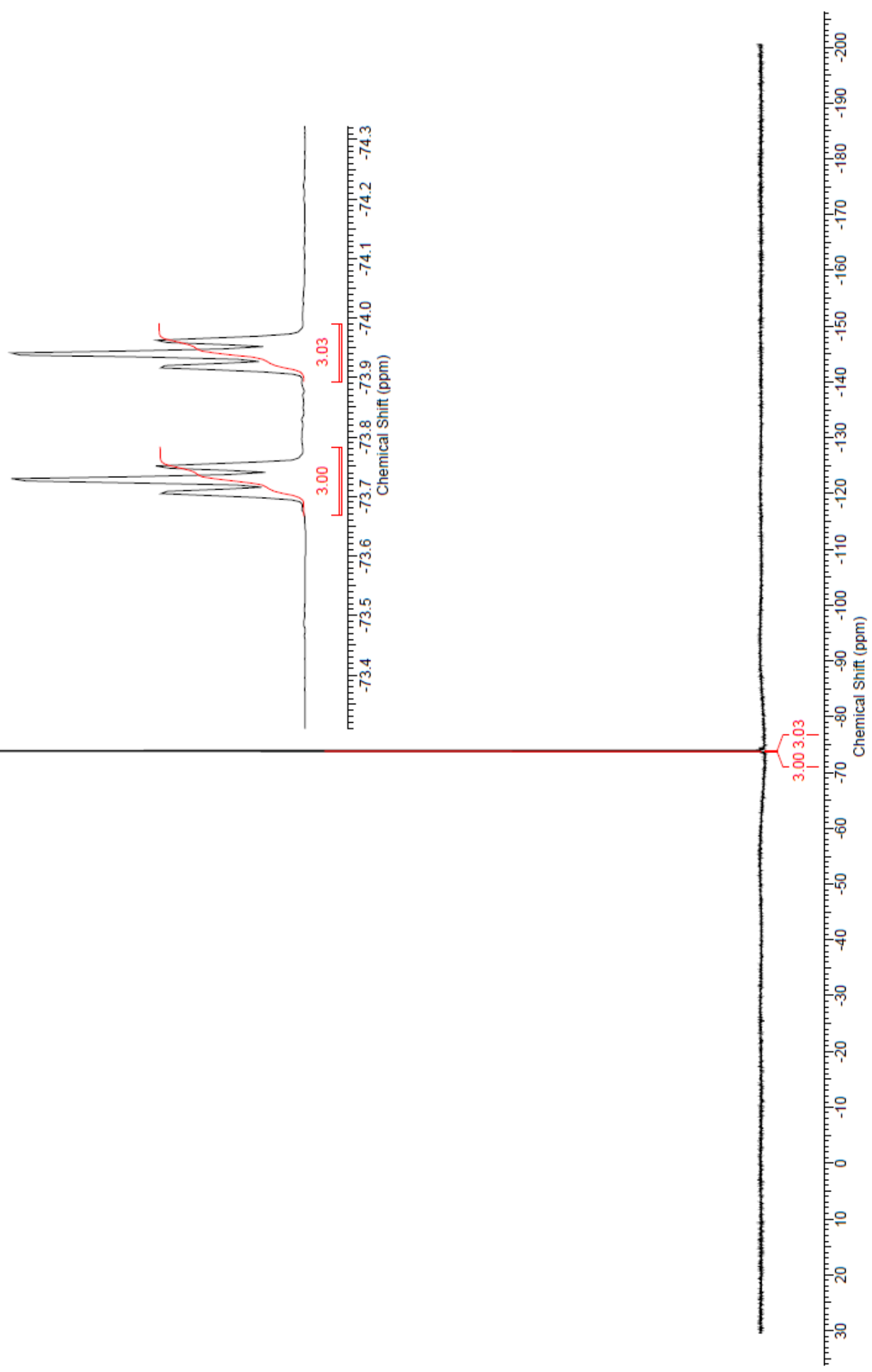

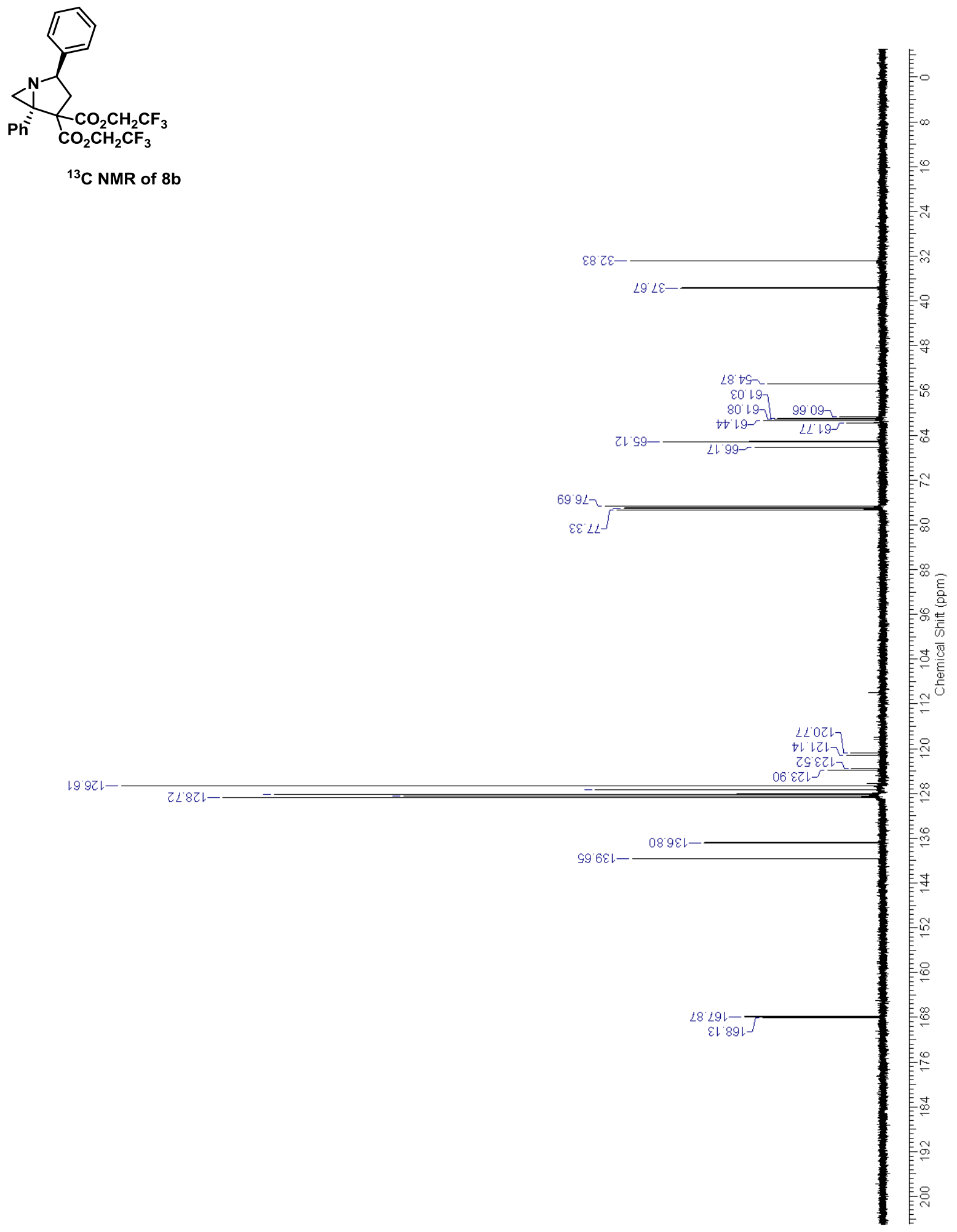


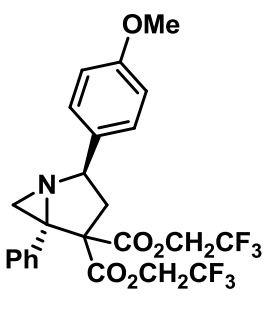

${ }^{1} \mathrm{H}$ NMR of $8 \mathrm{c}$

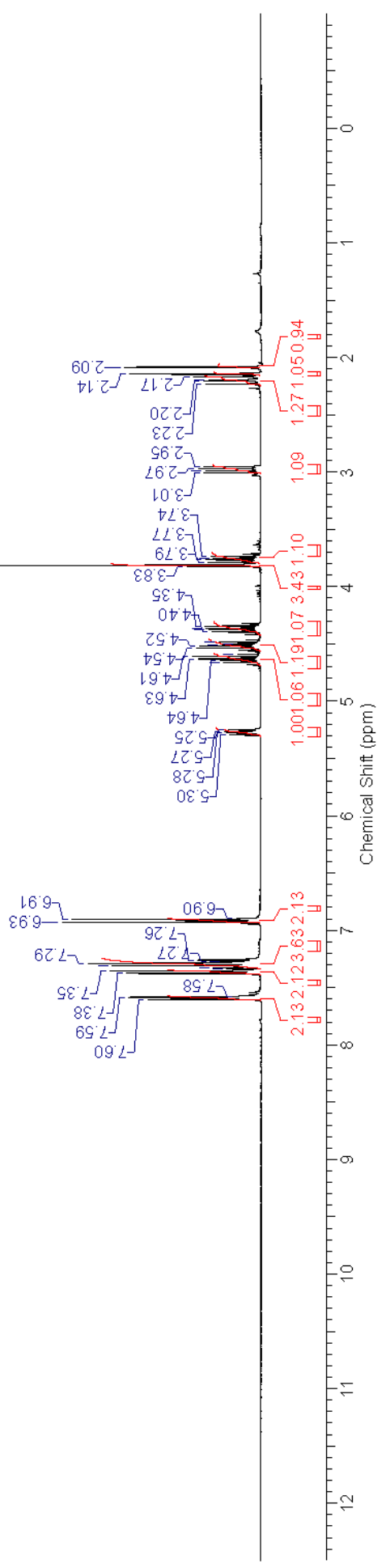




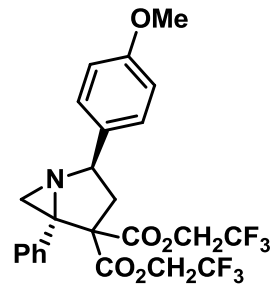

${ }^{19} \mathrm{~F}$ NMR of $8 \mathrm{c}$
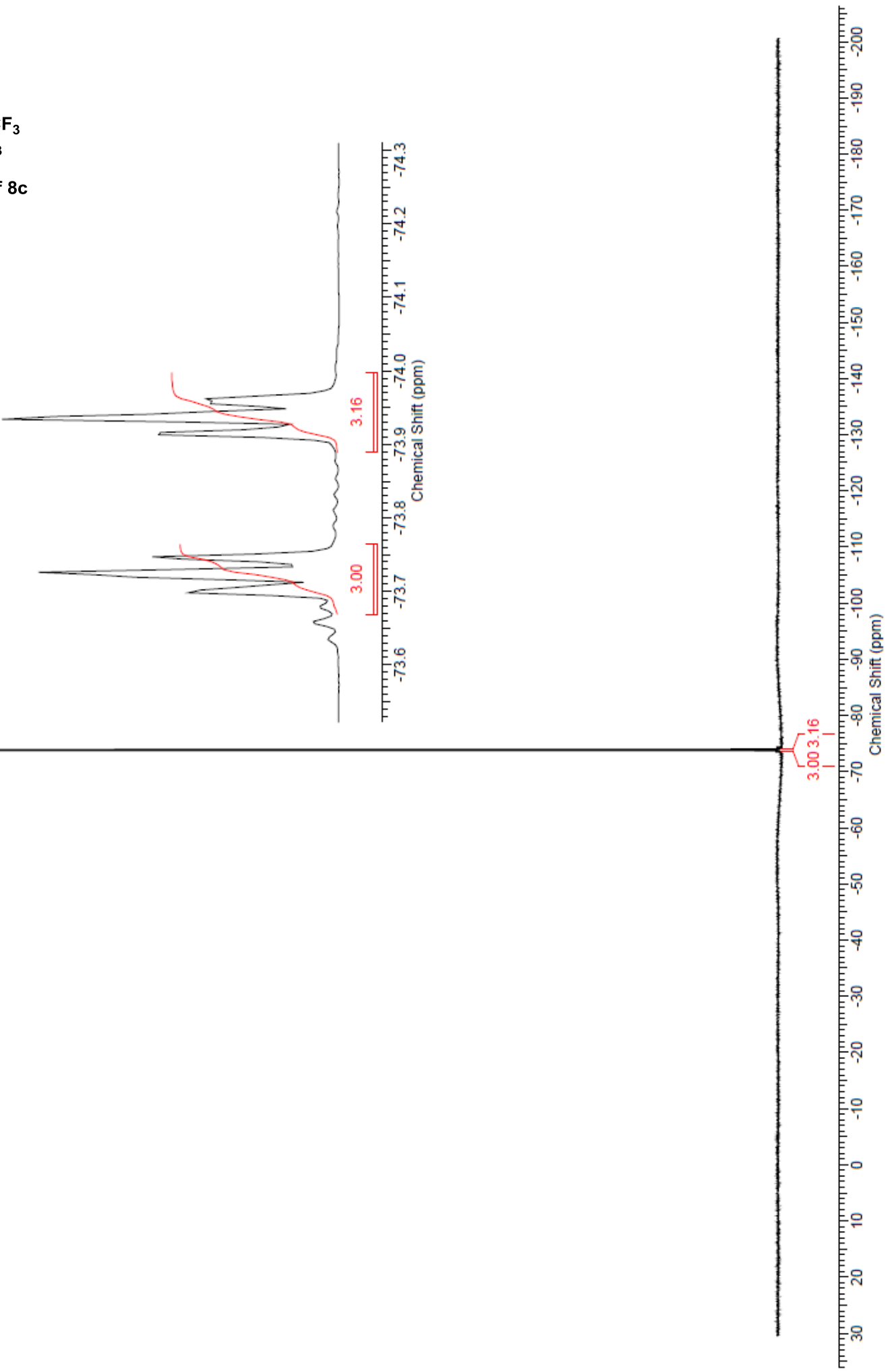


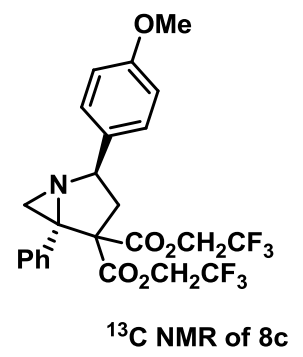

C NMR of $8 c$

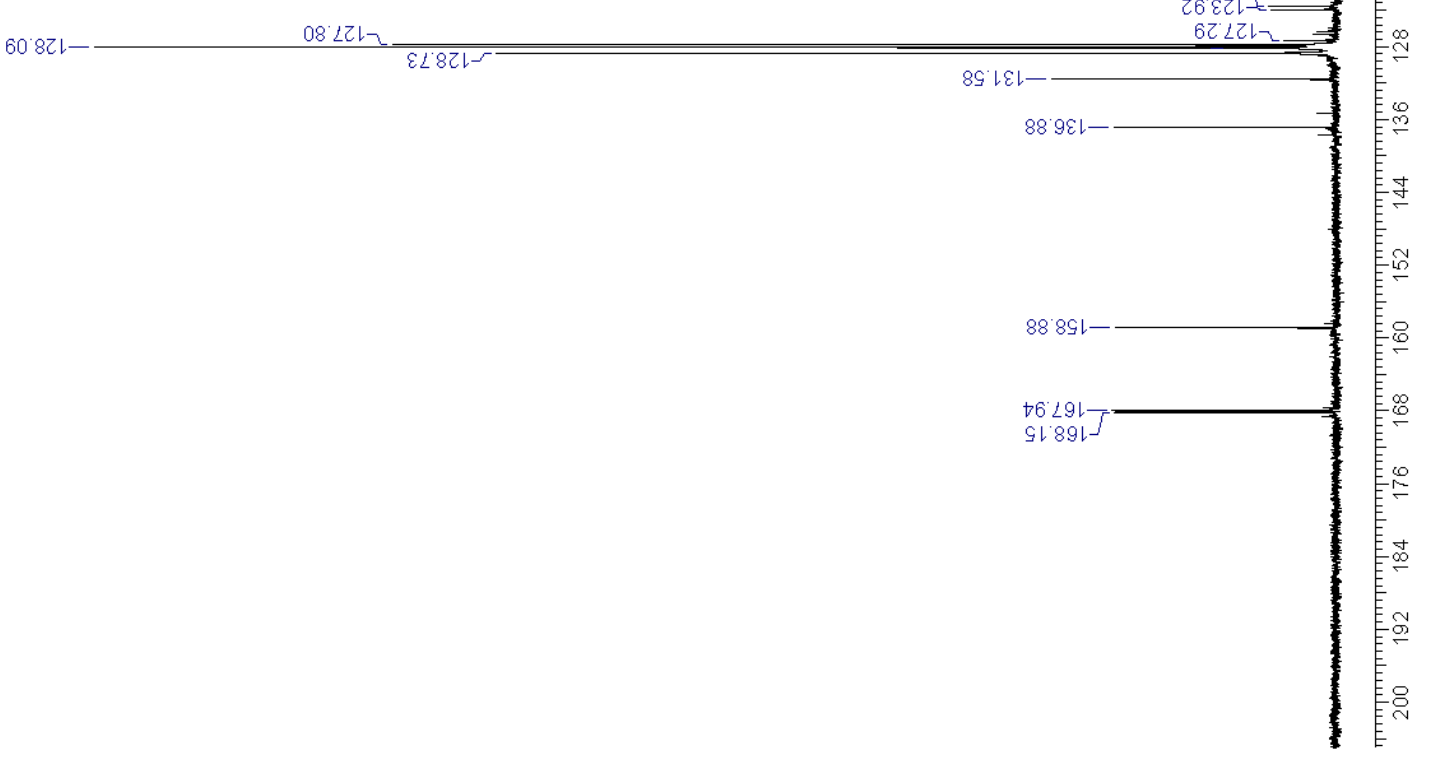




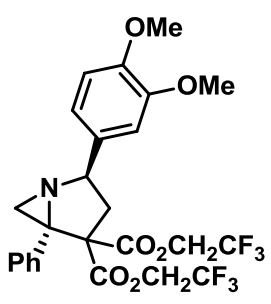

${ }^{1} \mathrm{H}$ NMR of $\mathbf{8 d}$

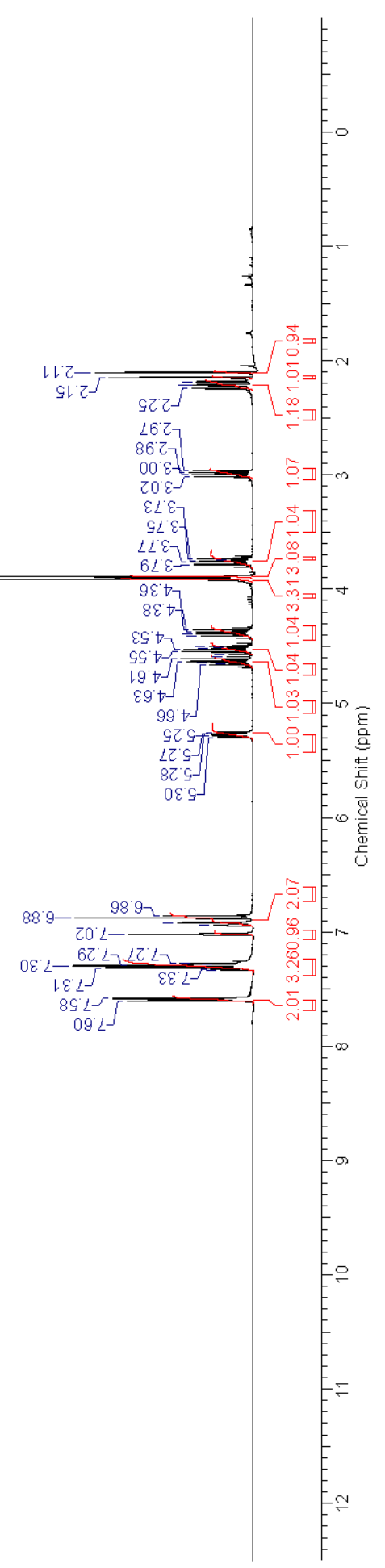



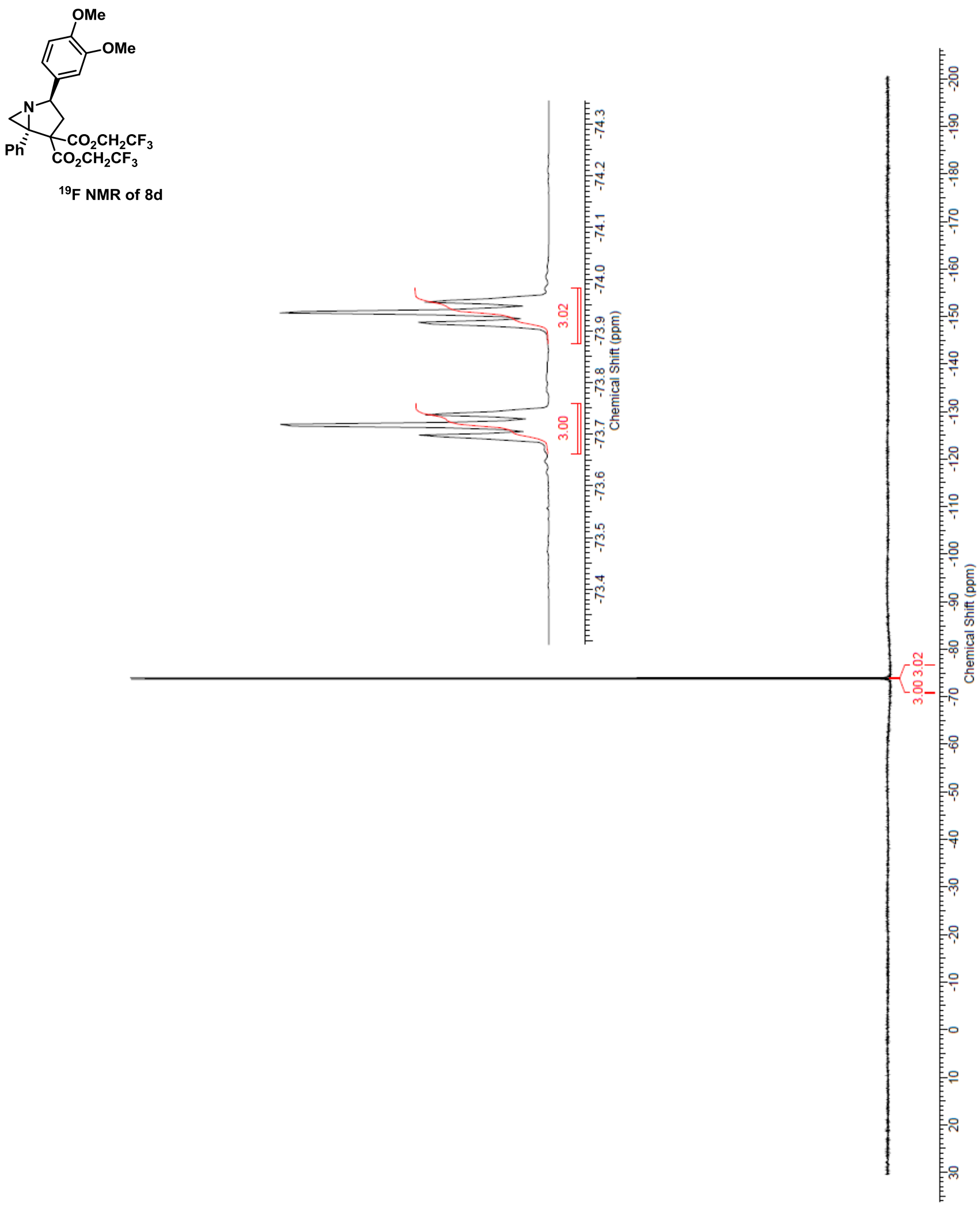


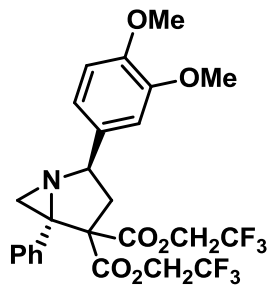

${ }^{13} \mathrm{C}$ NMR of $8 d$

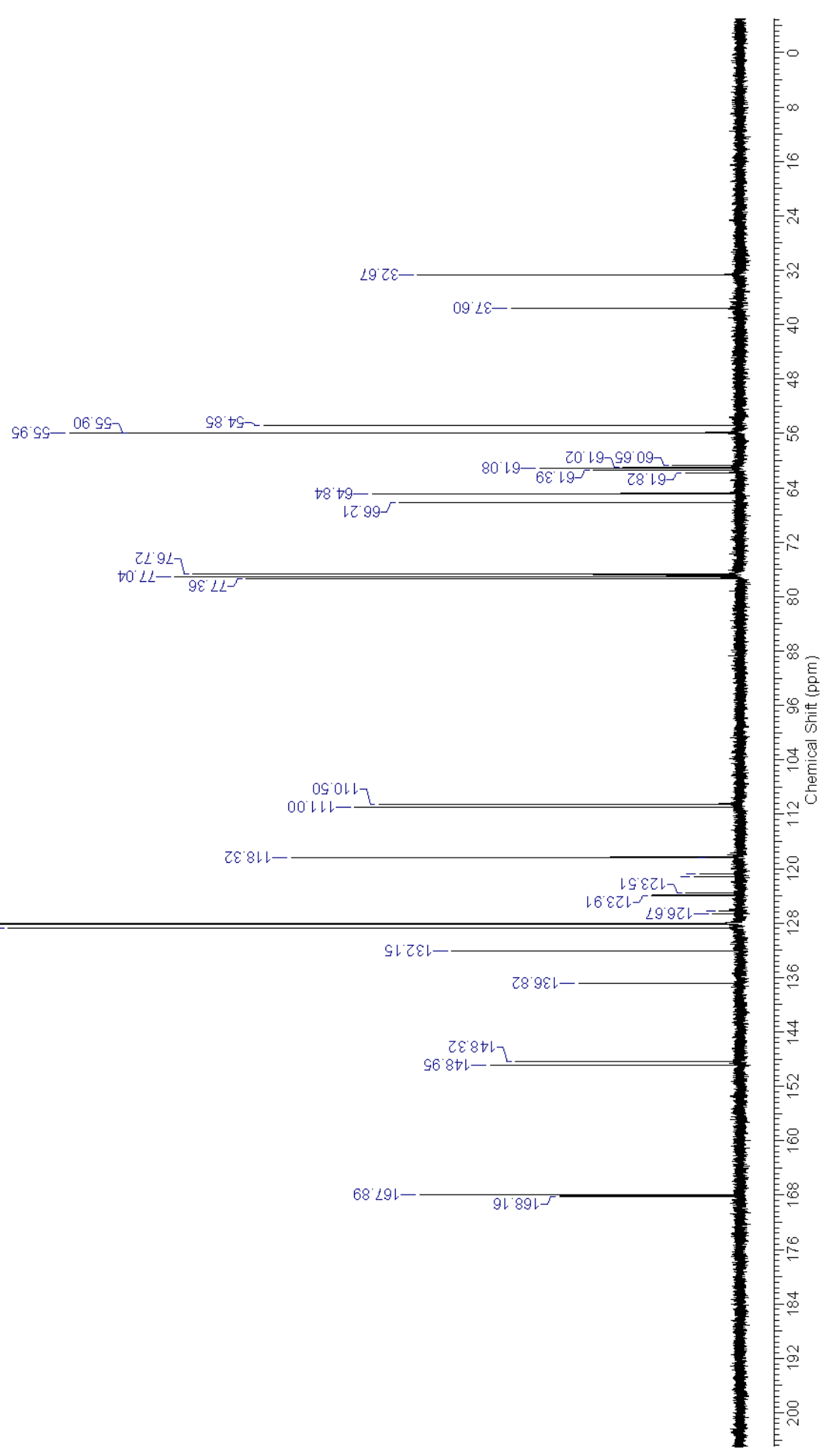



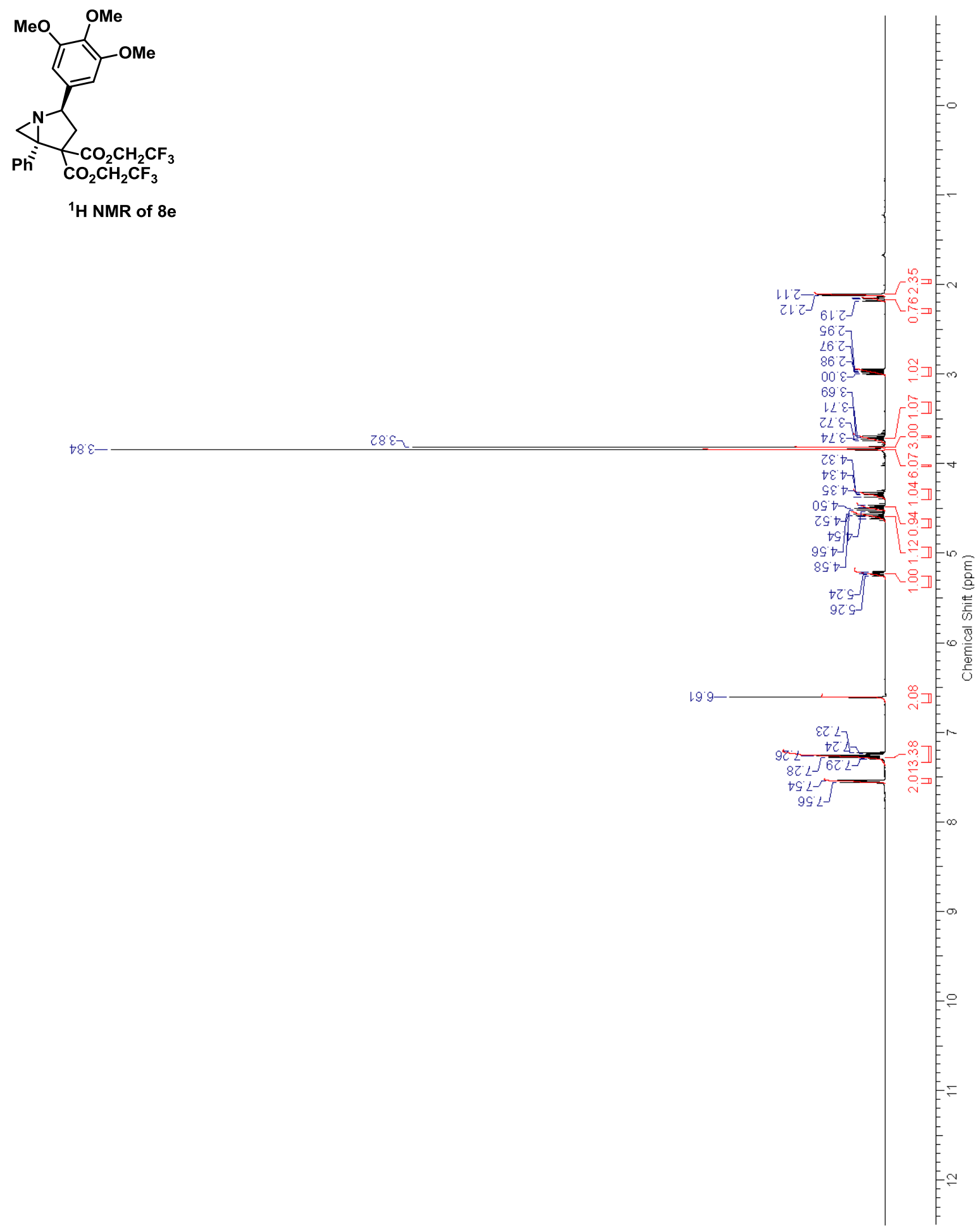

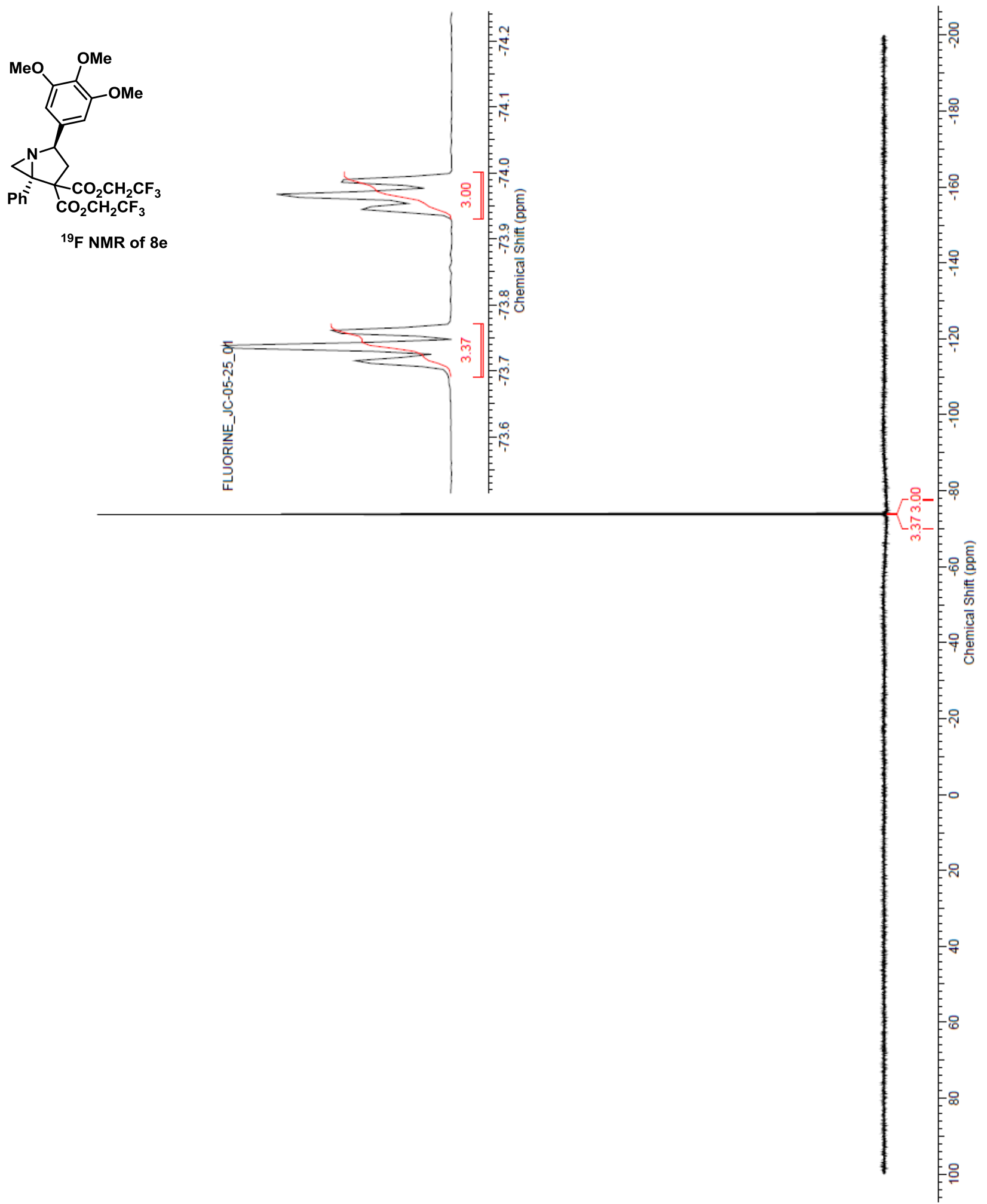


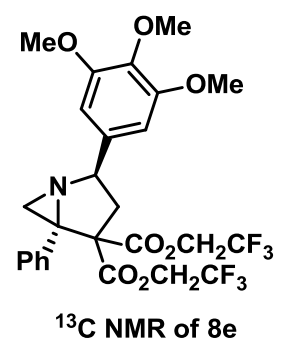

61.99

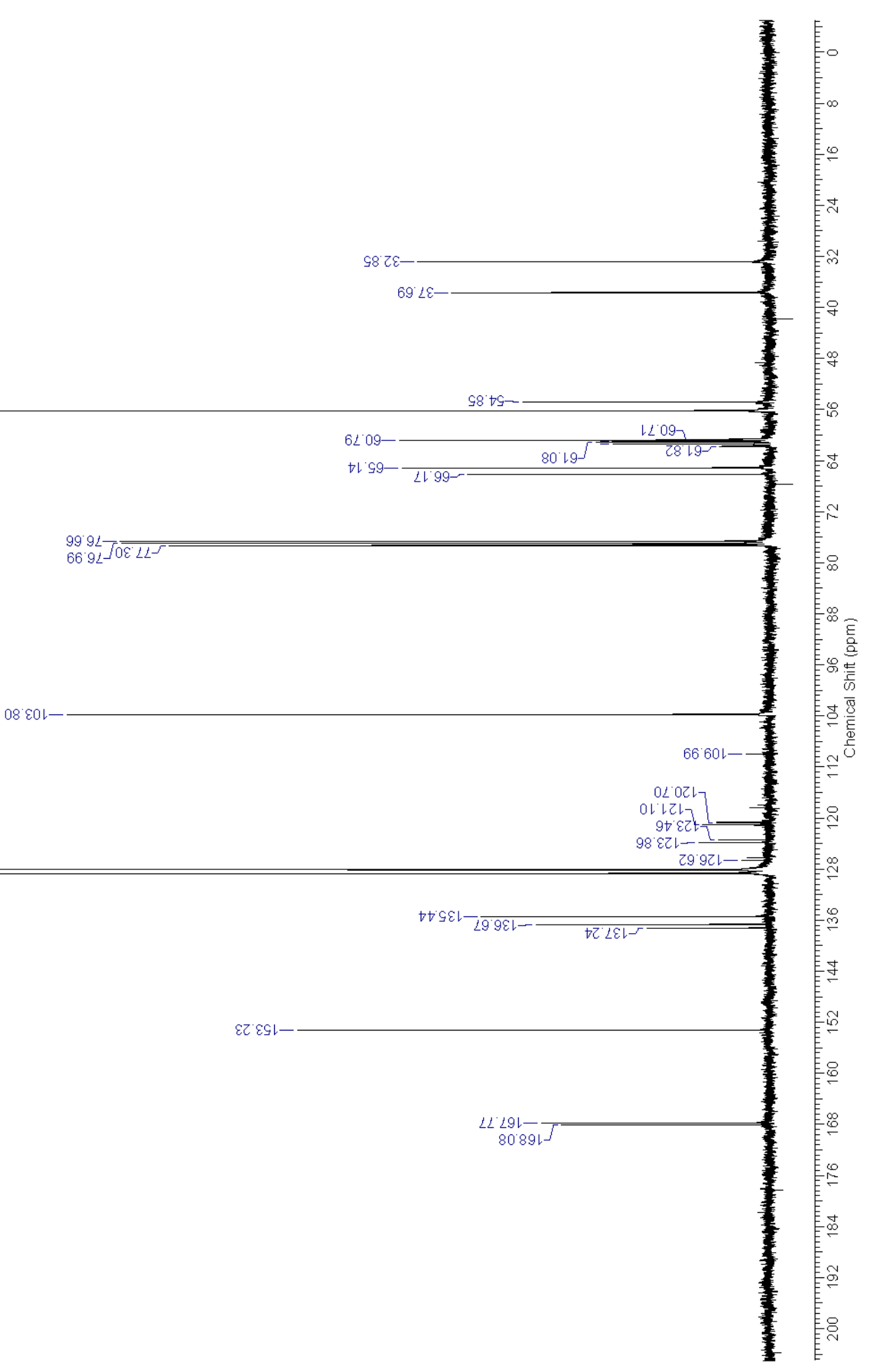




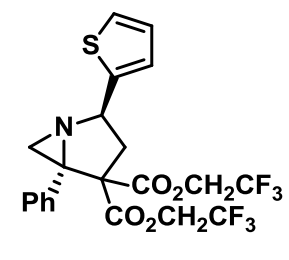

${ }^{1} \mathrm{H}$ NMR of $8 f$

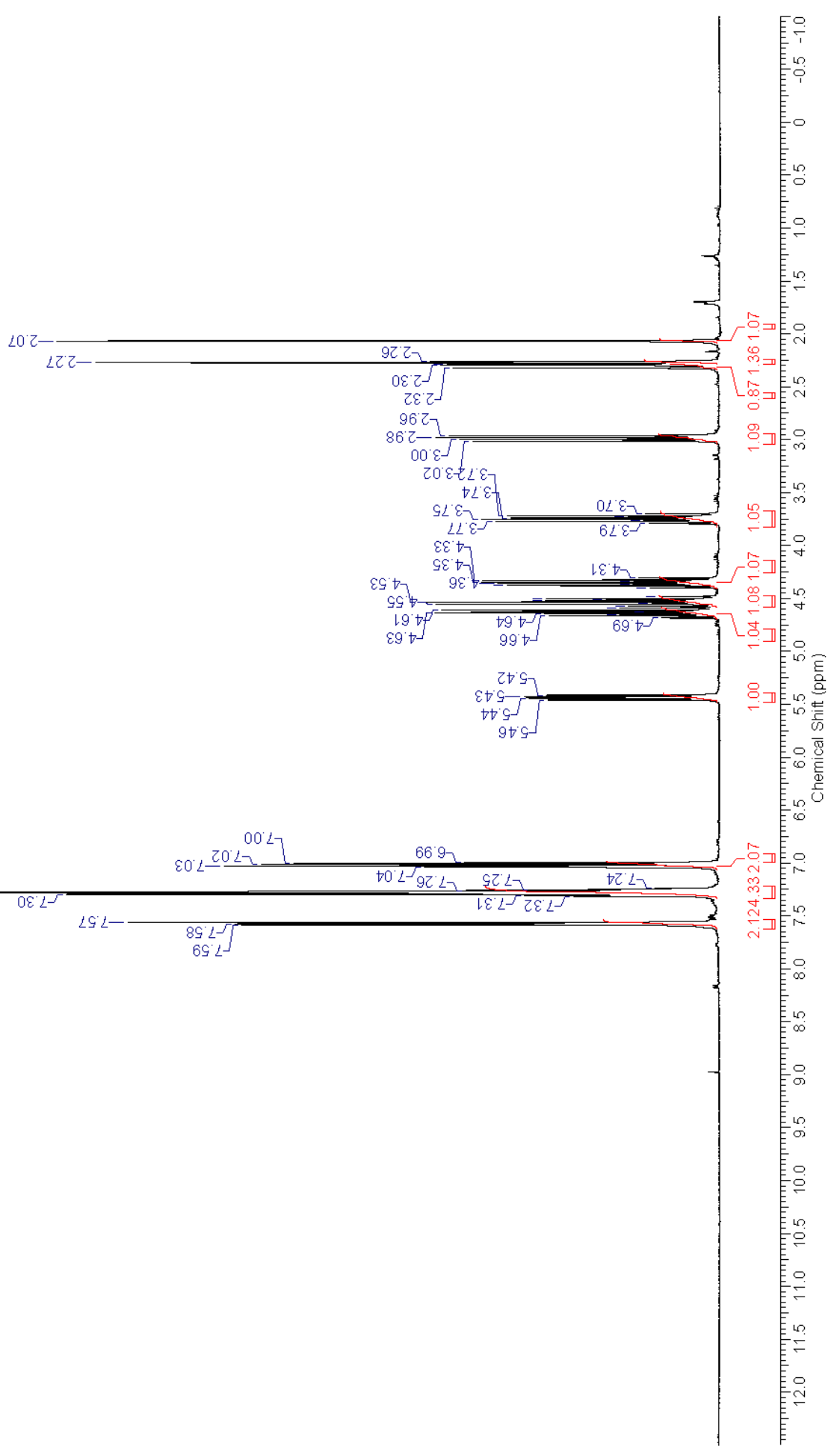



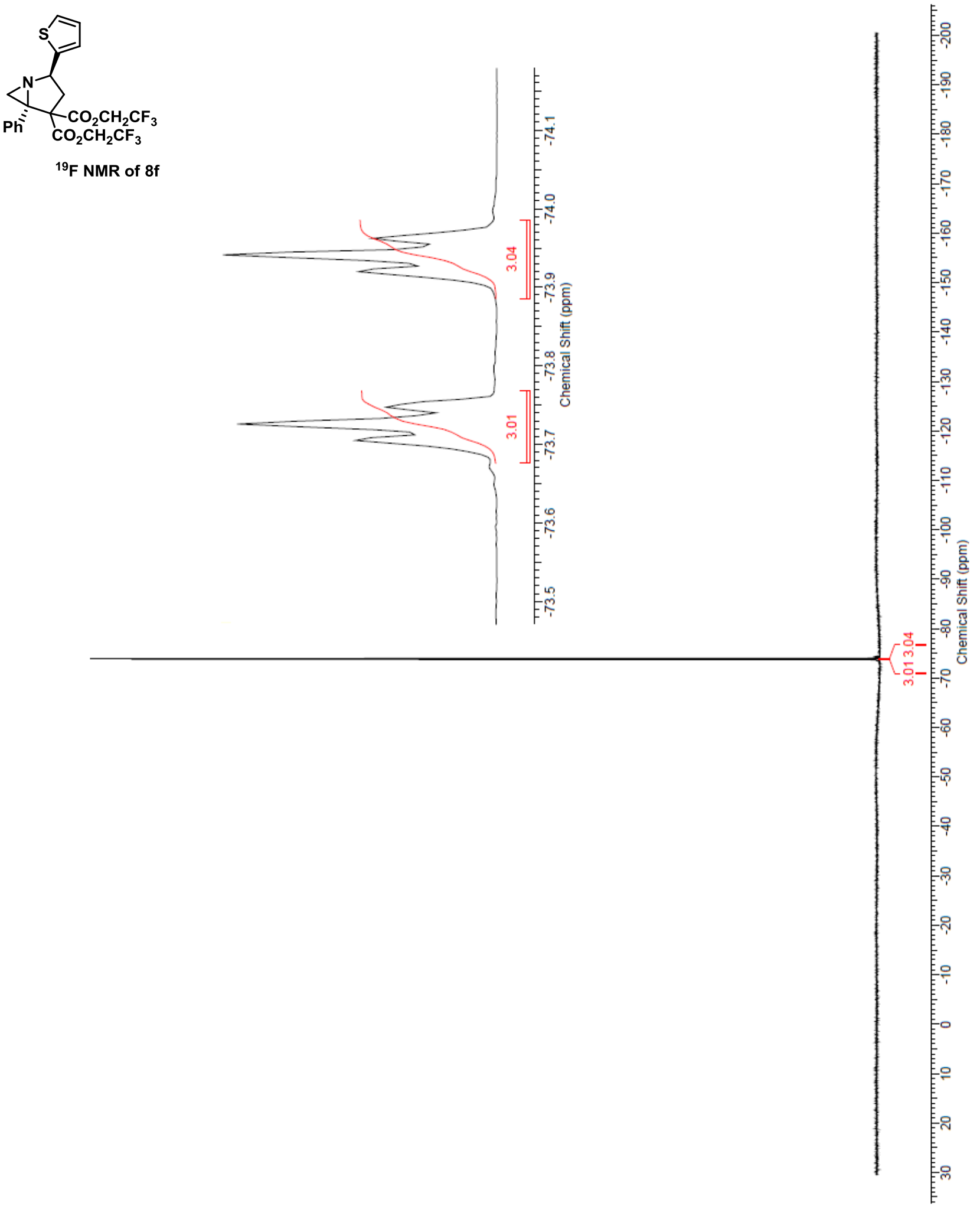


$$
\text { 和 }
$$



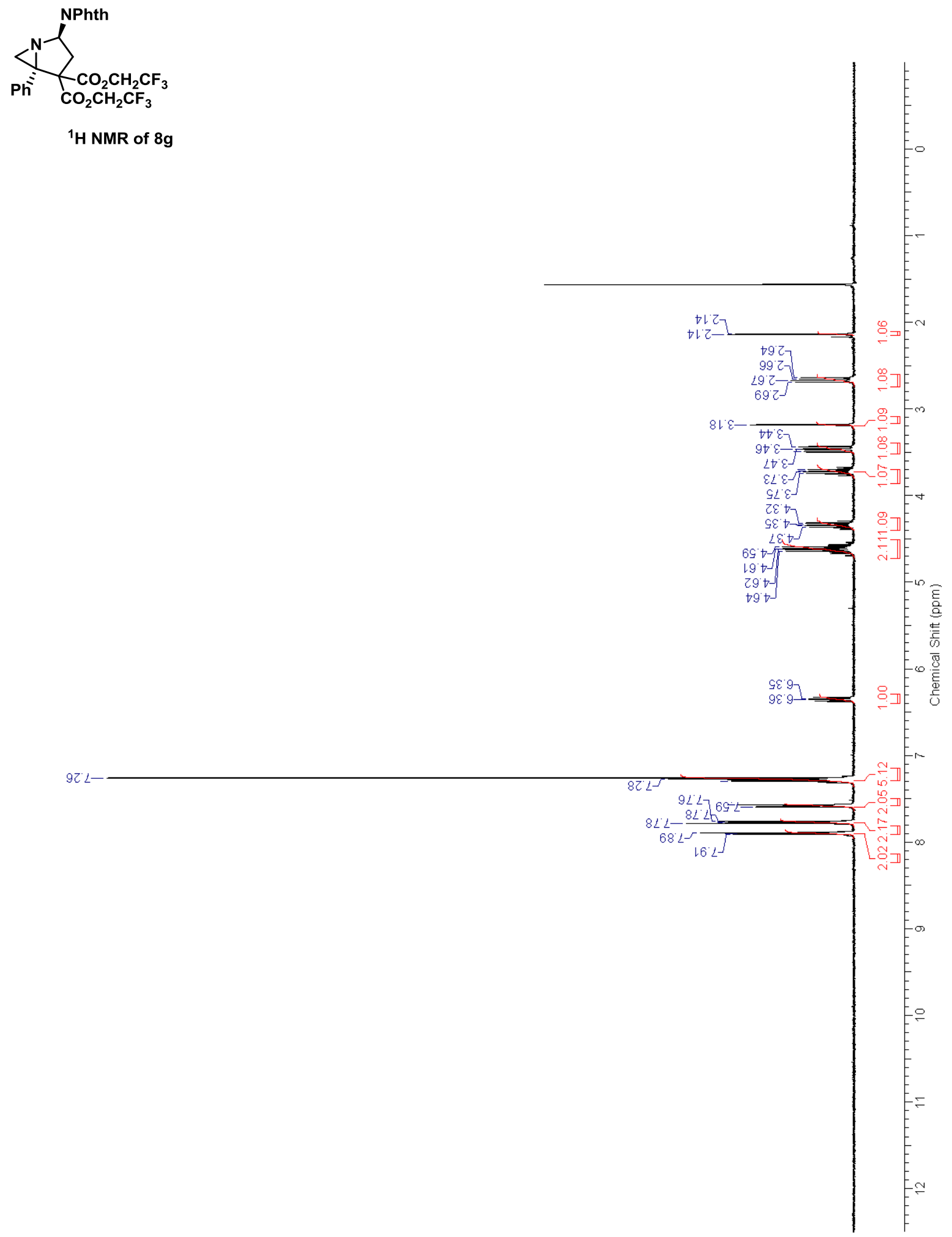


$$
=11
$$




$$
1
$$


רo

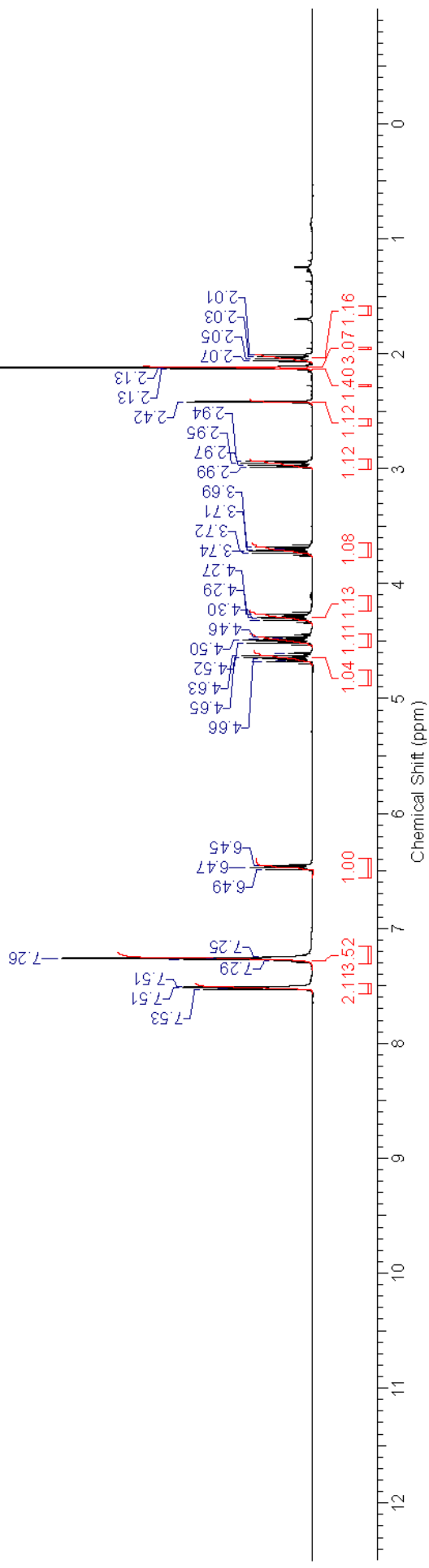



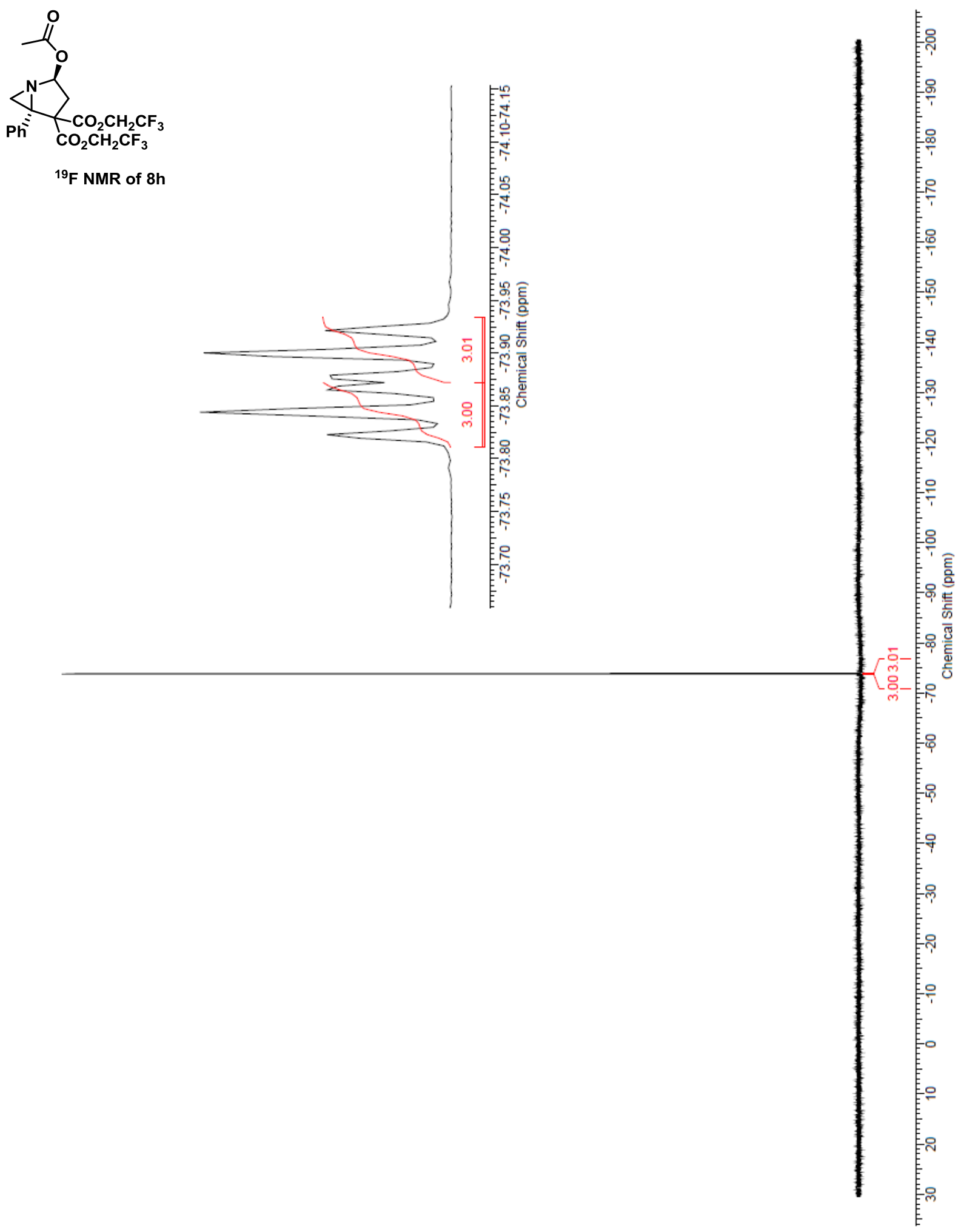

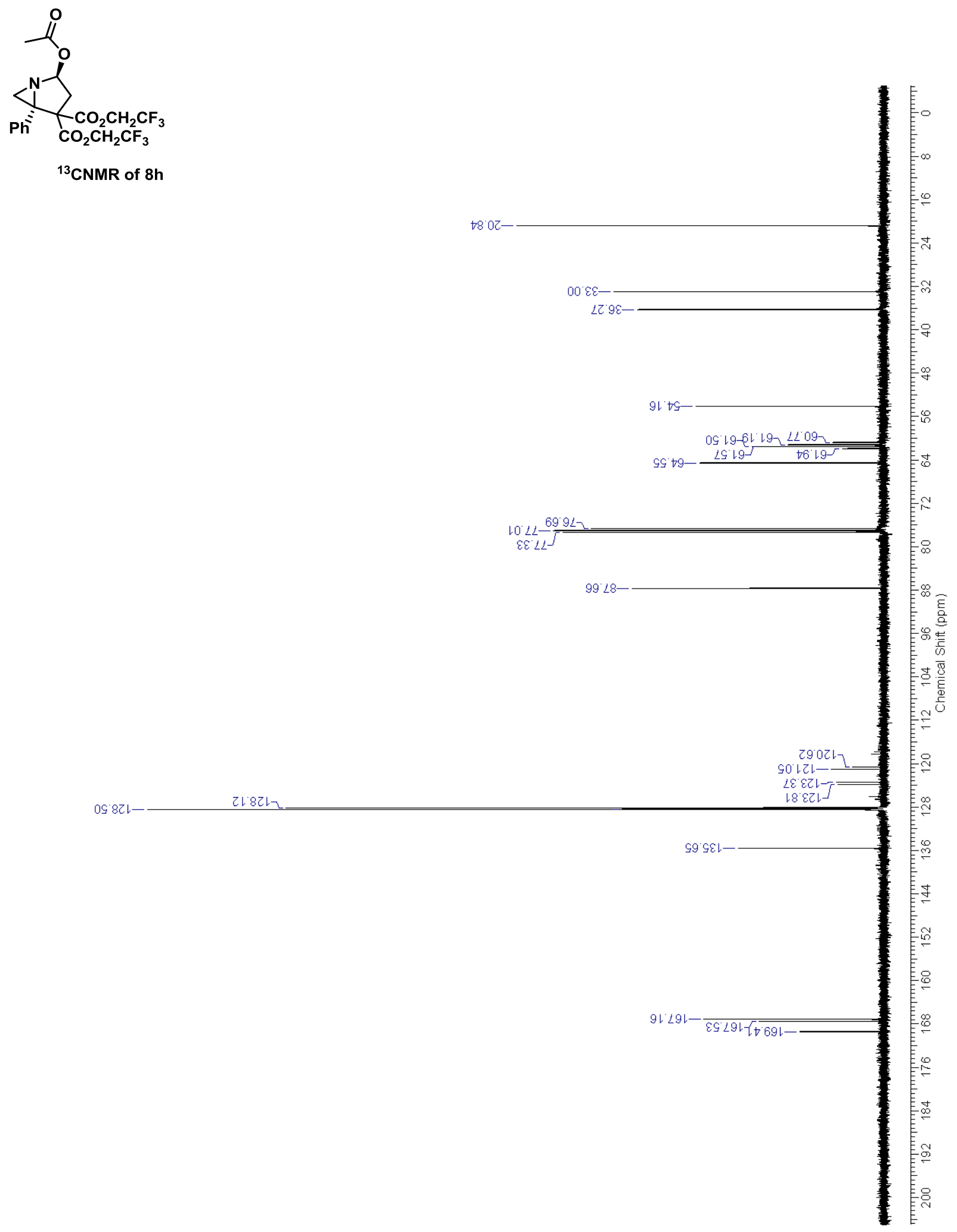

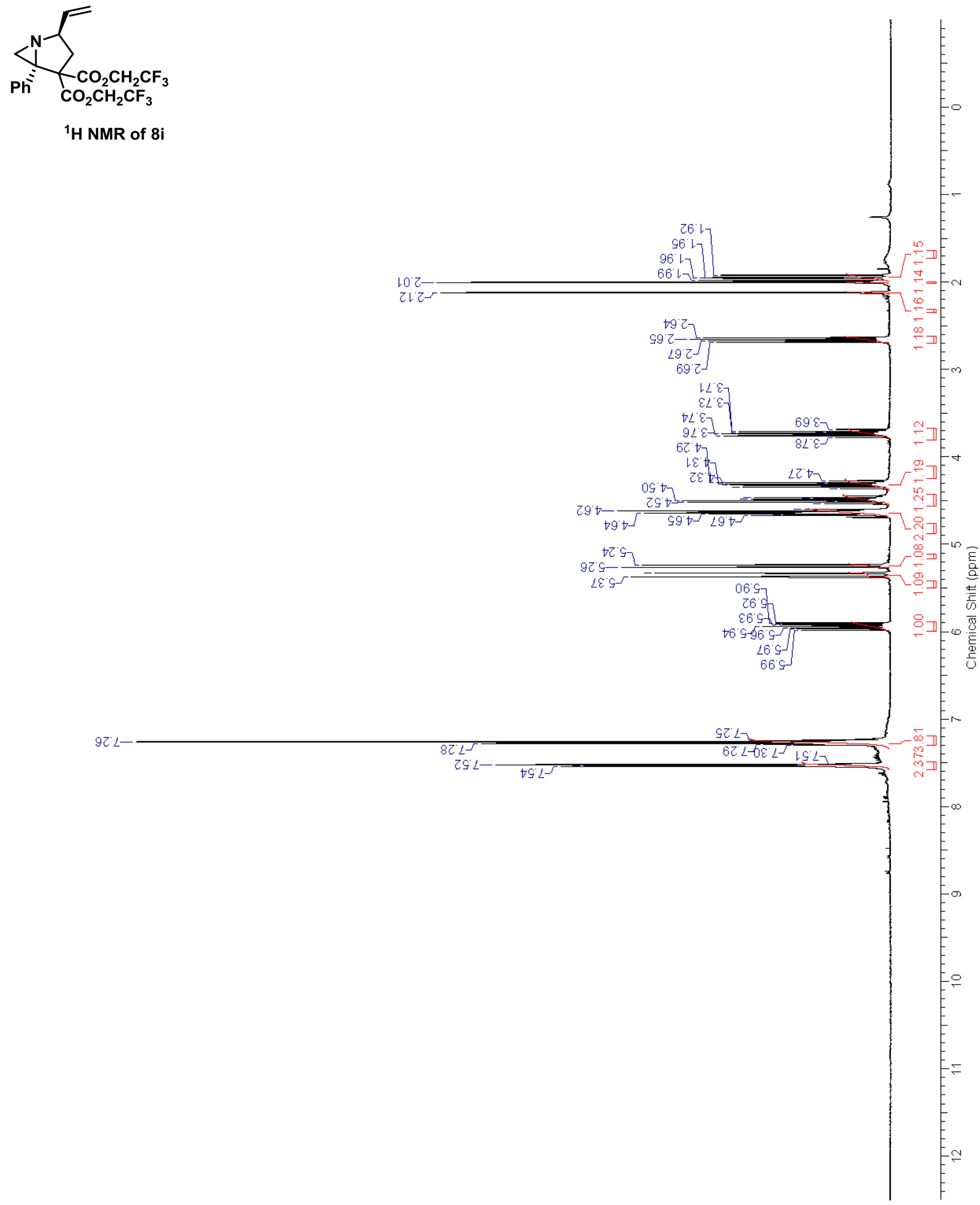


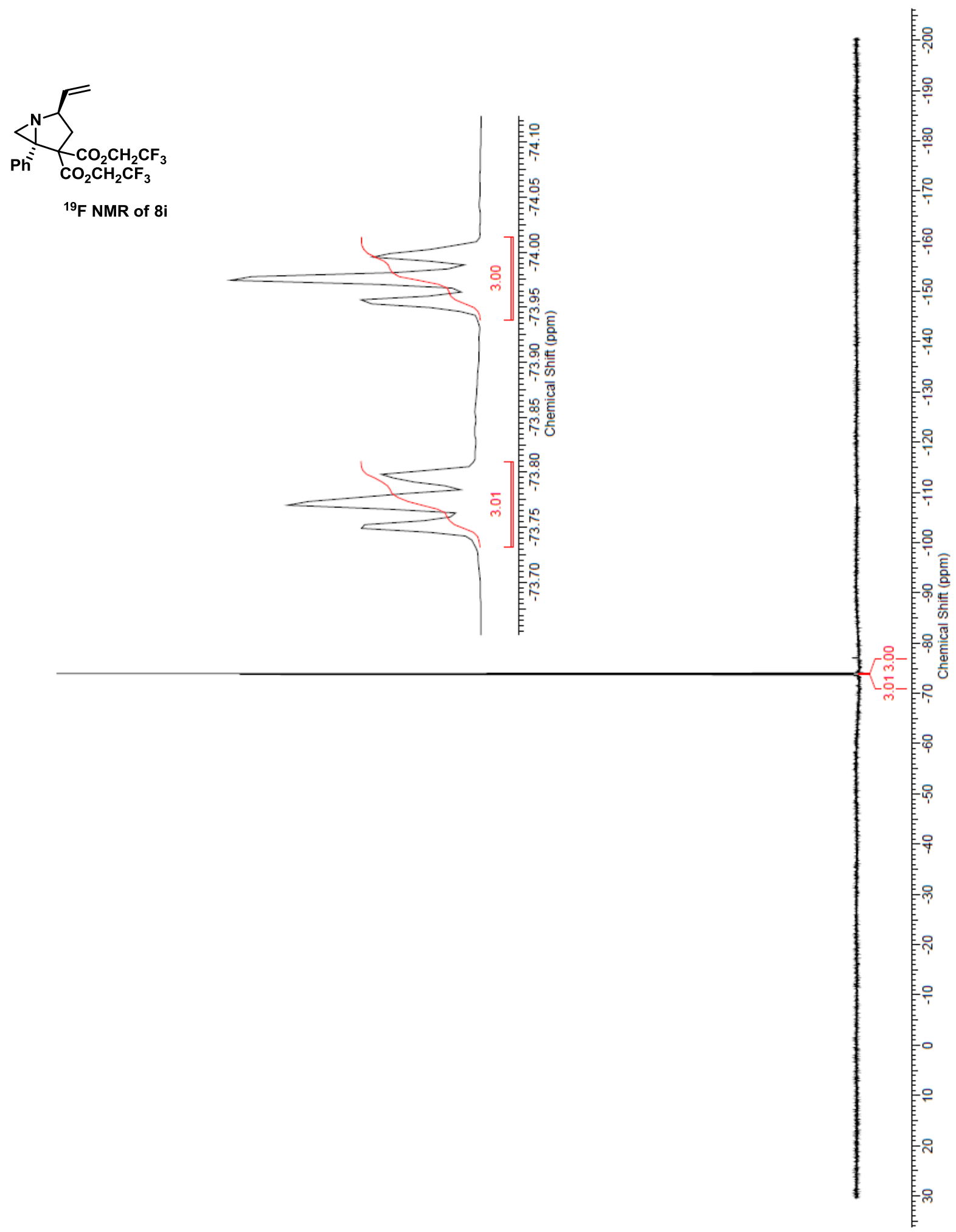


<smiles>C=CC1CC(C(=O)OCCC(F)(F)F)(C(=O)OCCC(F)(F)F)C2(c3ccccc3)CN12</smiles>

${ }^{13} \mathrm{C}$ NMR of $8 \mathrm{i}$

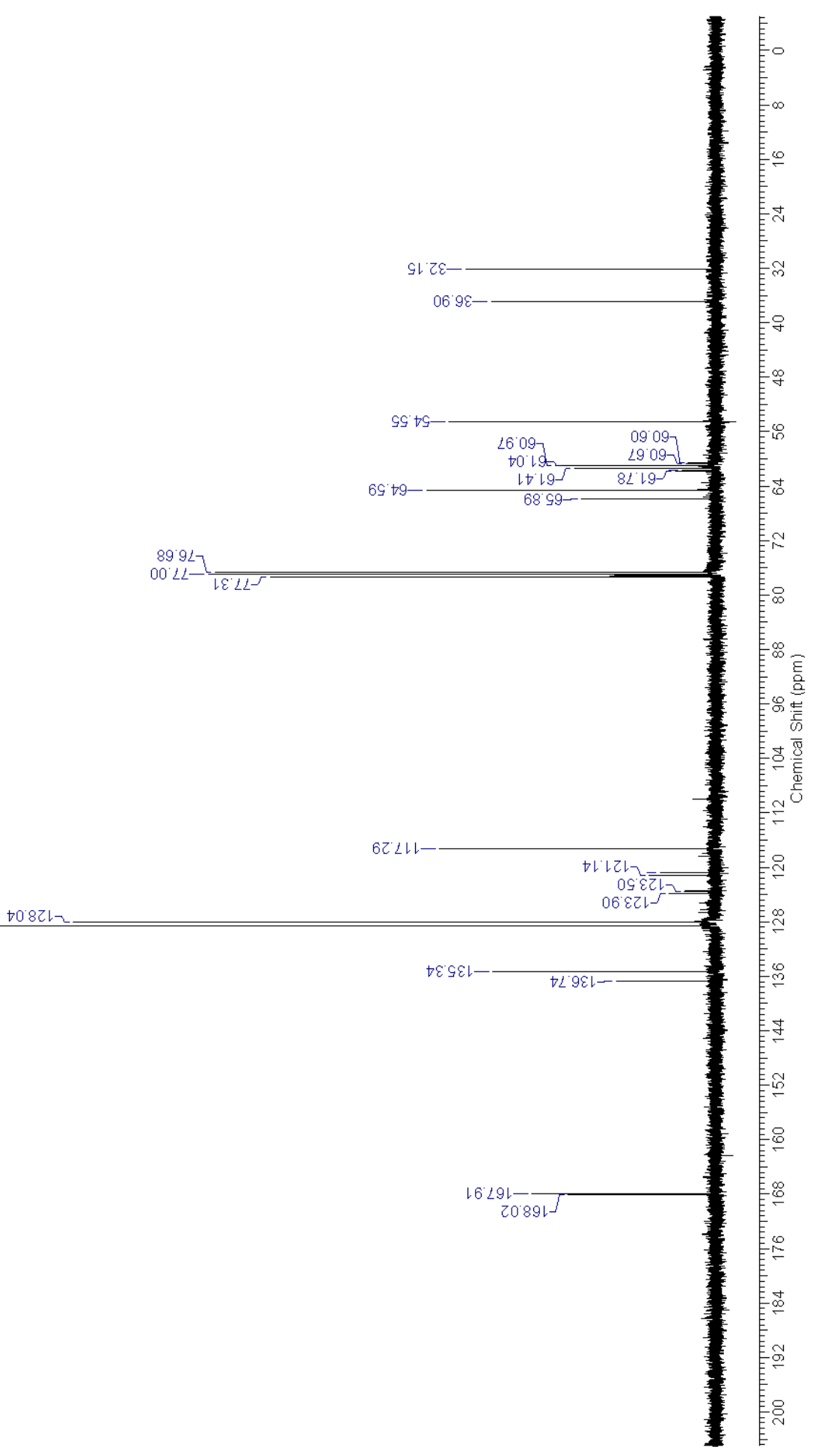

76 


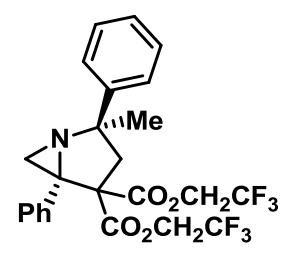

${ }^{1} \mathrm{H}$ NMR of $\mathbf{8 j}$

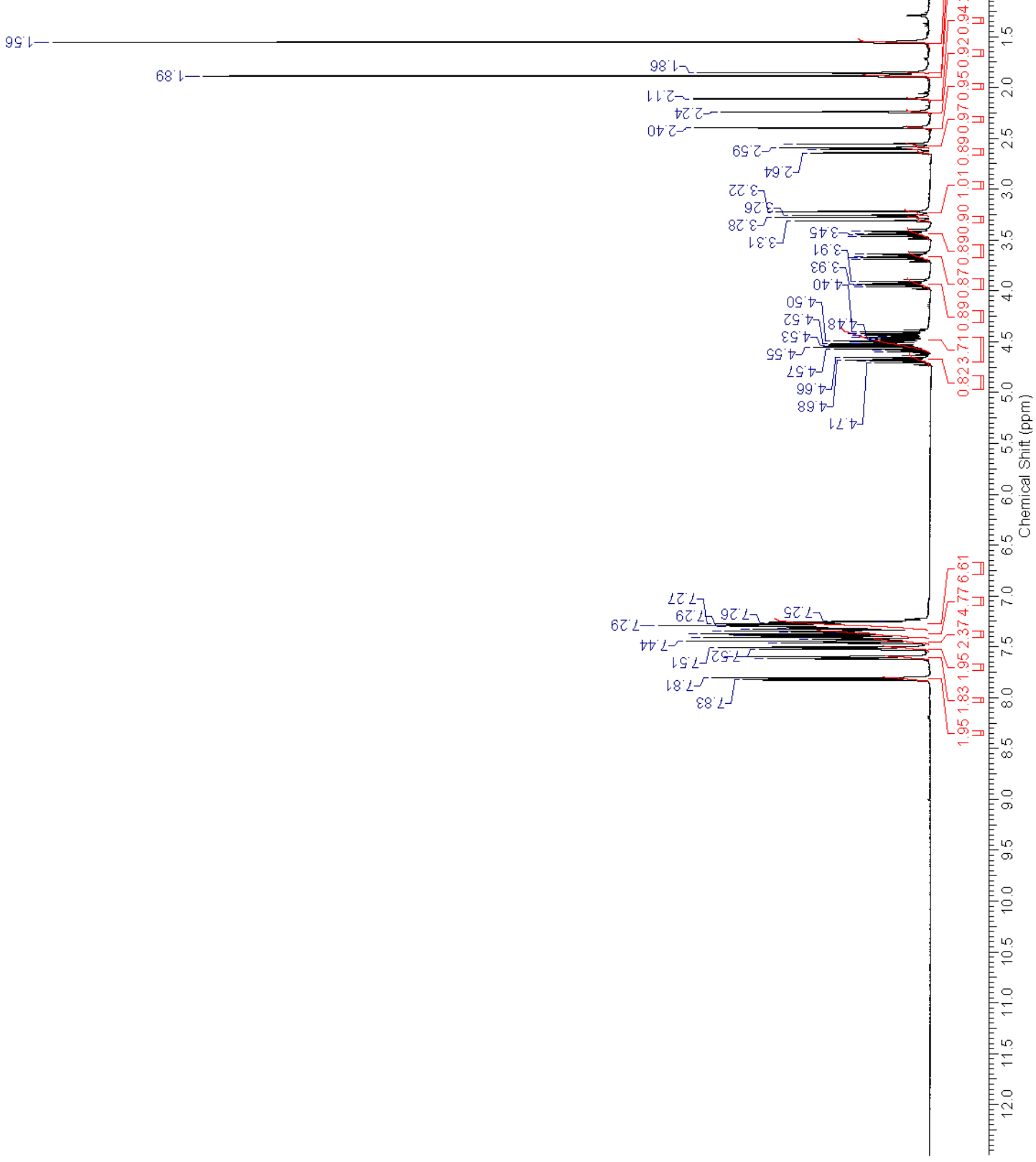



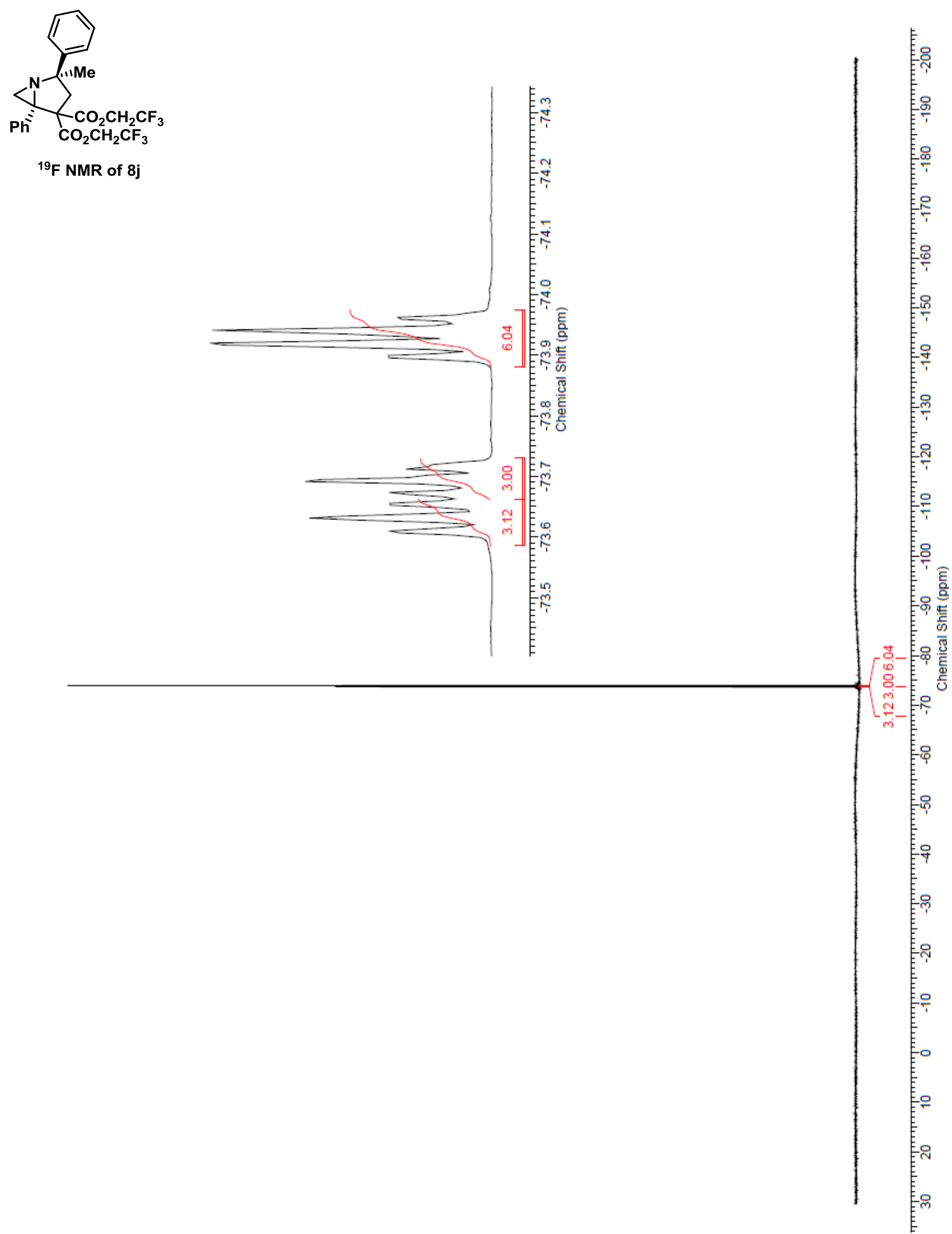


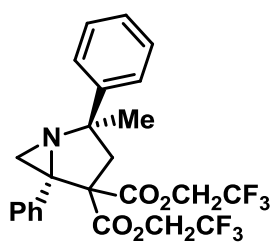

${ }^{13} \mathrm{C}$ NMR of $8 \mathrm{j}$

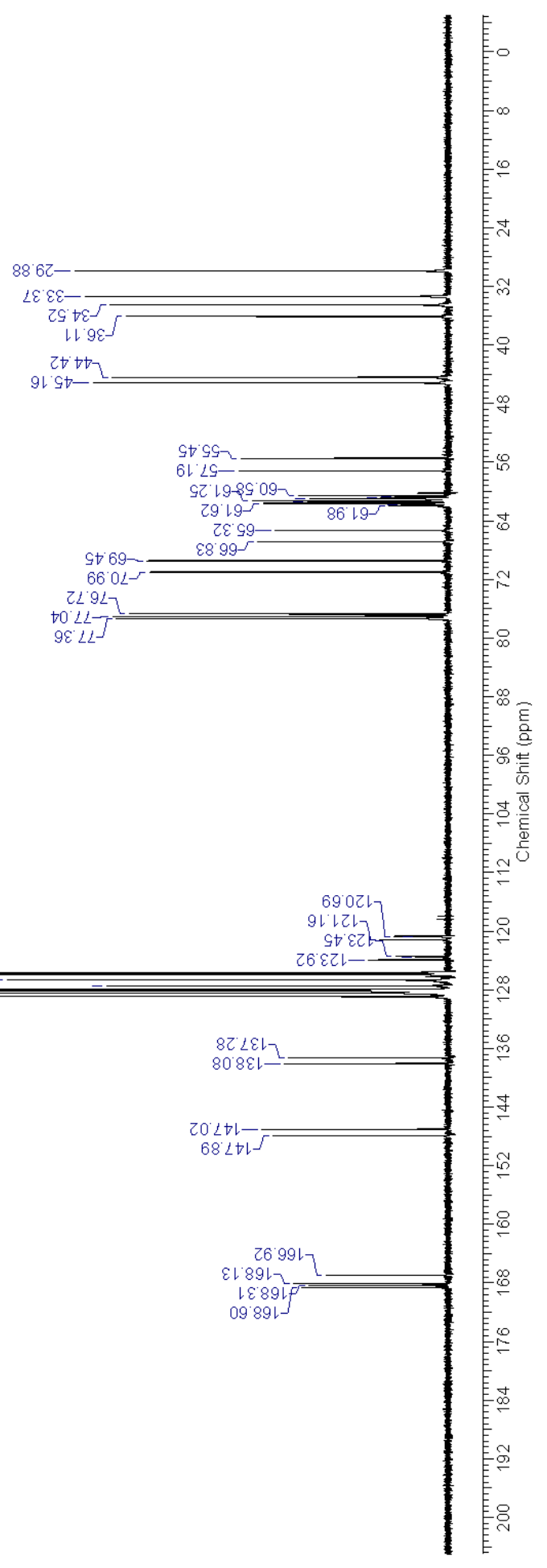



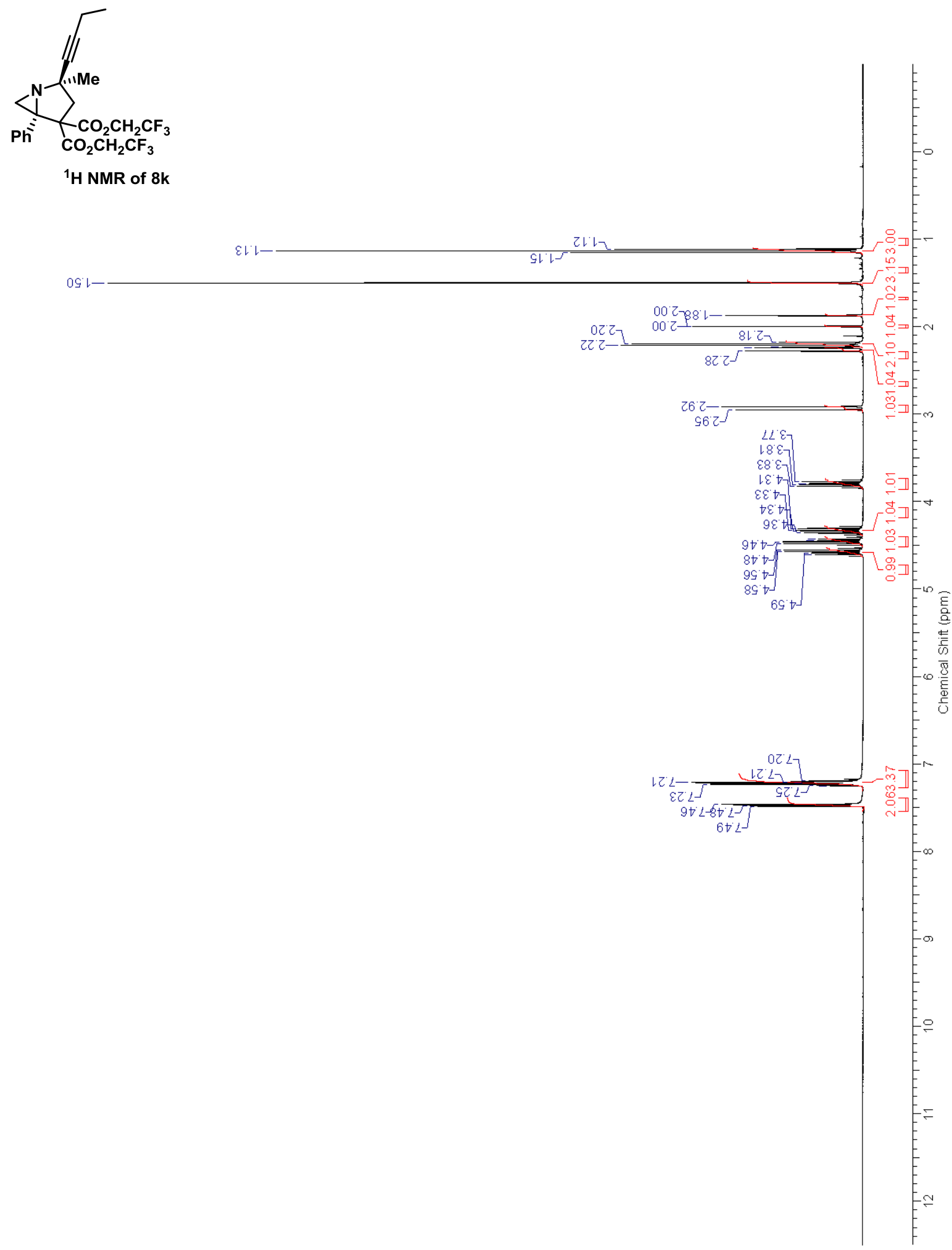

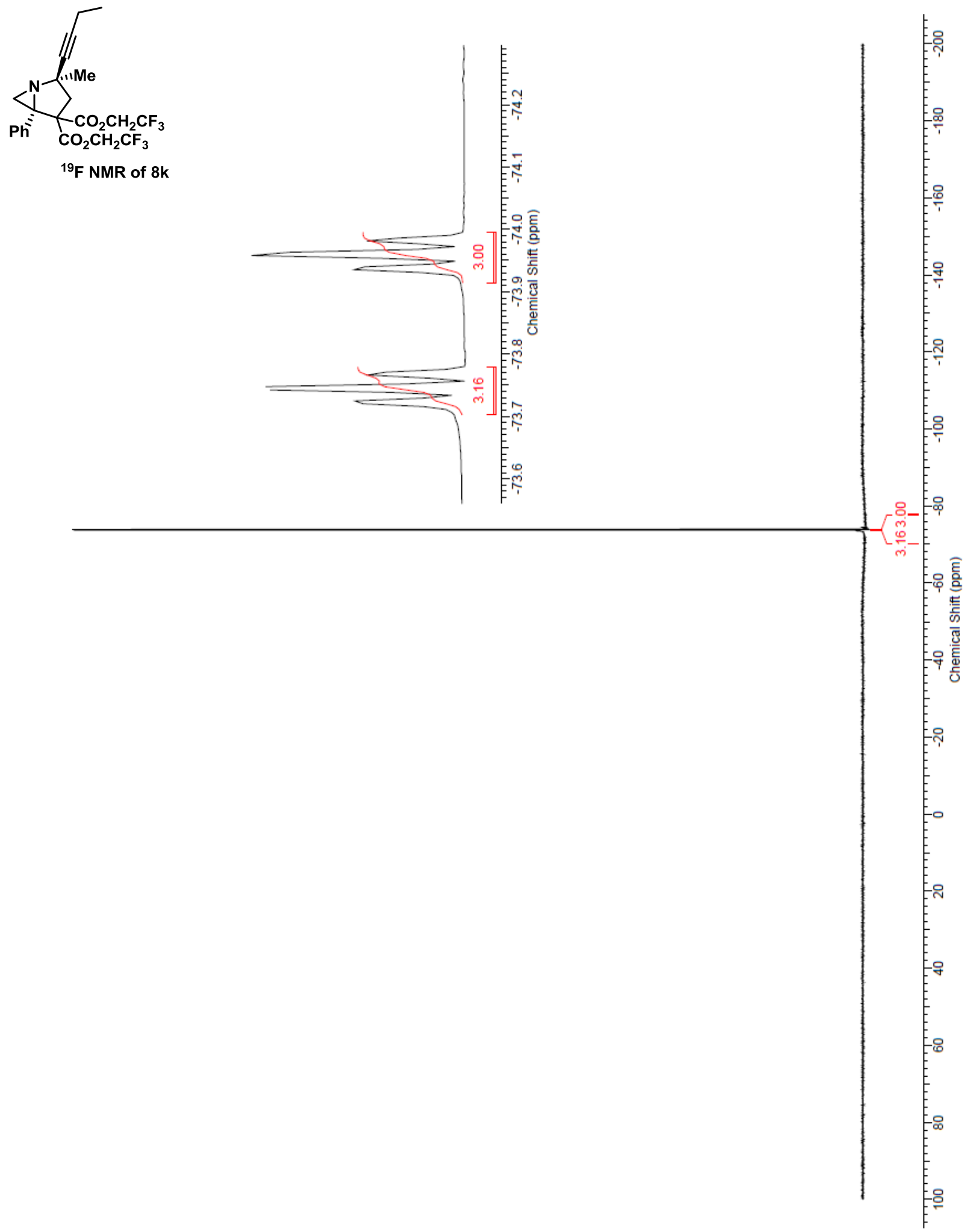


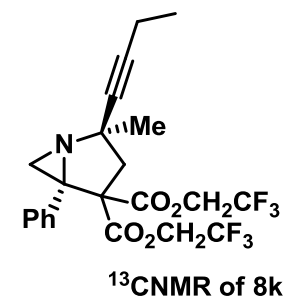

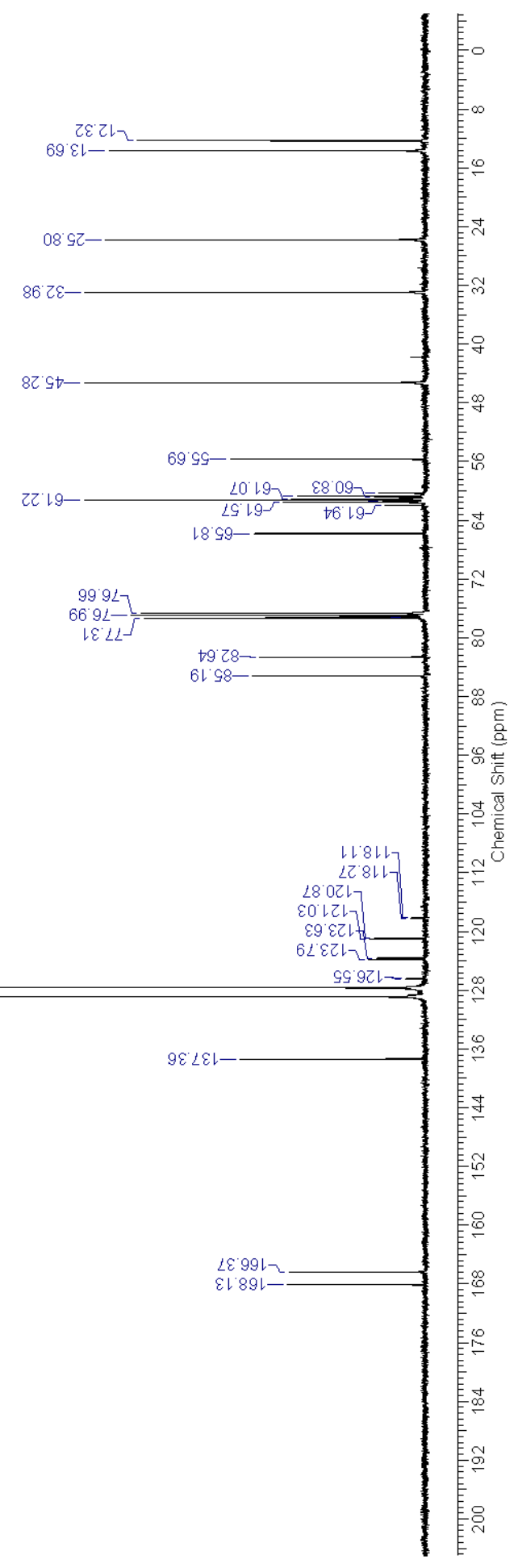

